\title{
Radiation conditions for periodic potentials
}

\author{
Dissertation \\ zur Erlangung des mathematisch-naturwissenschaftlichen Doktorgrades \\ "Doctor rerum naturalium" \\ der Georg-August-Universität Göttingen
}

\author{
vorgelegt von \\ Nguyen Thai Ngoc \\ aus Hanoi, Vietnam
}

Göttingen 2017 
Referent: Prof. Dr. Ingo Witt

Korreferent: Prof. Dr. Wolfram Bauer

Tag der mündliche Prüfung: 


\section{Abstract}

The aim of the present thesis is to determine radiation conditions for higher-dimensional Schrödinger equations with periodic potentials. There is well-known result for Helmholtz equations. However to my knowledge, there have been only a few works on periodic Schrödinger equations.

We employ Floquet Bloch theory to study periodic Schrödinger operators. The limiting absorption principal derives integral formulas for solutions of periodic Schrödinger equations. Then, we use the analytic perturbation theory and the stationary phase method to establish the radiation conditions. 


\section{Acknowledgments}

First and foremost, I would like to express my deepest gratitude to my supervisor Prof. Dr. Ingo Witt for his guidance, assistance, encouragement and patient. Around him I have learned many things from his seminars, lectures. I would like to thank my second supervisor Prof. Dr. Wolfram Bauer for his lecture on "Operator algebras and spectral theory". I also would like to thank all members of the working group seminar "Analysis of Partial Differential Equations".

I am grateful to all my colleagues in the Research Training Group 1493 "Mathematical Structures in Modern Quantum Physics" for their scientific support, especially Thang, Huong, Jiguang, Daoyin, Bingbing, Maia, Matthias, Robin, Yingbo, Sulliman, Roy. I am also thankful for the financial support of Lotus II, The Erasmus Mundus Action Two.

I am indebted to the following people for useful discussions: Peter Kuchment, Vu Hoang, Sonia Fliss, Do Thanh Ngoc, Nguyen Van Lien.

Finally, I would like to thank my family and closed friends for their love, encouragement and help during my stay in Göttingen. 


\section{Contents}

Abstract i

Acknowledgments ii

1 Introduction 1

1.1 The Sommerfeld radiation condition for homogeneous media . . . . . . 2

1.2 The limiting absorption principle for periodic media . . . . . . . . 3

1.3 The radiation condition for periodic waveguides . . . . . . . . . 6

1.4 Main results . . . . . . . . . . . . . . . . . . . . 7

1.5 Summary . . . . . . . . . . . . . . . . . . . 10

2 Floquet-Bloch theory for periodic Schrödinger operators $\quad 13$

2.1 Floquet-Bloch-Gelfand transform . . . . . . . . . . . 13

2.2 Spectral theory of periodic Schrödinger operators . . . . . . . . . . 17

2.2.1 Direct integral of periodic Schrödinger operators . . . . . . . . 17

2.2.2 Band gap structure of the spectrum . . . . . . . . . . . 21

2.2 .3 Bloch waves . . . . . . . . . . . . . . . 23

2.2 .4 Dispersion relation . . . . . . . . . . . . . . . . . 24

3 The one-dimensional case $\quad 27$

3.1 Floquet theory for Hill's operator . . . . . . . . . . . . . . . 27

3.1 .1 The discriminant . . . . . . . . . . . . . . . . 27

3.1.2 Band gap structure of the spectrum . . . . . . . . . . 30

3.2 Radiation conditions for Hill's equation . . . . . . . . . . . . . 32

4 The higher-dimensional case $\quad 39$

4.1 The limiting absorption principal for periodic operators . . . . . . . . 39 
4.2 Radiation conditions in higher dimensions . . . . . . . . . . . . 41

$\begin{array}{ll}\text { A Appendix } & 63\end{array}$

A.1 Spectral theory of self-adioint operators . . . . . . . . . . . . 63

A.1.1 Self-adioint operators . . . . . . . . . . . . . . 63

A.1.2 The spectral theorem of self-adjoint operators . . . . . . . . 66

A.2 Analytic perturbation theory . . . . . . . . . . . . . . 73

A.3 The stationary phase method . . . . . . . . . . . . . . . 77

$\begin{array}{lr}\text { Bibliography } & 79\end{array}$ 


\section{Introduction}

The stationary Schrödinger operator $H=-\Delta+V(x)$ on $L^{2}\left(\mathbb{R}^{d}\right)$ with a periodic potential $V$ has been intensively studied for many years by both mathematicians and physicists. It plays an important role in solid state physics and has applications to photonic crystal, metameterial, carbon nanostructure, and topological insulator. The main tool for periodic operators, Floquet-Bloch-Gelfand transform, reduces the study of a periodic operator on whole space $\mathbb{R}^{d}$ to the study of this operator on a bounded domain with various boundary conditions. Perturbation theory exhibits the band gap structure of the spectrum of the operator $H$. The main purpose of this thesis is to study radiation conditions for the corresponding periodic Schrödinger equation

$$
-\Delta u(x)+V(x) u(x)-\lambda u(x)=f(x)
$$

where $V$ is a smooth real-valued function on $\mathbb{R}^{d}$, periodic with respect to the integer lattice $\mathbb{Z}^{d}, f \in C_{\mathrm{c}}^{\infty}\left(\mathbb{R}^{d}\right)$ and $\lambda$ is the spectral parameter in the spectrum of $H$. Radiation conditions model wave propagation in periodic media when the frequency is in the spectrum.

The radiation conditions in homogeneous media are named after Sommerfeld [Som10, Som12]. These conditions ensure the unique solvability of the Helmholtz equation. We are interested in providing such conditions at infinity and prove the unique solvability of the corresponding boundary value problem of (1.1). The outgoing (physical) solution is derived by using the limiting absorption principle, i.e., one adds a small absorption $i \epsilon, \epsilon \neq 0$ to the spectral parameter and get the unique $L^{2}$-solution of the equation

$$
-\Delta u_{\epsilon}(x)+V(x) u_{\epsilon}(x)-(\lambda+i \epsilon) u_{\epsilon}(x)=f(x)
$$

The family of solutions $\left(u_{\epsilon}\right)_{\epsilon>0}$ converges to a solution $u$ of 11.1 as $\epsilon \rightarrow+0$ in a suitable sense. This limit is called the outgoing or physical solution for the periodic Schrödinger equation without absorption. The precise expression of the solution $u$ allows to analyze 
the asymptotics at infinity and give an intrinsic characterization of the outgoing solution. More generally, the result could be extended to linear second-order elliptic equations on $\mathbb{R}^{d}$ with periodic coefficients and systems. Let us first look at known results:

\subsection{The Sommerfeld radiation condition for homogeneous media}

In 1912, Sommerfeld introduced boundary conditions at infinity which ensure the unique solvability of certain boundary value problems [Sch92]. He considered three dimensional Helmholtz equation in exterior domain [Som10, Som12]

$$
\left\{\begin{aligned}
\Delta u+k^{2} u=0 & \text { in } \quad \mathbb{R}^{3} \backslash K, \\
u=u_{0} & \text { on } \quad \partial K,
\end{aligned}\right.
$$

where $k>0, K$ is a compact set in $\mathbb{R}^{3}$ and it is assumed that $\mathbb{R}^{3} \backslash K$ is connected. The Helmholtz equation arises when one considers time harmonic wave equations. Because the domain $\mathbb{R}^{3} \backslash K$ is unbounded, it implies that $k^{2}$ is in the interior of the spectrum of Laplace operator $-\Delta$ on $L^{2}\left(\mathbb{R}^{3} \backslash K\right)$. Without any further conditions, the Helmholtz equation (1.2) is not uniquely solvable. Sommerfeld had found precise boundary conditions at infinity that ensure the unique solvability of the Helmholtz equation (1.2)

$$
u=O\left(\frac{1}{r}\right), \quad \frac{\partial u}{\partial r}-i k u=o\left(\frac{1}{r}\right) \quad \text { as } \quad r \rightarrow \infty,
$$

where $r=\sqrt{x^{2}+y^{2}+z^{2}}$. The conditions (1.3) are called outgoing radiation conditions which have physical meaning, i.e., waves propagate to infinity. Later on, Rellich strengthened the result by removing the first condition in (1.3) and derived a radiation condition for arbitrary dimension $d$ [Rel43]

$$
r^{(d-1) / 2}\left(\frac{\partial u}{\partial r}-i k u\right)=o(1) \quad \text { as } \quad r \rightarrow \infty .
$$

Remark 1.1. The case $k=0$ can be done by a special treatment (see [Tay11, Section 9.1]). Conditions which ensure the existence and uniqueness are

$$
u(x)=O\left(\frac{1}{r}\right), \quad \partial_{r} u(x)=o\left(\frac{1}{r}\right) \quad \text { as } \quad r \rightarrow \infty .
$$




\subsection{The limiting absorption principle for periodic media}

There are many approaches to the limiting absorption principle. The classical result was found by D.M. Ėŭd [Ëĭd69]. He established the limiting absorption for a class of elliptic operators

$$
H=-\sum_{k, l}^{d} \frac{\partial}{\partial x_{k}}\left(a_{k l}(x) \frac{\partial}{\partial x_{l}}\right)+V(x) \quad \text { on } \quad L^{2}\left(\mathbb{R}^{d}\right),
$$

where $a_{k l}(x)=\delta_{k l}\left(\delta_{k l}\right.$ is the Kronecker) outside of some sphere and $V(x)$ decays sufficiently fast at infinity. He used the Green function for $-\Delta$ and some estimates in weighted Sobolev spaces to derive the limiting absorption principle for operator $H$ with $f(x)$ a sufficiently decaying function, $\lambda$ in the interior of the spectrum $\sigma(H)$. He also got a radiation condition for three dimensional case

$$
\lim _{r \rightarrow \infty} \int_{S(0, r)}\left|\frac{\partial u}{\partial r}-i k u\right|^{2} d \sigma=0,
$$

where $S(0, r)$ is the sphere of radius $r$.

Recently, in [BA11] the author made use of the regularity of the projection-valued measure associated with a self-adjoint operator $H$ on a Hilbert space $\mathscr{H}$ to derive the limiting absorption principle. The resolvent operator $R(z)=(H-z)^{-1}$ is defined in the upper (lower) half plane $\mathbb{C}^{ \pm}=\{z: \pm \operatorname{Im} z>0\}$.

Then, it is clear that $R(z)$ is not convergent in the norm topology of $\mathscr{L}(\mathscr{H})$, the space of linear bounded operators from $\mathscr{H}$ to $\mathscr{H}$, when $z \rightarrow \lambda$ for $\lambda \in \sigma(H)$. But the convergence can be achieved in a weaker topology.

Definition 1.2 ([BA11, Definition 3.1]). Let $\mathscr{H}$ be a separable Hilbert space and $\mathscr{X}$ be another Hilbert space such that the embedding $\mathscr{X} \hookrightarrow \mathscr{H}$ is continuous, $\mathscr{X}^{*}$ is anti-dual of $\mathscr{X}$. A self-adjoint operator $H$ on $\mathscr{H}$ satisfies the limiting absorption principle in an open set $U \subset \mathbb{R}$ if the limits

$$
R^{ \pm}(\lambda)=\lim _{\varepsilon \rightarrow+0} R(\lambda \pm i \varepsilon), \quad \forall \lambda \in U
$$

exit in the norm topology of $\mathscr{L}\left(\mathscr{X}, \mathscr{X}^{*}\right)$.

Ben-Artzi derived a general criteria for obtaining the limiting absorption principle:

Definition 1.3 ([BA11, Definition 3.3]). Let $E(\lambda)$ be the projection-valued measure associated with a self-adjoint operator $H, \mathscr{X}_{H}^{*}$ be another Hilbert space so that the embed$\operatorname{ding} \mathscr{X}_{H}^{*} \hookrightarrow \mathscr{X}$ is continuous. Assume $U \subset \mathbb{R}$ is open and $0<\alpha \leq 1$. Then, $H$ is of the type $\left(\mathscr{X}, \mathscr{X}_{H}^{*}, \alpha, U\right)$ if 
i) $\lambda \mapsto E(\lambda) \in \mathscr{L}\left(\mathscr{X}, \mathscr{X}^{*}\right), \lambda \in U$ is weakly differentiable with a locally Hölder continuous derivative in $\mathscr{L}\left(\mathscr{X}, \mathscr{X}_{H}^{*}\right)$, i.e.,

$$
\frac{d}{d \lambda}(E(\lambda) x, y)=\langle A(\lambda) x, y\rangle \quad \text { for all } x, y \in \mathscr{X}, \lambda \in U,
$$

where $A(\lambda) \in \mathscr{L}\left(\mathscr{X}, \mathscr{X}_{H}^{*}\right)$ is locally Hölder continuous.

ii) For every bounded open set $J \subset U$ and a compact interval $K \subset J$, the operatorvalued function

$$
z \mapsto \int_{U \backslash J} \frac{A(\lambda)}{\lambda-z} d \lambda, \quad z \in \mathbb{C}, \operatorname{Re} z \in K,|\operatorname{Im} z| \leq 1
$$

is Hölder continuous in $\mathscr{L}\left(\mathscr{X}, \mathscr{X}_{H}^{*}\right)$ with exponent $\alpha$.

Theorem 1.4 ([BA11, Theorem 3.6]). Let $H$ be of the type $\left(\mathscr{X}, \mathscr{X}_{H}^{*}, \alpha, U\right)$, where $U \subset \mathbb{R}$ is open and $0<\alpha \leq 1$. Then, $H$ satisfies the limiting absorption principle in $U$. More precisely, the limits

$$
R^{ \pm}(\lambda)=\lim _{\varepsilon \rightarrow+0} R(\lambda \pm i \varepsilon)
$$

exit in the norm topology of $\mathscr{L}\left(\mathscr{X}, \mathscr{X}_{H}^{*}\right)$.

In [BY94], the authors proved that in a certain range of the spectrum the derivative of the projection-valued measure associated with $H$ is Höder continuous. This is fulfilled for $\lambda$ close enough to the bottom of the spectrum of a periodic Schrödinger operator. As a consequence of Theorem 1.4, the limiting absorption principle holds for a small interval near the bottom of the spectrum of the periodic Schrödinger operator. This method can not be extended to large $\lambda$ because the Hölder continuity of the projectionvalued measure is not satisfied in general.

The limiting absorption principle for a large class of periodic operators and a large range of the spectral parameter was extended by Maria Radosz [ $[\operatorname{Rad} 15]$. The author regarded the solution as a distribution of both $\lambda$ and variable $x$. In her thesis $[\operatorname{Rad} 10]$, she considered the equation

$$
H u-\lambda u=f
$$

where operator $H$ is elliptic and periodic with respect to some lattice $\Gamma$ and satisfies some properties for band functions and Bloch waves. More precisely, the periodic operator $H$ 
can be written as a direct integral

$$
H=\int_{B}^{\oplus} H(k) d k,
$$

where $B$ is the Brillouin zone for the periodic lattice $\Gamma$, and $H(k)$ has the same formula as $H$ but acts on the space $L^{2}(W)$ ( $W$ is the fundamental region for $\Gamma$ ) with quasi-periodic boundary conditions.

The operator $H(k)$ has compact resolvent. Thus, it has the discrete spectrum enumerated with multiplicities

$$
\lambda_{1}(k) \leq \lambda_{2}(k) \leq \cdots \leq \lambda_{j}(k) \leq \cdots \rightarrow \infty
$$

Functions $\lambda_{j}(k), j \in \mathbb{N}$ are continuous and called the band functions. The corresponding eigenfunctions $\psi_{j}(x, k), j \in \mathbb{N}$ are called Bloch waves. These eigenfunctions can be chosen as measurable functions in $k$.

Definition 1.5 ([ Rad10, Definition 2.1], distributional solution). A distribution $u$ acting on test functions $\phi(\lambda, x) \in C_{\mathrm{c}}^{\infty}\left(\mathbb{R} \times \mathbb{R}^{d}\right)$ is called a distribution solution of (1.5) if it is locally integrable in $\mathbb{R} \times \mathbb{R}^{d}$ and for all $\phi \in C_{\mathrm{c}}^{\infty}\left(\mathbb{R} \times \mathbb{R}^{d}\right)$

$$
u[(H-\lambda) \phi]=\int_{\mathbb{R}} \int_{\mathbb{R}^{d}} f(\lambda, x) \phi(\lambda, x) d x d \lambda
$$

Definition 1.6 $\left(\left[\operatorname{Rad} 15\right.\right.$, Definition 2.1]). $\quad$ i) $\lambda \in \mathbb{R}$ is called a regular value of $\lambda_{j}, j \in$ $\mathbb{N}$ if for every $k$ such that $\lambda_{j}(k)=\lambda$, then $\nabla \lambda_{j}(k) \neq 0$. A value that is not regular is called singular.

ii) The set of regular values is defined by

$$
\mathcal{R}=\left\{\lambda \in \mathbb{R}: \lambda \text { is a regular value of } \lambda_{j} \text { for all } j \in \mathbb{N}\right\}
$$

Theorem 1.7 ( $\operatorname{Rad15}$, Theorem 1.2]). For $f \in L^{2}\left(\mathbb{R}^{d}\right)$ and $\lambda$ is regular, the limit

$$
u(\lambda, x):=\lim _{\varepsilon \rightarrow+0} u_{\epsilon}(\lambda, x)=\lim _{\varepsilon \rightarrow+0}(H-\lambda-i \varepsilon)^{-1} f
$$

exists in $L_{\mathrm{loc}}^{2}\left(\mathcal{R} ; L^{2}\left(\mathbb{R}^{d}, \omega(x) d x\right)\right)$ for some weighted function $\omega(x)$.

She also obtained an explicit integral formula for the distribution $u(\lambda, x)$ by using the Floquet-Bloch-Gelfand transform.

The limiting absorption principle for periodic operators and the Sommerfeld radiation conditions motivate to find similar radiation conditions for periodic Schrödinger equations. Such conditions could have applications in solid state physics and photonic crytal. 


\subsection{The radiation condition for periodic waveguides}

Periodic waveguide is a cylinder which is periodic in one direction and bounded in other directions. In [Hoa11], the author contributed to semi-infinite waveguide problem which is of the form

$$
\left\{\begin{array}{lc}
(H-\lambda g) u=0 & \text { in } W^{+}, \\
u=\varphi \in H^{\frac{1}{2}}(\Gamma) \quad \text { on } \Gamma, \quad \frac{\partial u}{\partial v}=0 & \text { on } \partial W^{+} \backslash \Gamma,
\end{array}\right.
$$

where $H$ is a symmetric second-order elliptic operator with periodic coefficients, $W^{+}=$ $(0, \infty) \times(0,1), \Gamma=\{0\} \times(0,1), g(x, y) \in L^{\infty}\left(\mathbb{R}_{+} \times(0,1)\right)$ bounded from below by a positive constant and periodic in $x$ direction with the same period. By adding a small absorption $i \epsilon, \epsilon>0$ to $\lambda$, there exists a unique $L^{2}$-solution $u_{\epsilon}$ of the equation with absorption. He obtained the limiting absorption principle:

Theorem 1.8 ([Hoa11, Theorem 1.1]). For any regular value $\lambda_{0}$ in the sense of Definition 1.6 there exist a neighborhood $\mathcal{N} \subset \mathbb{R}$ of $\lambda_{0}$ and a finite subset $\Sigma$ of $\mathcal{N}$ such that for all $\lambda \in \mathcal{N} \backslash \Sigma$ the solution $u_{\epsilon} \rightarrow$ u as $\epsilon \rightarrow+0$ in $H_{\text {loc }}^{1}\left(W^{+}\right)$.

The limit solution $u$ has a nice property. It is a finite linear combination of the Bloch waves plus a decaying function. He also proved this property is a radiation condition which ensures the unique solvability.

Theorem 1.9 ([Hoa15, Theorem 1.1]). Let $\lambda$ be regular, and $Z_{\lambda}$ be a space of all functions $u_{0}+u_{1}$, where $u_{0} \in H^{1}\left(W^{+}\right)$and $u_{1}$ is a finite linear combination of outgoing Bloch waves. Then, for $\lambda \in \mathcal{N} \backslash \Sigma$, (1.6) has a unique solution $u \in Z_{\lambda}$.

In [FJ16], the authors extended this result for periodic waveguide. Namely, they considered the equation

$$
\left\{\begin{array}{l}
\Delta u-\lambda g u=f \text { in } \Omega \\
\frac{\partial u}{\partial v}=0 \text { on } \partial \Omega,
\end{array}\right.
$$

where $\Omega$ is a connected open set in $\mathbb{R}^{1+d}$ which is periodic with period $L$ in the first variable and bounded in the others, i.e., $\left(x_{1}, x_{s}\right) \in \Omega \Rightarrow\left(x_{1}+L, x_{s}\right) \in \Omega$. The function $g \in L^{\infty}$ is bounded from below by a positive constant and periodic with the same period as the waveguide in the first variable, i.e., $g\left(x_{1}+L, x_{s}\right)=g\left(x_{1}, x_{s}\right)$ for all $\left(x_{1}, x_{s}\right) \in \Omega$.

If the right hand side $f \in L^{2}(\Omega)$ has compact support, then the limiting absorption principle holds for the regular value $\lambda$ and the radiation condition is similar to the case of 
semi-infinite waveguide, i.e., the unique solution can be characterized as a finite linear combination of Bloch waves plus a decreasing function.

The above results are done only for domains periodic in one direction and bounded in others. This motivates to study radiation conditions for periodic Schödinger equations in higher dimensions. Many materials have structure of periodicity in two or higher dimensions, and such results will have applications in wave propagation in periodic background.

\subsection{Main results}

The radiation conditions obtained in [Hoa15] for periodic semi-infinite waveguide and in [FJ16] for periodic waveguide can be extended for perfect periodic media which is periodic in more than one direction. The proof in [Hoa15, FJ16] deeply relies on the analytic perturbation theory [Re143, Kat95] to get analyticity of the band functions and Bloch waves and uses methods from complex analysis to analyze solutions. The cases of periodicity in more than one direction, the analyticity is not true in general. In order to overcome the difficulty, we firstly localize the integral representation of the solution near the level set. Then, we assume some conditions on the band functions and Bloch waves near the level set and use the stationary phase method to get an asymptotic expansion of the solution.

In the thesis [Fli09], the author put a conjecture [Fli09, Conjecture 4.4.17] about the asymptotic expansion of the outgoing solution in two dimension. She did not give any further condition. The main result of this thesis is to generalize this asymptotic expansion to higher dimensions and prove the unique solvability of the corresponding equation under the radiation conditions.

In [MT06], authors considered a second-order elliptic operator with periodic coefficients

$$
H=-\sum_{k, l=1}^{d} \frac{\partial}{\partial x_{k}}\left(a_{k l}(x) \frac{\partial}{\partial x_{l}}\right)+b(x) \quad \text { on } \quad L^{2}\left(\mathbb{R}^{d}\right),
$$

where $d \geq 2$, the coefficients are real-valued measurable and periodic with respect to $\mathbb{Z}^{d}$. Furthermore, the matrix $a=\left(a_{k l}(x)\right)_{k, l=1}^{d}$ is symmetric satisfying

$$
c^{-1}|\xi|^{2} \leq \sum_{k, l=1}^{d} a_{k l}(x) \xi_{k} \xi_{l} \leq c|\xi|^{2}
$$


for some $c>0$.

They derived an asymptotics of the Green function for operator $(H-\lambda-i 0)=$ $\lim _{\epsilon \rightarrow+0}(H-\lambda-i \epsilon)^{-1}$ with spectral parameter close and greater than the bottom of the spectrum of operator $H$. Later on, in [KR12, KKR17] the authors extended the result to the case spectral parameter in a gap near the edges and at edges of the spectrum for generic periodic elliptic operators of second-order. Such asymptotics of the Green functions can be used to characterize the solution of the corresponding equation.

We are interested in the stationary Schrödinger equation

$$
-\Delta u+V(x) u-\lambda u=f(x), \quad x \in \mathbb{R}^{d},
$$

where $V$ is a smooth real-valued function on $\mathbb{R}^{d}$, periodic with respect to the integer lattice $\mathbb{Z}^{d}, f \in C_{\mathrm{c}}^{\infty}\left(\mathbb{R}^{d}\right), \lambda \in \mathbb{R}$ is the spectral parameter in the spectrum. Our results could be generalized to periodic elliptic operators of second-order with periodic coefficients.

The corresponding operator $H=-\Delta+V$ on $L^{2}\left(\mathbb{R}^{d}\right)$ is self-adjoint and has the band gap spectral [Kuc93]. Namely, there are sequences of the band functions $\lambda_{j}(k)$ and the corresponding Bloch waves $\psi_{j}(x, k), j \in \mathbb{N}$. To formulate the main results, define a set

$$
J(\lambda)=\left\{j \in \mathbb{N}: \exists k \in B, \lambda_{j}(k)=\lambda\right\},
$$

and the level sets of $\lambda$

$$
\Gamma_{j}(\lambda)=\left\{k \in[-\pi, \pi]^{d}: \lambda_{j}(k)=\lambda\right\} \quad \text { for } j \in J(\lambda) .
$$

For $\omega \in \mathbb{S}^{d-1}$, define

$$
L_{j}(\omega)=\left\{k_{\omega} \in \Gamma_{j}(\lambda): \omega=\nabla \lambda_{j}\left(k_{\omega}\right) /\left|\nabla \lambda_{j}\left(k_{\omega}\right)\right|\right\} \quad \text { for } j \in J(\lambda)
$$

The first main result in this thesis is to characterize the outgoing (physical) solution of (1.7) obtained by the limiting absorption principle in the distributional sense $[\operatorname{Rad10}, \operatorname{Rad15}]$. The distribution solution for regular spectral parameter is adapted to periodic Schrödinger equation with smooth coefficients by using elliptic regularity [RS75, Fo195]. The Floquet-Bloch theory [Kuc93, RS78, Plu11] gives the integral representation of the limiting solution. The analytic perturbation theory allows to localize the integral representation of the solution near the level sets of $\lambda$. The stationary phase method provides the asymptotic expansion of the solution. The assumption used in the proof is the local analyticity of the band functions and Bloch waves near the level sets. 
Theorem 1.10 (Outgoing radiation condition in higher dimensions). Let $\lambda \in \mathbb{R}$ be regular. Assume that there exists a system of locally smooth band functions $\lambda_{j}(k)$ and Bloch waves $\psi_{j}(k, x)$ near level sets $\Gamma_{j}(\lambda), j \in J(\lambda)$; the number of points in $L_{j}(\omega)$ is finite for all $j \in J(\lambda), \omega \in \mathbb{S}^{d-1}$, and Gauss-Kronecker curvature of $\Gamma_{j}(\lambda)$ oriented by $-\nabla \lambda_{j}(k) /\left|\nabla \lambda_{j}(k)\right|$ at any point $k_{\omega} \in L_{j}(\omega), j \in J(\lambda)$ is non-zero. Then, there is an asymptotic expansion of solution $u(x)=\lim _{\epsilon \rightarrow+0} u_{\epsilon}(x)$ in $L_{\text {loc }}^{2}\left(\mathbb{R}^{d}\right)$ of 1.7

$$
\begin{aligned}
u(x+n)=\frac{i}{(2 \pi)^{(d-1) / 2}} \sum_{j \in J(\lambda)} \sum_{k_{\omega} \in L_{j}(\omega)} \frac{A_{j}\left(x, \lambda, k_{\omega}\right)}{|n|^{(d-1) / 2}} e^{i|n| k_{\omega} \cdot \omega}+ & \\
& O\left(\frac{1}{|n|^{(d+1) / 2}}\right) \quad \text { as }|n| \rightarrow \infty,
\end{aligned}
$$

uniformly in $x \in[0,1]^{d}$ and in $\omega=n /|n| \in \mathbb{S}^{d-1}$. Here,

$$
\text { - } A_{j}\left(x, \lambda, k_{\omega}\right)=\frac{\left\langle\mathcal{U} f\left(., k_{\omega}\right), \psi_{j}\left(., k_{\omega}\right)\right\rangle e^{\frac{-i \pi}{4} \operatorname{sgn}\left(e_{l}^{\prime} \cdot \operatorname{Hess} \lambda_{j}\left(k_{\omega}\right) e_{m}^{\prime}\right)_{l, m=2}^{d}}}{\left|\nabla \lambda_{j}\left(k_{\omega}\right)\right| \sqrt{\left|K_{j}\left(k_{\omega}\right)\right|}} \psi_{j}\left(x, k_{\omega}\right),
$$

where $\left(\omega, e_{2}^{\prime}, \ldots, e_{d}^{\prime}\right)$ is an orthonormal basic of $\mathbb{R}^{d}$, and $K_{j}\left(k_{\omega}\right)$ is the GaussKronecker curvature of $\Gamma_{j}(\lambda)$ at $k_{\omega}$.

The second result is to prove the unique solvability under the radiation condition (1.8). This is a direct consequence of spectral theorem.

Theorem 1.11 (Uniqueness for outgoing solution). Assume $\lambda \in \mathbb{R}$ is regular and the assumption in Theorem 1.10 satisfies. Then, there is a unique solution to $-\Delta u+V(x) u-$ $\lambda u=f(x)$ satisfying the asymptotic expansion

$$
u(x+n)=\frac{i}{(2 \pi)^{(d-1) / 2}} \sum_{j \in J(\lambda)} \sum_{k_{\omega} \in L_{j}(\omega)} \frac{A_{j}\left(x, \lambda, k_{\omega}\right)}{|n|^{(d-1) / 2}} e^{i|n| k_{\omega} \cdot \omega}+O\left(\frac{1}{|n|^{(d+1) / 2}}\right) \quad \text { as }|n| \rightarrow \infty
$$

uniformly in $x \in[0,1]^{d}$ and in $\omega=n /|n| \in \mathbb{S}^{d-1}$.

If we take the limit of $u_{\epsilon}$ as $\epsilon \rightarrow-0$ instead of $\epsilon \rightarrow+0$, then we will get the incoming solution. A incoming radiation condition is similar to the outgoing radiation condition.

Theorem 1.12 (Incoming radiation condition). Let $\lambda \in \mathbb{R}$ be regular. Assume that there exists a system of locally smooth band functions $\lambda_{j}(k)$ and Bloch waves $\psi_{j}(k, x)$ near level sets $\Gamma_{j}(\lambda), j \in J(\lambda)$; the number of points in $L_{j}(-\omega)$ is finite for all $j \in J(\lambda), \omega \in$ $\mathbb{S}^{d-1}$, and Gauss-Kronecker curvature of $\Gamma_{j}(\lambda)$ oriented by $-\nabla \lambda_{j}(k) /\left|\nabla \lambda_{j}(k)\right|$ at any point 
$k_{\omega} \in L_{j}(-\omega), j \in J(\lambda)$ is non-zero. Then, there is an asymptotic expansion of solution $u(x)=\lim _{\epsilon \rightarrow-0} u_{\epsilon}(x)$ in $L_{\mathrm{loc}}^{2}\left(\mathbb{R}^{d}\right)$ of

$$
\begin{aligned}
& u(x+n)=\frac{-i}{(2 \pi)^{(d-1) / 2}} \sum_{j \in J(\lambda)} \sum_{k_{\omega} \in L_{j}(-\omega)} \frac{A_{j}\left(x, \lambda, k_{\omega}\right)}{|n|^{(d-1) / 2}} e^{i n \mid k_{\omega} \cdot \omega}+ \\
& O\left(\frac{1}{|n|^{(d+1) / 2}}\right) \quad \text { as }|n| \rightarrow \infty,
\end{aligned}
$$

uniformly in $x \in[0,1]^{d}$ and in $\omega=n /|n| \in \mathbb{S}^{d-1}$. Here,

- $L_{j}(-\omega)=\left\{k_{\omega} \in \Gamma_{j}(\lambda): \omega=-\nabla \lambda_{j}\left(k_{\omega}\right) /\left|\nabla \lambda_{j}\left(k_{\omega}\right)\right|\right\} \quad$ for $\quad j \in J(\lambda)$,

- $A_{j}\left(x, \lambda, k_{\omega}\right)=\frac{\left\langle\mathcal{U} f\left(., k_{\omega}\right), \psi_{j}\left(., k_{\omega}\right)\right\rangle e^{\frac{i \pi}{4} \operatorname{sgn}\left(e_{l}^{\prime} \cdot \operatorname{Hess} \lambda_{j}\left(k_{\omega}\right) e_{m}^{\prime}\right)_{l, m=2}^{d}}}{\left|\nabla \lambda_{j}\left(k_{\omega}\right)\right| \sqrt{\left|K_{j}\left(k_{\omega}\right)\right|}} \psi_{j}\left(x, k_{\omega}\right)$,

where $\left(\omega, e_{2}^{\prime}, \ldots, e_{d}^{\prime}\right)$ is an orthonormal basic of $\mathbb{R}^{d}$, and $K_{j}\left(k_{\omega}\right)$ is the GaussKronecker curvature of $\Gamma_{j}(\lambda)$ at $k_{\omega}$.

We also obtain unique solvability under the incoming radiation condition (1.9).

Theorem 1.13 (Uniqueness for incoming solution). Assume $\lambda \in \mathbb{R}$ is regular and the assumption in Theorem 1.12 satisfies. Then, there is a unique solution to $-\Delta u+V(x) u-$ $\lambda u=f(x)$ satisfying the asymptotic expansion

$u(x+n)=\frac{-i}{(2 \pi)^{(d-1) / 2}} \sum_{j \in J(\lambda)} \sum_{k_{\omega} \in L_{j}(-\omega)} \frac{A_{j}\left(x, \lambda, k_{\omega}\right)}{|n|^{(d-1) / 2}} \exp \left(i|n| k_{\omega} \cdot \omega\right)+O\left(\frac{1}{|n|^{(d+1) / 2}}\right) \quad$ as $|n| \rightarrow \infty$, uniform in $x \in[0,1]^{d}$ and in $\omega=n /|n| \in \mathbb{S}^{d-1}$.

\subsection{Summary}

My thesis contains five chapters:

1. In Chapter 1, we give an introduction and summary of results.

2. In Chapter 2, we recall Floquet-Bloch theory for periodic Schrödinger operators, including the Floquet-Bloch-Gelfand transform, the direct integral, the band gap structure of the spectrum, and Bloch waves. These are essential to study the limiting absorption principle and radiation conditions for periodic equations. 
3. Chapter 3 studies radiation conditions in one-dimensional case. The Floquet theory is presented for Hill's operator to derive band gap structure of spectrum. Then, using Sturm-Liouville theory, we obtain nice radiation conditions.

4. Chapter 4 is devoted to study radiation conditions in higher-dimensional cases. We recall the limiting absorption principle for distributional solution $\llbracket \operatorname{Rad} 10$, Rad15]. Then, using analytic perturbation theory and the stationary phase method, we will get radiation conditions for periodic Schrödinger equation. The unique solvability is obtained under the radiation conditions by using spectral theorem.

5. The thesis ends with an Appendix, where we recall spectral theory of self-adjoint operators, analytic perturbation theory and the stationary phase method. 



\section{Floquet-Bloch theory for periodic Schrödinger operators}

In this chapter, we are going to recall Floquet-Bloch theory for periodic Schrödinger operator $H=-\Delta+V(x)$ [MW66, Eas73, Kuc93, Kuc16, RS78, BES13]. This is one of the most important operators in solid state physics. Section 2.1 is devoted to the Floquet-Bloch-Gelfand transform which is the main analytic tool for periodic operators. In Section 2.2, the direct integral reduces the study of the spectrum of a periodic Schödinger operator on $\mathbb{R}^{d}$ to the discrete spectrum of the same operator on the fundamental domain with various boundary conditions. Then, the analytic perturbation theory gives the band gap structure of the spectrum. The notion of Bloch waves, the eigenfunctions corresponding to discrete eigenvalues, are also introduced.

\subsection{Floquet-Bloch-Gelfand transform}

First, we recall some basic definitions concerning the lattice of periodic Schrödinger operators. We follow [Kuc16].

Definition 2.1. A lattice $\Gamma$ in $\mathbb{R}^{d}$ is the set of all integer linear combinations of $d$ linearly independent vectors $a_{1}, a_{2}, \ldots, a_{d} \in \mathbb{R}^{d}$

$$
\Gamma=\left\{\sum_{j=1}^{d} m_{j} a_{j}: m_{j} \in \mathbb{Z}, j=1, \ldots, d\right\}=\mathbb{Z} a_{1} \oplus \mathbb{Z} a_{2} \oplus \cdots \oplus \mathbb{Z} a_{d} .
$$

Definition 2.2. The Wigner-Seitz cell, a fundamental region $W$ of the lattice $\Gamma$, is defined by

$$
W=\left\{\sum_{j=1}^{d} t_{j} a_{j}: 0 \leq t_{j} \leq 1, j=1,2, \ldots, d\right\} .
$$


Definition 2.3. $\quad$ i) The dual lattice is defined by

$$
\Gamma^{*}=\left\{\sum_{j=1}^{d} m_{j} k_{j}: m_{j} \in \mathbb{Z}, j=1, \ldots, d\right\}=\mathbb{Z} k_{1} \oplus \mathbb{Z} k_{2} \oplus \cdots \oplus \mathbb{Z} k_{d},
$$

where $k_{j}, j=1, \ldots, d$ are the dual lattice vectors satisfying the relation

$$
k_{j} \cdot a_{l}=2 \pi \delta_{j l} \quad \text { for all } j, l=1, \ldots, d .
$$

ii) The first Brillouin zone is a fundamental region of the dual lattice $\Gamma^{*}$ defined by

$$
B=\left\{k \in\left(\mathbb{R}^{d}\right)^{*}:|k| \leq|k-b| \text { for any } b \in \Gamma^{*}\right\} .
$$

It is closure of the set of all points which are closer to the origin than any lattice points of the dual lattice $\Gamma^{*}$.

We also introduce two tori that correspond to the two lattices

$$
\mathbb{T}=\mathbb{R}^{d} / \Gamma \text { and } \mathbb{T}^{*}=\mathbb{R}^{d} / \Gamma^{*}
$$

Example 2.4 (Integer lattice). The typical example of lattices is the integer lattice $\mathbb{Z}^{d}$. In this case,

- the fundamental region is $W=[0,1]^{d}$,

- the dual lattice is $\Gamma^{*}=2 \pi \mathbb{Z}^{d}$,

- the Brillouin zone is $B=[-\pi, \pi]^{d}$.

In this thesis, we only consider periodic Schrödinger operators with respect to the integer lattice. But all results hold for any lattice.

Example 2.5 (Honeycomb lattice). Consider a lattice in $\mathbb{R}^{2}$

$$
\Gamma=\mathbb{Z} a_{1} \oplus \mathbb{Z} a_{2},
$$

where $a_{1}=(\sqrt{3} / 2,1 / 2), a_{2}=(\sqrt{3} / 2,-1 / 2)$.

The dual lattice

$$
\Gamma^{*}=\mathbb{Z} k_{1} \oplus \mathbb{Z} k_{2},
$$

where $k_{1}=\frac{4 \pi}{\sqrt{3}}(1 / 2, \sqrt{3} / 2), k_{2}=\frac{4 \pi}{\sqrt{3}}(1 / 2,-\sqrt{3} / 2)$.

The Brillouin zone is a hexagon in $\mathbb{R}^{2}$ which is invariant under a rotation $2 \pi / 3$ about the origin. 
Remark 2.6. The idea for Floquet-Bloch-Gelfand transform comes from Fourier series which identifies $L^{2}(\mathbb{T})$ with the $l^{2}$ space on $\Gamma^{*}$ by

$$
f(x) \mapsto\left\{f_{k}=\int_{\mathbb{T}} f(x) e^{-i k \cdot x} d x\right\}_{k \in \Gamma^{*}} .
$$

The coefficients $f_{k}$ are called Fourier coefficients.

The Floquet-Bloch-Gelfand transform is firstly defined for $f \in \mathscr{S}\left(\mathbb{R}^{d}\right)$, and then extended to $L^{2}\left(\mathbb{R}^{d}\right)$ by continuity.

Definition 2.7 («uc16, Definition 4.1]). For $f \in \mathscr{S}\left(\mathbb{R}^{d}\right)$, Floquet-Bloch-Gelfand transform $\mathcal{U} f$ is defined by

$$
\mathcal{U} f(x, k)=\sum_{\gamma \in \Gamma} e^{i k \cdot \gamma} f(x-\gamma), \quad(x, k) \in \mathbb{R}^{d} \times \mathbb{R}^{d} .
$$

Remark 2.8. A function on the torus $\mathbb{T}\left(\mathbb{T}^{*}\right)$ can be identified with a periodic function on $\mathbb{R}^{d}$ with respect to the lattice $\Gamma\left(\Gamma^{*}\right)$.

Using the density of $\mathscr{S}\left(\mathbb{R}^{d}\right)$ in $L^{2}\left(\mathbb{R}^{d}\right)$, extend $\mathcal{U}$ to $L^{2}\left(\mathbb{R}^{d}\right)$ by continuity.

Theorem 2.9 ([Kuc16, Theorem 4.2]). The map $\mathcal{U}$ is extended to a linear bounded operator

$$
\mathcal{U}: L^{2}\left(\mathbb{R}^{d}\right) \rightarrow L^{2}\left(\mathbb{T}^{*} ; L^{2}(W)\right) .
$$

Moreover, $\|\mathcal{U}\|=1$ if the measure on $B$ and $W$ are normalized to to have total mass 1 . And $\mathcal{U}$ is invertible with $\mathcal{U}^{-1}=\mathcal{U}^{*}$.

Proposition 2.10 ([Kuc16, Section 4]). For $f \in L^{2}\left(\mathbb{R}^{d}\right)$,

i)

$$
\mathcal{U} f\left(x, k+k^{*}\right)=\mathcal{U} f(x, k), \quad k^{*} \in \Gamma^{*},
$$

ii)

$$
\mathcal{U} f(x+\gamma, k)=e^{i k \cdot \gamma} \mathcal{U} f(x, k), \quad \gamma \in \Gamma
$$

Remark 2.11. The first property (2.1) shows that $\mathcal{U} f(x, k)$ is periodic in $k$ with respect to the dual lattice $\Gamma^{*}$. Property (2.2) is called quasi-periodic, cyclic or Floquet property of $\mathcal{U} f(x, k)$ with quasi-momentum $k$.

In Theorem 2.9, $\mathcal{U} f(x, \cdot)$ is defined for $x \in W$ satisfying quasi-periodic condition (2.2). If we extend $\mathcal{U} f(x, \cdot)$ from $W$ to all $\mathbb{R}^{d}$ satisfying (2.2), then we get the inverse of Floquet-Bloch-Gelfand transform. 
Theorem 2.12 ([Kuc16, Section 4]). The inverse of the Floquet-Bloch-Gelfand transform has the formulas

$$
\begin{aligned}
& f(x)=\frac{1}{(2 \pi)^{d}} \int_{B} e^{-i k \cdot \gamma} \mathcal{U} f(x+\gamma, k) d k, \quad \gamma \in \Gamma, x \in W-\gamma, \\
& f(x)=\frac{1}{(2 \pi)^{d}} \int_{B} \mathcal{U} f(x, k) d k, \quad x \in \mathbb{R}^{d} .
\end{aligned}
$$

The next theorem shows the relation between the decay of a function and the smoothness of its Floquet-Bloch-Gelfand transform.

Theorem 2.13 ([Kuc16, Theorem 4.2]). Let $g(x, k)$ be $\Gamma^{*}$-periodic in $k$ and quasiperiodic in $x$ with quasi-momentum $k$. Then:

i) If $g(x, k)$ is a smooth function in $k \in \mathbb{R}^{d}$ with values in $L^{2}(W)$, then the inverse Floquet-Bloch-Gelfand transform $f(x):=\mathcal{U}^{-1} g \in L^{2}\left(\mathbb{R}^{d}\right)$, and for any $N>0$

$$
\|f\|_{L^{2}(W+\gamma)} \leq C_{N}|\gamma|^{-N}
$$

ii) If $g(x, k)$ is an analytic function in $k \in \mathbb{R}^{d}$ with values in $L^{2}(W)$, then the inverse Floquet-Bloch-Gelfand transform $f(x):=\mathcal{U}^{-1} g \in L^{2}\left(\mathbb{R}^{d}\right)$, and there exists a positive constant $C_{1}, C_{2}$ such that

$$
\|f\|_{L^{2}(W+\gamma)} \leq C_{1} e^{-C_{2}|\gamma|}
$$

Remark 2.14. The converse of Theorem 2.13 is also true, i.e., if the norm $\|f\|_{L^{2}(W+\gamma)}$ decays when $|\gamma| \rightarrow \infty$ faster than any power of $|\gamma|$ (decays exponential fast), then the Floquet-Bloch-Gelfand transform $\mathcal{U} f(x, k)$ is smooth (analytic) in $k$ with values in $L^{2}(W)$.

One usually deals with Sobolev spaces $H^{s}\left(\mathbb{R}^{d}\right), s \in \mathbb{R}$ instead of $L^{2}\left(\mathbb{R}^{d}\right)$. In this case, the unitary property of Floquet-Bloch-Gelfand transform from $H^{s}\left(\mathbb{R}^{d}\right)$ into $L^{2}\left(\mathbb{T}^{*}, H^{s}(W)\right)$ does not hold because surjectivity breaks down. We need a subspace of $H^{s}(W)$ by adding boundary conditions between two adjacent cells.

Definition 2.15 (Kuc16, Definition 4.4]). For any $k \in \mathbb{R}^{d}, s \geq 0$, the space $H_{k}^{s}(W)$ is defined as

$$
H_{k}^{s}(W)=\left\{f_{\left.\right|_{W}}: f \in H_{\mathrm{loc}}^{s}\left(\mathbb{R}^{d}\right) \text { such that } f \text { satisfies }(2.2)\right\} .
$$

Proposition 2.16 ([Kuc16, Proposition 4.6]). Let $s \geq 0$. Then, for any $k$ 
i) $H_{k}^{s}(W)$ is a closed subspace of $H^{s}(W)$,

ii) $H_{k}^{0}(W)=L^{2}(W)$

iii) A function $u$ belongs to $H_{k}^{s}(W)$ if and only if it can be represented as

$$
u(x)=e^{i k \cdot x} v(x)
$$

where $v(x)$ is $\Gamma$-periodic, i.e., $v \in H^{s}(\mathbb{T})$,

iv) $\mathcal{H}^{s}=\bigcup_{k \in \mathbb{R}^{d}} H_{k}^{s}(W)$ is an real analytic Banach vector subbundle of a trivial bundle $\mathbb{R}^{d} \times H^{s}(W)$ over $\mathbb{R}^{d}$.

Theorem 2.17 ( Kuc16, Theorem 4.8]). For $s \geq 0, \mathcal{U}$ is isometric from $H^{s}\left(\mathbb{R}^{d}\right)$ onto the space $L^{2}\left(\mathbb{T}^{*}, \mathcal{H}^{s}\right)$.

\subsection{Spectral theory of periodic Schrödinger operators}

In this section, we recall spectral theory of periodic Schrödinger operators. The direct integral and analytic perturbation theory show the band gap structure of the spectrum. Bloch waves, eigenfunctions corresponding to eigenvalues, and dispersion relation, the important notion in physics, are also introduced. The main references are [RS78, Kuc93, Kuc16].

Consider the stationary Schrödinger operator

$$
H=-\Delta+V(x)
$$

where $V(x) \in L_{\text {loc }}^{2}\left(\mathbb{R}^{d}\right)$ is real-valued, periodic with respect to the lattice $\Gamma$, i.e.,

$$
V(x)=V(x+\gamma), \quad \forall x \in \mathbb{R}^{d}, \gamma \in \Gamma .
$$

\subsubsection{Direct integral of periodic Schrödinger operators}

Definition 2.18 ([RS78]). Let $\mathscr{K}$ be a separable Hilbert space with the inner product $\langle\cdot, \cdot\rangle$ and $(X, \mu)$ a $\sigma$-finite measure space. Then, we call $\mathscr{H} \cong L^{2}(X, d \mu ; \mathscr{K})$ a constant fiber direct integral and write

$$
\mathscr{H}=\int_{X}^{\oplus} \mathscr{K} d \mu(x)
$$


Proposition 2.19 ([RS78]). $\mathscr{H}$ is a Hilbert space with respect to the norm

$$
f \mapsto\left(\int_{X}\|f(x)\|_{\mathscr{K}}^{2} d \mu(x)\right)^{1 / 2} .
$$

Denote by $\mathscr{L}(\mathscr{K})$ the space of linear bounded operators from $\mathscr{K}$ to $\mathscr{K}$. A function $A: X \rightarrow \mathscr{L}(\mathscr{K})$ is said to be measurable if $x \mapsto\langle A(x) \phi, \psi\rangle$ is measurable for all $\phi, \psi \in$ $\mathscr{K}$.

Let $L^{\infty}(X, d \mu ; \mathscr{L}(\mathscr{K}))$ be the space of bounded and measurable functions from $X$ to $\mathscr{L}(\mathscr{K})$. This is a Banach space with respect to the norm

$$
\|A\|_{\infty} \equiv \operatorname{ess} \sup \|A(x)\|_{\mathscr{L}(\mathscr{K})}
$$

Definition 2.20 ([RS78]). A bounded operator $A$ on $\mathscr{H}=\int_{X}^{\oplus} \mathscr{K} d \mu$ is said to be decomposable by the direct integral decomposition if and only if there is a function $A(\cdot)$ in $L^{\infty}(X, d \mu ; \mathscr{L}(\mathscr{K}))$ so that for all $\psi \in \mathscr{H}$,

$$
(A \psi)(x)=A(x) \psi(x)
$$

The $A(x)$ are called the fibers of $A$. One writes

$$
A=\int_{X}^{\oplus} A(x) d \mu(x) .
$$

From above definition, each decomposable operator is associated with some $A(\cdot)$ on $L^{\infty}(X, d \mu ; \mathscr{L}(\mathscr{K}))$. There is also inverse statement which will give an isometric isomorphism of $L^{\infty}(X, d \mu ; \mathscr{L}(\mathscr{K}))$ and decomposable operators.

Theorem 2.21 ( $\llbracket \mathrm{RS78}$, Theorem XIII.83]). If $A(\cdot)$ is in $L^{\infty}(X, d \mu ; \mathscr{L}(\mathscr{K}))$, then there is a unique decomposable operator $A \in \mathscr{L}(\mathscr{H})$ so that (2.5) holds. Moreover, $\|A\|_{\mathscr{L}(\mathscr{H})}=$ $\|A(\cdot)\|_{\infty}$

Remark 2.22 ([RS78]). The spaces of decomposable operators and $L^{\infty}(X, d \mu ; \mathscr{L}(\mathscr{K}))$ can be seen as algebras in the natural way. The space $\mathscr{A}$ is a subalgebra of $L^{\infty}(X, d \mu ; \mathscr{L}(\mathscr{K}))$ which consists of all decomposable operators whose fibers are all multiplies of the identity.

Theorem 2.23 ([RS78, Theorem XIII.84]). Let $\mathscr{H}=\int_{X}^{\oplus} \mathscr{K} d \mu(x)$, where $(X, \mu)$ is a $\sigma$-finite measure space and $\mathscr{K}$ is separable. Then, $A \in \mathscr{L}(\mathscr{H})$ if and only if A commutes with each operator in $\mathscr{A}$. 
Definition $2.24([$ RS78] $)$. A function $A(\cdot)$ from the measure space $X$ into self-adjoint operators (not necessary bounded) on a Hilbert space $\mathscr{K}$ is called measurable if and only if the function $(A(\cdot)+i)^{-1}$ is measurable. Given such a function, we define an operator $A$ on $\mathscr{H}=\int_{X}^{\oplus} \mathscr{K} d \mu(x)$ with domain

$$
D(A):=\left\{\psi \in \mathscr{H}: \psi(x) \in D(A(x)) \text { a.e, } \int_{X}\|A(x) \psi(x)\|_{\mathscr{K}}^{2} d x<\infty\right\}
$$

by

$$
(A \psi)(x)=A(x) \psi(x)
$$

One writes $A=\int_{X}^{\oplus} A(x) d \mu(x)$.

We have the following properties:

Theorem $2.25\left(\llbracket\right.$ RS78, Theorem XIII.85]). Let $A=\int_{X}^{\oplus} A(x) d \mu(x)$, where $A(\cdot)$ is mesurable and $A(x)$ is self-adjoint for each $x$. Then:

1) The operator A is self-adjoint.

2) A self-adjoint opearator $A$ on $\mathscr{H}$ has the form $A=\int_{X}^{\oplus} A(x) d \mu(x)$ if and only if $(A+i)^{-1}$ is a bounded decomposable operator.

3) For any Borel function $F$ on $\mathbb{R}$

$$
F(A)=\int_{X}^{\oplus} F(A(x)) d \mu(x) .
$$

4) $\lambda \in \sigma(A)$ if and only if for any $\epsilon>0$,

$$
\mu(x: \sigma(A(x)) \cap(\lambda-\epsilon, \lambda+\epsilon) \neq \varnothing)>0 .
$$

5) $\lambda$ is an eigenvalue of $A$ if an only if

$$
\mu(x: \lambda \text { is an eigenvalue of } A(x))>0 .
$$

6) If each $A(m)$ has purely absolutely continuous spectrum, then so does $A$.

7) Suppose that $B=\int_{X}^{\oplus} B(x) d \mu(x)$ with each self-adjoint operator $B(x)$. If $B$ is A-bounded with A-bound a, then a.e. $B(x)$ is $A(x)$-bounded with $A(x)$-bound $a(x) \leq a$. If $a<1$, then

$$
A+B=\int_{X}^{\oplus}(A(x)+B(x)) d \mu(x)
$$

is self-adjoint on $D(A)$. 
Remark 2.26. Part 6) of Theorem 2.25] shows a sufficient condition for purely absolute continuity of the spectrum for the direct integral. For periodic Schrödinger operators, each $A(x)$ has purely discrete spectrum but $A$ has purely absolutely continuous spectrum.

Theorem 2.27 ([ $\mathrm{RS78}$, Theorem XIII.86]). Let $(X, d \mu)$ be $[0,1]$ with Lebesque measure. Let $\mathscr{K}$ be a fixed separable infinite dimensional space and let $A=\int_{[0,1]}^{\oplus} A(x) d \mu(x)$ with each $A(x)$ self-adjoint. Suppose $\mathscr{K}$-valued functions $\left\{\psi_{j}(\cdot)\right\}_{j=1}^{\infty}$ are real analytic on $(0,1)$, continuous on $[0,1]$, and complex-valued functions $E_{j}(\cdot)$ are analytic in a neighborhood of $[0,1]$, so that:

i) No $E_{j}(\cdot)$ is constant.

ii) $A(x) \psi_{n}(x)=E_{j}(x) \psi_{j}(x)$ for all $x \in[0,1], j=1,2, \ldots$.

iii) For each $x \in X$, the set $\left\{\psi_{j}(\cdot)\right\}_{j=1}^{\infty}$ is a complete orthogonal basic for $\mathscr{K}$.

Then, A has purely absolutely continuous spectrum.

Example 2.28 ([RS78, Kuc16]). Using the same notation as in Section 2.1, the following direct integrals hold

$$
L^{2}\left(\mathbb{R}^{d}\right)=\int_{B}^{\oplus} L^{2}(W), \quad H^{s}\left(\mathbb{R}^{d}\right)=\int_{B}^{\oplus} H_{k}^{s}(W)
$$

Using Floquet-Bloch-Gelfand transform and the direct integral of $H^{2}\left(\mathbb{R}^{d}\right)$, the operator $H=-\Delta+V(x)$ can be represented by the direct integral as follow:

Theorem 2.29 ([RS78, Theorem XIII.97]). Suppose that $V(x)$ is piecewise continuous and periodic with respect to a lattice $\Gamma$ which is generated by a basis $\left\{a_{1}, a_{2}, \cdots, a_{d}\right\}$. Let $W, B$ be the fundamental region and the Brillouin zone. Then, the operator $H=$ $-\Delta+V(x)$ has a direct integral

$$
\mathcal{U} H \mathcal{U}^{-1}=\int_{B}^{\oplus} H(k) \frac{d k}{(2 \pi)^{d}}
$$

where $H(k)$ is the operator $-\Delta+V(x)$ on $W$ with the quasi-periodic boundary conditions

$$
\psi\left(x+a_{j}\right)=e^{i k a_{j}} \psi(x), \quad \frac{\partial \psi}{\partial a_{j}}\left(x+a_{j}\right)=e^{i k a_{j}} \frac{\partial \psi}{\partial a_{j}}(x)
$$

for all $x$ such that $x, x+a_{j} \in W$, i.e., $x$ belongs to a suitable face of $W$. 
Example 2.30 ([RS78], one dimensional example). Let $V$ be a piecewise continuous and periodic function with period 1 . For $k \in[0,2 \pi]$, define

$$
H(k)=\left(-\frac{d^{2}}{d x^{2}}+V(x)\right)_{k},
$$

an operator on $L^{2}[0,1]$ with the quasi-periodic boundary conditions

$$
\psi(1)=e^{i k} \psi(0), \quad \psi^{\prime}(1)=e^{i k} \psi^{\prime}(0) .
$$

Then,

$$
\mathcal{U}\left(-\frac{d^{2}}{d x^{2}}+V(x)\right) \mathcal{U}^{-1}=\int_{B}^{\oplus} H(k) \frac{d k}{2 \pi} .
$$

The operator $H(k)$ is self-adjoint and has purely discrete spectrum satisfying all conditions in Theorem 2.27, Therefore, $H=-\frac{d^{2}}{d x^{2}}+V$ has purely absolutely continuous spectrum.

\subsubsection{Band gap structure of the spectrum}

The Floquet-Bloch-Gelfand transform and the direct integral reduce the study of the spectrum of the operator $H$ on $\mathbb{R}^{d}$ to the study the spectrum of a self-adjoint elliptic operator $H(k)$ on the fundamental region $W$ with the quasi-periodic boundary condition with quasi-momentum $k$. Using compact embedding $H^{2}(W) \hookrightarrow L^{2}(W)$, it implies that the operator $H(k)$ has compact resolvent. Thus, for each $k \in B, H(k)$ has discrete spectrum consisting of eigenvalues $\lambda_{j}(k), j \in \mathbb{N}$ (counting multiplicities)

$$
\lambda_{1}(k) \leq \lambda_{2}(k) \leq \ldots
$$

and $\lambda_{j}(k) \rightarrow \infty$ as $j \rightarrow \infty$. The functions $\lambda_{j}(k), j \in \mathbb{N}$ are called band functions. The next theorem follows from analytic perturbation theory (see Appendix A.2).

Theorem 2.31 ([Kuc93, Kuc16]). The band function $\lambda_{j}(k)$ is continuous and piecewise analytic in $B$.

A consequence of Theorem 2.31, $\sigma(H)$ is the countable union of compact intervals

$$
\sigma(H)=\bigcup_{j=1}^{\infty}\left[\min _{k \in B} \lambda_{j}(k), \max _{k \in B} \lambda_{j}(k)\right]
$$

There might have some spectral gaps if these intervals do not overlap and touch, i.e

$$
\max _{k \in B} \lambda_{j}(k)<\min _{k \in B} \lambda_{j+1}(k)
$$


for some $j \in \mathbb{N}$.

The next theorem shows the structure of the spectrum of periodic Schrödinger operator.

Theorem 2.32 ([Tho73]). Suppose $V(x) \in L_{\mathrm{loc}}^{2}\left(\mathbb{R}^{d}\right)$ is periodic with respect to some lattice $\Gamma$. Then, the spectrum of $-\Delta+V(x)$ on $L^{2}\left(\mathbb{R}^{d}\right)$ is purely absolutely continuous.

Remark 2.33 ([Kuc16]). There are many differences between the spectrum of periodic operators in one and higher dimensions.

i) In one dimension,

- bands do not overlap, but they can touch,

- gaps exist for non-constant potentials and generically there are infinitely many gaps (see [Sim76]).

ii) In higher dimensions,

- bands can (and mostly do) overlap,

- gaps might be non-existent (e.g., for small potentials),

- the number of gaps is finite (see [Par08, PS10]).

Remark 2.34 ([RS78]). In one dimension, if only finitely many gaps are present, then $V(x)$ is real analytic as a function on $\mathbb{R}$.

In one dimension, the spectral bands of $-\frac{d^{2}}{d x^{2}}$ touch to cover the whole half-axis $[0, \infty)$. But in higher dimensions, the spectral bands of $-\Delta$ overlap to cover $[0, \infty)$.

Theorem 2.35 ([Par08, PS10]). For $d \geq 2$, spectral bands of Laplacian $-\Delta$ overlap, and thus the spectrum has no gaps. Moreover, the length of the overlap tends to infinity when one goes up along the spectrum.

Using perturbation theory, a consequence of Theorem 2.35 is

Theorem 2.36 ([Kuc16]). Let $d \geq 2$. If the $L^{\infty}\left(\mathbb{R}^{d}\right)$-norm of periodic potential $V(x)$ is sufficient small, then Schrödinger operator $H=-\Delta+V$ has no spectral gap.

Theorem 2.37 ([KS87]). The bottom of spectrum of periodic Schrödinger operator $H=$ $-\Delta+V(x)$ on $\mathbb{R}^{d}$ is attained by the non-degenerated minimum (i.e. non-degenerated Hessian) at $k=0$ of the lowest eigenvalue $\lambda_{1}(k)$ only. 


\subsubsection{Bloch waves}

Definition 2.38 ([Kuc93]). The eigenfunctions $\psi_{j}(\cdot, k)$ for $j \in \mathbb{N}$ of $H(k)$ in $L^{2}(W)$ extended to all $\mathbb{R}^{d}$ using the boundary condition $\psi\left(x+a_{j}, k\right)=e^{i k a_{j}} \psi(x, k)$ are called Bloch waves or Bloch functions with quasi-momentum $k$.

Theorem 2.39 ([Kuc93, Section 4.3]). Existence of a non-trivial bounded solution of a periodic elliptic equation $\mathrm{Hu}=\lambda u$ implies existence of a Bloch function with real quasi-momentum, and thus $\lambda \in \sigma(H)$.

Theorem 2.40 ([Kuc93, Kuc16]). The Bloch waves $\psi_{j}(x, k)$ of operator H( $\left.k\right)$ corresponding to eigenvalue $\lambda_{j}(k)$ can be chosen such that

(i) They are measurable functions in $k$.

(ii) For fixed $k$, they form the orthonormal basis of $L^{2}(W)$.

Theorem 2.41 ([Koh59]). In one dimension, the band functions are analytic in $k$ and Bloch waves can be chosen as analytic functions in $k$.

Remark 2.42. In higher dimension, the structure of band functions and Bloch waves is much more complicated because of crossing bands. But if there is no crossing, then it follows from the standard theory of perturbation.

Lemma 2.43 (〔Kat95, Kuc16]). Let $j \in \mathbb{N}, k \in B$. If $\lambda_{j}\left(k^{*}\right)$ is a simple eigenvalue of $H\left(k^{*}\right)$, then $\lambda_{j}(k)$ is real analytic function of $k$ for $k$ near $k^{*}$.

This is local analyticity of band functions. If there is a bands crossing, then we do not have the analyticity in general.

Theorem 2.44 ([RS78, Theorem XIII.98], eigenfunction expansion). For $f \in \mathscr{S}\left(\mathbb{R}^{d}\right)$, let

$$
\widetilde{f}(j, k)=\int_{\mathbb{R}^{d}} f(x) \overline{\psi_{j}(x, k)} d x .
$$

Then:
i) $\int_{\mathbb{R}^{d}}|f(x)|^{2} d x=\frac{1}{(2 \pi)^{d}} \sum_{j=1}^{\infty} \int_{B}|\widetilde{f}(j, k)|^{2} d k$.
ii) $f(x)=\frac{1}{(2 \pi)^{d}} \sum_{j=1}^{\infty} \int_{B} \widetilde{f}(j, k) \psi_{j}(x, k) d k$. 
iii) Extend $\sim$ to $L^{2}\left(\mathbb{R}^{d}\right)$ by continuity. Then,

$$
\widetilde{H f}(j, k)=\lambda_{j}(k) \widetilde{f}(j, k)
$$

for all $f \in D(H)$.

iv) $\sim \operatorname{maps} L^{2}\left(\mathbb{R}^{d}\right)$ onto $\underset{j=1}{\infty} L^{2}(B)$.

\subsubsection{Dispersion relation}

Definition 2.45 ([Kuc93]). The real dispersion relation (or the real Bloch variety) $B_{H}$ of the periodic Schrödinger operator $H$ is the subset of $\mathbb{R}_{k}^{d} \times \mathbb{R}$ defined as follow:

$$
\begin{array}{r}
B_{H}=\left\{(k, \lambda) \in \mathbb{R}^{d+1}: H u=\lambda u\right. \text { admits a non-trivial } \\
\text { Bloch wave with quasi-momentum } k\} .
\end{array}
$$

Remark 2.46. The complex dispersion (or complex Bloch variety) is defined analogously by allowing $k, \lambda$ to be complex. Then, $B_{H, \mathbb{C}} \subset \mathbb{C}^{d+1}$.

Proposition 2.47 ([Kuc16]). i) The real dispersion relation is $\Gamma^{*}$-periodic with respect to $k$. Therefore, it is enough to consider only over the Brillouin zone.

ii) The real dispersion relation is symmetric (even) with respect to the mapping $k \mapsto$ $-k$.

iii) The graph of the multi-valued mapping

$$
k \in \mathbb{R}^{d}\left(\mathbb{C}^{d}\right) \mapsto \sigma(H(k))
$$

coincides with the (complex) Bloch variety $B_{H}\left(B_{H, \mathbb{C}}\right)$ of $H$.

Theorem 2.48 ([Kuc93, Theorem 4.4.2]). The complex dispersion $B_{H, \mathbb{C}}$ is the set of all zeros of an entire function of order $d$ on $\mathbb{C}^{d+1}$. Increasing the order to $d+1$, one can achieve $\Gamma^{*}$-periodicity of this functions with respect to $k$.

The notion of Fermi surface is one of the most important in solid state physics.

Definition 2.49 ([Kuc93, Kuc16]). The real Fermi surface $F_{H, \lambda}$ of periodic operator $H$ at a scalar value $\lambda$ is the $\lambda$-level set of the real dispersion relation, i.e.,

$$
F_{H, \lambda}=\left\{k \in \mathbb{R}^{d}:(k, \lambda) \in B_{H}\right\}
$$


Similarly, the complex Fermi surface is defined

$$
F_{H, \mathbb{C}, \lambda}=\left\{k \in \mathbb{C}^{d}:(k, \lambda) \in B_{H, \mathbb{C}}\right\} .
$$

Proposition 2.50 ([Kuc93, Kuc16]). i) The Fermi surface is $\Gamma^{*}$-periodic.

ii) $F_{H, \lambda}$ is the zero set of an entire function of the same exponential order as for $B_{H}$ (see Theorem 2.48).

Theorem 2.51 ([Kuc16, Theorem 5.33]). Let $V \in L^{\infty}\left(\mathbb{R}^{d}\right)$ be periodic. Then, for any energy level $\lambda \in \mathbb{R}$, the real Fermi surface of operator $H=-\Delta+V$ has measure zero in $\mathbb{R}^{d}$.

Theorem 2.52 ([Bor46, Borg theorem]). Consider the Hill's operator $H=-\frac{d^{2}}{d x^{2}}+V(x)$ with periodic potential $V(x)$. Then, the following are equivalent:

i) The potential is constant.

ii) There are no spectral gaps.

iii) There exists an entire function whose graph $\lambda=f(k)$ belongs to the dispersion relation.

Remark 2.53 ([Kuc16]). In higher dimensions, the equivalence of $i$ ) and $i i)$ fails. In fact, if the potential is sufficiently small and bounded, then the periodic Schrödinger operator has no gap at all. The equivalence $i$ ) and iii) is proved in two dimension by Knörrer and Trubowitz [KT90]. 



\section{The one-dimensional case}

In this chapter, we recall Floquet theory for periodic differential operators MW66, Eas73]. A consequence is that the spectrum of such operators has the band gap structure. Sturm-Liouville theory [Tit58, Tit62] gives the integral formula for the solution of Hill's equation. From this formula, we derive radiation conditions in the one-dimensional case.

\subsection{Floquet theory for Hill's operator}

We consider Hill's operator

$$
H=-\frac{d^{2}}{d x^{2}}+V(x) \quad \text { on } L^{2}(\mathbb{R})
$$

where $V(x)$ is smooth real-valued and periodic with period 1, i.e., $V(x)=V(x+1)$ for all $x \in \mathbb{R}$.

\subsubsection{The discriminant}

The homogeneous Hill's equation has the form

$$
-u^{\prime \prime}+(V(x)-\lambda) u=0
$$

where $\lambda$ is the spectral parameter.

Remark 3.1. In general, we can consider equation

$$
\left\{P(x) u^{\prime}(x)\right\}^{\prime}+Q(x) u(x)=0
$$

where $P(x)$ and $Q(x)$ are smooth real-valued and periodic with the same period 1, $P(x)$ is nowhere zero. After suitable transformations, equation (3.3) has the form of equation (3.2). 
Definition 3.2 ([MW66]). Suppose $u_{1}, u_{2}$ are two solutions of the homogeneous Hill's equation (3.2). The Wronskian of $u_{1}, u_{2}$ is defined by

$$
W\left(u_{1}, u_{2}\right)=\operatorname{det}\left(\begin{array}{cc}
u_{1} & u_{2} \\
u_{1}^{\prime} & u_{2}^{\prime}
\end{array}\right)=u_{1} u_{2}^{\prime}-u_{2} u_{1}^{\prime} .
$$

Take the derivative with respect to $x$ of the Wronskian, we get

$$
\frac{d}{d x} W\left(u_{1}, u_{2}\right)=u_{1} u_{2}^{\prime \prime}-u_{2} u_{1}^{\prime \prime}=0
$$

Here, we use the equation (3.2) for $u_{1}$ and $u_{2}$. Therefore, the Wronskian is independent of the variable $x$. It only depends on the spectral parameter $\lambda$.

Definition 3.3 ([MW66], fundamental system). A fundamental system $u_{1}(x, \lambda)$ and $u_{2}(x, \lambda)$ of equation (3.2) is the system of solutions of (3.2) satisfying conditions

$$
\begin{aligned}
& u_{1}(0, \lambda)=1, \quad u_{1}^{\prime}(0, \lambda)=0, \\
& u_{2}(0, \lambda)=0, \quad u_{2}^{\prime}(0, \lambda)=1 .
\end{aligned}
$$

Remark 3.4. From the standard theory of ordinary differential equations (see [Tit58, Tit62]), two solutions $u_{1}, u_{2}$ are analytic with respect to $\lambda$.

Definition 3.5 ([MW66]). i) The monodromy matrix is defined by

$$
M(\lambda)=\left(\begin{array}{ll}
u_{1}(1, \lambda) & u_{2}(1, \lambda) \\
u_{1}^{\prime}(1, \lambda) & u_{2}^{\prime}(1, \lambda)
\end{array}\right)
$$

ii) The discriminant $D(\lambda)$ of Hill's equation (3.2) is defined as the trace of matrix $M(\lambda)$.

Remark 3.6. The determinant $\operatorname{det} M(\lambda)$ is the Wronskian of the fundamental system $u_{1}, u_{2}$ at $x=1$. Therefore, $\operatorname{det} M(\lambda)=1$ because the Wronskian is constant in $x$.

Definition 3.7 ([MW66]). i) The characteristic equation is

$$
\mu^{2}-D(\lambda) \mu+1=0
$$

ii) The characteristic exponent $k \in[0,2 \pi)$ satisfies

$$
e^{i k}=\mu_{1}, \quad e^{-i k}=\mu_{2},
$$

where $\mu_{1}, \mu_{2}$ are roots of (3.4). 
Theorem 3.8 ([MW66, Floquet theorem]). $\quad$ 1. If the roots $\mu_{1}, \mu_{2}$ of (3.4) are different from each other, then Hill's equation (3.2) has two linear independent solutions

$$
e^{i k x} p_{1}(x), \quad e^{-i k x} p_{2}(x)
$$

where $p_{1}(x), p_{2}(x)$ are periodic with period 1 .

2. If $\mu_{1}=\mu_{2}$, then (3.4) has a non-trivial solution which is periodic with period 1 (when $\mu_{1}=\mu_{2}=1$ ) or 2 (when $\mu_{1}=\mu_{2}=-1$ ). Let $p(x)$ denote such a periodic solution and let $u(x)$ be another solution linear independent of $p(x)$. Then, there exists a constant $\theta$ such that

$$
u(x+1)=\mu_{1} u(x)+\theta p(x)
$$

Moreover, $\theta=0$ is equivalent to

$$
D(\lambda)= \pm 2, \quad u_{2}(1, \lambda)=0, \quad u_{1}^{\prime}(1, \lambda)=0
$$

Remark 3.9 ([Eas73, MW66]). i) If $\lambda \in \mathbb{R}$ and $|D(\lambda)|<2$, then equation (3.4) has two different real roots. Thus all solutions of Hill's equation (3.2) are bounded.

ii) $D(\lambda)=2$ corresponds to the existence of a non-trivial solution of the so-called periodic eigenvalue problem

$$
\left\{\begin{array}{l}
-u^{\prime \prime}+V(x) u=\lambda u, \quad 0<x<1 \\
u(0)=u(1), \quad u^{\prime}(0)=u^{\prime}(1)
\end{array}\right.
$$

iii) $D(\lambda)=-2$ corresponds to the existence of a non-trivial solution of the so-called anti-periodic eigenvalue problem

$$
\left\{\begin{array}{l}
-u^{\prime \prime}+V(x) u=\lambda u, \quad 0<x<1 \\
u(0)=-u(1), \quad u^{\prime}(0)=-u^{\prime}(1)
\end{array}\right.
$$

iv) If $D(\lambda)>2$, then all non-trivial solutions of (3.2) are unbounded in $\mathbb{R}$.

Theorem 3.10 ([Eas73, Theorem 1.3.3]). Let $k$ be a positive integer. Then, equation (3.2) has a non-trivial solution with period $k$ if and only if there is an integer $l$ such that

$$
D(\lambda)=2 \cos (2 l \pi / k)
$$


Theorem 3.11 ([Eas73, Theorem 1.3.4]). If $V(x)$ is even, i.e., $V(x)=V(-x)$, then (3.2) has a non-trivial solution which is

- even and periodic with period 1 if and only if $u_{1}^{\prime}(1 / 2)=0$,

- odd and periodic with period 1 if and only if $u_{2}(1 / 2)=0$,

- even and periodic with period 2 if and only if $u_{1}(1 / 2)=0$,

- odd and periodic with period 2 if and only if $u_{2}^{\prime}(1 / 2)=0$.

\subsubsection{Band gap structure of the spectrum}

In this section, we recall the definition of stable solutions and stable intervals from [MW66]. Using Floquet theory, the discriminant is analyzed and the band gap structure of the spectrum is derived.

Definition 3.12 ([MW66]). If all solutions of Hill's equation (3.2) are bounded, then we say that they are stable. Otherwise, we say that they are unstable.

Remark (3.9) leads to criteria for the stability of the solutions of Hill's equation.

i) If $|D(\lambda)|>2$, then all solutions is unstable.

ii) If $|D(\lambda)|<2, \lambda \in \mathbb{R}$, then all solutions are stable.

iii) If $|D(\lambda)|=2$, then in general the solutions are stable except the case the equation $D(\lambda)= \pm 2$ has double solutions.

Floquet theory reduces the study of the spectrum of Hill's operator (3.1) to the spectral problem for $k \in B=[-\pi, \pi]$

$$
\left\{\begin{array}{lr}
-u^{\prime \prime}+V(x) u=\lambda u, & 0<x<1, \\
u(0)=e^{i k} u(1), & u^{\prime}(0)=e^{i k} u^{\prime}(1) .
\end{array}\right.
$$

Theorem 3.13 ([Eas73]). $\lambda$ is an eigenvalue of (3.5) if and only if

$$
D(\lambda)=2 \cos k
$$

As a consequence, one obtains 
Theorem 3.14 ([Kuc16, Theorem 1.14]). The real (complex) dispersion relation is described as the set of real (complex) solutions $(k, \lambda)$ of the equation

$$
D(\lambda)=2 \cos k
$$

Theorem 3.15 ([MW66, Theorem 2.2]). The discriminant $D(\lambda)$ is an entire function of complex variable $\lambda$. Its orders of growth for $|\lambda| \rightarrow \infty$ is exactly $1 / 2$. More precisely, there exists a positive constant $M$ such that

$$
|D(\lambda)| \exp (-M \sqrt{|\lambda|})
$$

is bounded for all $\lambda$, and there exists a positive constant $m$ such that $\lambda$ real and $\lambda \rightarrow-\infty$ implies

$$
|D(\lambda)| \exp (-m \sqrt{|\lambda|}) \rightarrow \infty
$$

As a consequence of Theorem 3.15, one has

Corollary 3.16 ([MW66]). $D(\lambda)=2$ has infinitely many real roots

$$
\lambda_{0}<\lambda_{1} \leq \lambda_{2}<\lambda_{3} \leq \lambda_{4}<\cdots
$$

and the equation $D(\lambda)=-2$ has infinitely many real roots

$$
\mu_{0} \leq \mu_{1}<\mu_{2} \leq \mu_{3}<\cdots
$$

They satisfy inequalities

$$
\lambda_{0}<\mu_{0} \leq \mu_{1}<\lambda_{1} \leq \lambda_{2}<\mu_{2} \leq \mu_{3}<\lambda_{3} \leq \lambda_{4}<\cdots,
$$

and $\lambda_{n} \rightarrow \infty, \mu_{n} \rightarrow \infty$ as $n \rightarrow \infty$.

Theorem 3.17 ([Eas73, MW66]). i) The spectrum of the operator $H$ on $L^{2}(\mathbb{R})$ is the union of closed intervals

$$
\left[\lambda_{0}, \mu_{0}\right], \quad\left[\mu_{1}, \lambda_{1}\right], \quad\left[\lambda_{2}, \mu_{2}\right], \quad\left[\mu_{3}, \lambda_{3}\right], \cdots .
$$

ii) There might have gaps between the spectrum of the operator $H$

$$
\left(\mu_{0}, \mu_{1}\right), \quad\left(\lambda_{1}, \lambda_{2}\right), \quad\left(\mu_{2}, \mu_{3}\right), \quad\left(\lambda_{3}, \lambda_{4}\right), \cdots
$$

Gaps disappear if equation $D(\lambda)= \pm 2$ has double solutions at some points. 
Remark 3.18 ([Eas73, MW66]). i) $D(\lambda)$ decreases monotonically on $\left(-\infty, \mu_{0}\right)$ and $\left[\lambda_{j}, \mu_{j}\right]$, and increases monotonically on $\left[\mu_{j}, \lambda_{j}\right], j \in \mathbb{N}$.

ii) If $\mu_{2 j} \neq \mu_{2 j+1}$, then $D^{\prime}\left(\mu_{2 j}\right) \neq 0, D^{\prime}\left(\mu_{2 j+1}\right) \neq 0, j \in \mathbb{N}$. The same holds for $\lambda_{2 j-1} \neq \lambda_{2 j}, j \in \mathbb{N}^{*}$. If $\mu_{2 j}=\mu_{2 j+1}, j \in \mathbb{N}$ or $\lambda_{2 j-1}=\lambda_{2 j}, j \in \mathbb{N}^{*}$, then $D^{\prime}(\lambda)=0$ at these points, but $D^{\prime \prime}(\lambda)$ is not zero.

Theorem 3.19 ([Eas73, RS78]). The pure point and singular continuous spectrum of Hill's operator are empty. Therefore, the spectrum is purely absolutely continuous.

Definition 3.20 ([MW66], coexistence). If two linearly independent solutions of Hill's equation (3.2) are of period $\pi$ or $2 \pi$, it is said to be an instance of coexistence.

Remark 3.21 ([MW66]). The coexistence of periodic solutions of period $\pi$ or $2 \pi$ is equivalent respectively to the existence of a double root of equation $D(\lambda)=2$ or $D(\lambda)=$ -2 .

Example 3.22 ([MW66]). Consider Mathieu's equation

$$
-u^{\prime \prime}(x)-(\lambda-2 \alpha \cos 2 x) u(x)=0, \quad x \in \mathbb{R}
$$

with a non-zero real constant $\alpha$. Then, there is no point $\lambda \in \mathbb{R}$ of coexistence.

Example 3.23 ([MW66]). Consider Whittaker-Hill's equation

$$
-u^{\prime \prime}(x)-\left(\lambda+4 m q \cos 2 x+2 q^{2} \cos 4 x\right) u(x)=0
$$

with $m$ is an integer and $q$ is real. Then, there is coexistence.

\subsection{Radiation conditions for Hill's equation}

Consider Hill's equation

$$
-u^{\prime \prime}+(V(x)-\lambda) u=f(x)
$$

where $\lambda$ is in the spectrum of the operator $H$, not an edge of any band, i.e., $\lambda \neq \lambda_{j}, \lambda \neq$ $\mu_{j}, j \in \mathbb{N}$, and $f \in C_{\mathrm{c}}^{\infty}(\mathbb{R})$.

Because the domain is unbounded, we would like to find additional conditions at infinity to make (3.6) well-posed. This can be done by using the limiting absorption 
principle. Namely, one adds a small absorption $i \epsilon, \epsilon>0$ to the spectral parameter $\lambda$, then (3.6) becomes

$$
-u^{\prime \prime}+(V(x)-\lambda-i \epsilon) u=f(x) .
$$

The corresponding homogeneous Hill's equation is

$$
-u^{\prime \prime}+(V(x)-\lambda-i \epsilon) u=0 .
$$

From the spectral theory, equation (3.7) has a unique $L^{2}$-solution. We want to find a representation of this solution. We will use Floquet theorem 3.8 and Sturm-Liouville theory [Tit62, Tit58] to get two independent solutions of the homogeneous Hill's equation (3.8).

Let $u_{1}(x, \lambda+i \epsilon), u_{2}(x, \lambda+i \epsilon)$ be a fundamental system of (3.8). Then, $u_{1}, u_{2}$ are entire functions of $\lambda$. The discriminant $D(\lambda+i \epsilon)$ is also an entire function of order exactly $1 / 2$. From Floquet theorem 3.8, there exist the $k \in \mathbb{C}$ depending on $\lambda$ and $\epsilon$ such that (3.8) has two linear independent solutions $e^{i k x} p_{1}(x)$ and $e^{-i k x} p_{2}(x)$, where $p_{1}, p_{2}$ are periodic with period 1. If $\epsilon>0$, then the discriminant $D(\lambda+i \epsilon)$ is non-real. Therefore, $k$ is also non-real. We could choose $k$ such that $\operatorname{Im} k>0$. Sturm-Liouville theory derives two linear independent solutions of (3.8).

Lemma 3.24. Let $\lambda$ be in the spectrum of operator $H$, not an edge of any band. Then, $u_{2}(1, \lambda) \neq 0$. Consequently, for $\epsilon$ small enough, $u_{2}(1, \lambda+i \epsilon) \neq 0$.

Proof. Assuming that $u_{2}(1, \lambda)=0$. The Wronskian of the fundamental system $u_{1}(x, \lambda)$, $u_{2}(x, \lambda)$ is equal to 1 . Therefore,

$$
u_{1}(1, \lambda) u_{2}^{\prime}(1, \lambda)-u_{1}^{\prime}(1, \lambda) u_{2}(1, \lambda)=1 .
$$

Using $u_{2}(1, \lambda)=0$, it implies

$$
u_{1}(1, \lambda) u_{2}^{\prime}(1, \lambda)=1 \text {. }
$$

Because $\lambda \in \sigma(H)$ is not an edge of any band, it implies

$$
|D(\lambda)|=\left|u_{1}(1, \lambda)+u_{2}^{\prime}(1, \lambda)\right|<2
$$

From (3.9) and (3.10), we get a contradiction. Therefore, $u_{2}(1, \lambda) \neq 0$. The last statement follows from the analyticity of $u_{2}$ with respect to $\lambda$. 
The next theorem gives explicit formulas of two independent solutions of homogeneous equation which are used to derive a integral representation of a solution of inhomogeneous equation.

Theorem 3.25 ([Tit58, Chapter XXI]). There exist two independent solutions of (3.8)

$$
\begin{aligned}
& \psi_{1, \epsilon}(x, \lambda)=u_{1}(x, \lambda+i \epsilon)+m_{1, \epsilon} u_{2}(x, \lambda+i \epsilon)=e^{i k x} p_{1}(x) \in L^{2}(0, \infty), \\
& \psi_{2, \epsilon}(x, \lambda)=u_{1}(x, \lambda+i \epsilon)+m_{2, \epsilon} u_{2}(x, \lambda+i \epsilon)=e^{-i k x} p_{2}(x) \in L^{2}(-\infty, 0),
\end{aligned}
$$

where

$$
\begin{aligned}
& m_{1, \epsilon}=\frac{u_{2}^{\prime}(1, \lambda+i \epsilon)-u_{1}(1, \lambda+i \epsilon)}{2 u_{2}(1, \lambda+i \epsilon)}-\frac{\sqrt{D^{2}(\lambda+i \epsilon)-4}}{2 u_{2}(1, \lambda+i \epsilon)} \\
& m_{2, \epsilon}=\frac{u_{2}^{\prime}(1, \lambda+i \epsilon)-u_{1}(1, \lambda+i \epsilon)}{2 u_{2}(1, \lambda+i \epsilon)}+\frac{\sqrt{D^{2}(\lambda+i \epsilon)-4}}{2 u_{2}(1, \lambda+i \epsilon)} .
\end{aligned}
$$

Here the branch of the square root is chosen from the condition $\sqrt{D^{2}(\lambda)-4}>0$ for $\lambda<\lambda_{0}$.

Remark 3.26. $m_{1, \epsilon}, m_{2, \epsilon}$ have branch points of square root type at $\lambda_{j}, \mu_{j}$ for all $j \in \mathbb{N}$. They are analytic in the upper (lower) half plane. Therefore, we could take limit as $\epsilon \rightarrow+0(\epsilon \rightarrow-0)$.

The next theorem gives an integral representation for unique $L^{2}$-solution of (3.7).

Theorem 3.27. The unique $L^{2}$-solution of (3.7) has the formula

$$
u_{\epsilon}(x, \lambda)=\frac{\psi_{1, \epsilon}(x, \lambda)}{\omega_{\epsilon}(\lambda)} \int_{-\infty}^{x} \psi_{2, \epsilon}(\xi, \lambda) f(\xi) d \xi+\frac{\psi_{2, \epsilon}(x, \lambda)}{\omega_{\epsilon}(\lambda)} \int_{x}^{\infty} \psi_{1, \epsilon}(\xi, \lambda) f(\xi) d \xi
$$

where $\omega_{\epsilon}(\lambda)=m_{2, \epsilon}-m_{1, \epsilon}$ is equal to the Wronskian of $\psi_{1, \epsilon}, \psi_{2, \epsilon}$.

Proof. The proof is based on [Tit62, Chapter II] and [Tit58, Chapter XXI] except $L^{2}$ solution part.

First, we show that $\omega_{\epsilon}(\lambda)=m_{2, \epsilon}-m_{1, \epsilon}=W\left(\psi_{1, \epsilon}, \psi_{2, \epsilon}\right)$. By direct computation,

$$
\begin{aligned}
W\left(\psi_{1, \epsilon}, \psi_{2, \epsilon}\right) & =\psi_{1, \epsilon} \psi_{2, \epsilon}^{\prime}-\psi_{1, \epsilon}^{\prime} \psi_{2, \epsilon} \\
& =\left(u_{1}+m_{1, \epsilon} u_{2}\right)\left(u_{1}^{\prime}+m_{2, \epsilon} u_{2}^{\prime}\right)-\left(u_{1}^{\prime}+m_{1, \epsilon} u_{2}^{\prime}\right)\left(u_{1}+m_{2, \epsilon} u_{2}\right) \\
& =\left(m_{2, \epsilon}-m_{1, \epsilon}\right)\left(u_{1} u_{2}^{\prime}-u_{1}^{\prime} u_{2}\right)=m_{2, \epsilon}-m_{1, \epsilon} .
\end{aligned}
$$

In the last step, we use $W\left(u_{1}, u_{2}\right)=1$. 
Differentiate (3.11) twice, we get

$$
u_{\epsilon}^{\prime}=\frac{\psi_{1, \epsilon}^{\prime}(x, \lambda)}{\omega_{\epsilon}(\lambda)} \int_{-\infty}^{x} \psi_{2, \epsilon}(\xi, \lambda) f(\xi) d \xi+\frac{\psi_{2, \epsilon}^{\prime}(x, \lambda)}{\omega_{\epsilon}(\lambda)} \int_{x}^{\infty} \psi_{1, \epsilon}(\xi, \lambda) f(\xi) d \xi
$$

and

$$
\begin{array}{r}
u_{\epsilon}^{\prime \prime}=\frac{\psi_{1, \epsilon}^{\prime \prime}(x, \lambda)}{\omega_{\epsilon}(\lambda)} \int_{-\infty}^{x} \psi_{2, \epsilon}(\xi, \lambda) f(\xi) d \xi+\frac{\psi_{2, \epsilon}^{\prime \prime}(x, \lambda)}{\omega_{\epsilon}(\lambda)} \int_{x}^{\infty} \psi_{1, \epsilon}(\xi, \lambda) f(\xi) d \xi+ \\
+\frac{\psi_{1, \epsilon}^{\prime} \psi_{2, \epsilon}-\psi_{1, \epsilon} \psi_{2, \epsilon}^{\prime}}{\omega_{\epsilon}(\lambda)} f(x)=(V(x)-\lambda-i \epsilon) u_{\epsilon}-f(x) .
\end{array}
$$

Therefore, $u_{\epsilon}$ satisfies (3.7).

Next, we prove that $u_{\epsilon} \in L^{2}(\mathbb{R})$. Suppose supp $f \subseteq[m, M]$. Consider the first part of (3.11), the second part is proved similarly. Let $\chi$ be the characteristic function. Then,

$$
g(x)=\frac{\psi_{1, \epsilon}(x, \lambda)}{\omega_{\epsilon}(\lambda)} \int_{-\infty}^{x} \psi_{2, \epsilon}(\xi, \lambda) f(\xi) d \xi=\left(\chi_{[m, M]}(x)+\chi_{(-\infty, m)}(x)+\chi_{(M, \infty)}(x)\right) g(x) .
$$

Because $g(x)$ is smooth, it implies $\chi_{[m, M]} g \in L^{2}(\mathbb{R})$. Moreover, $f(x)=0$ for $x<m$, therefore $\chi_{(-\infty, m)} g=0$. The last term $\chi_{(M, \infty)} g$ is also in $L^{2}(\mathbb{R})$ because $\chi_{(M, \infty)} \psi_{1, \epsilon} \in L^{2}(\mathbb{R})$ and

$$
\int_{-\infty}^{\infty} \psi_{2, \epsilon}(\xi, \lambda) f(\xi) d \xi \quad \text { is finite. }
$$

It completes the proof of Theorem 3.27 .

Remark 3.28. The function $\sqrt{D^{2}(\lambda)-4}$ can be extended analytically to the two-sheet Riemann surface $\Lambda$. This Riemann surface is obtained by gluing together two copies of the $\mathbb{C} \backslash \sigma$ along the spectrum. Here, the upper side of the band $g_{n}$ of the first sheet is glued to the lower side of the band $g_{n}$ and conversely.

The function $k$ depending on $\lambda$ is called the quasi-momentum. It is determined by $k(\lambda)=\arcsin \left(i \sqrt{D^{2}(\lambda)-4} / 2\right)$ (see [Fir75] for more details).

Recall Hill's equation

$$
-u^{\prime \prime}+(V(x)-\lambda) u=f(x)
$$

where $f \in C_{\mathrm{c}}^{\infty}(\mathbb{R})$. The interesting problem is when $\lambda$ is in the interior of the spectrum of the operator $H$. Here, we want to find radiation conditions for (3.12). The method is to use the limiting absorption principle.

Theorem 3.29 (Radiation condition for outgoing solution). Let $\lambda$ be in the spectrum of the operator $H$, not an edge of any band. Suppose supp $f \subseteq[m, M]$, then the limit $u(x, \lambda):=\lim _{\epsilon \rightarrow+0} u_{\epsilon}(x, \lambda)$ in $L_{\mathrm{loc}}^{2}(\mathbb{R})$ exists, and $u(x, \lambda)$ satisfies 
- If $x>M$, then

$$
u(x, \lambda)=C_{1} e^{i k x} p_{1}(x)
$$

- If $x<m$, then

$$
u(x, \lambda)=C_{2} e^{-i k x} p_{2}(x)
$$

Here $k(\lambda+i \epsilon) \rightarrow k$ as $\epsilon \rightarrow+0, p_{1}(x), p_{2}(x)$ are periodic with period 1, and $C_{1}, C_{2}$ are constant depending on $\lambda$. Due to periodicity, we can normalize such that $k \in[0,2 \pi)$.

Proof. From the formula (3.11), we have

$$
u_{\epsilon}(x, \lambda)=\frac{\psi_{1, \epsilon}(x, \lambda)}{\omega_{\epsilon}(\lambda)} \int_{-\infty}^{x} \psi_{2, \epsilon}(\xi, \lambda) f(\xi) d \xi+\frac{\psi_{2, \epsilon}(x, \lambda)}{\omega_{\epsilon}(\lambda)} \int_{x}^{\infty} \psi_{1, \epsilon}(\xi, \lambda) f(\xi) d \xi
$$

where $\psi_{1, \epsilon}(x, \lambda)=e^{i k(\lambda+i \epsilon) x} p_{1, \epsilon}(x), \psi_{2, \epsilon}(x, \lambda)=e^{-i k(\lambda+i \epsilon) x} p_{2, \epsilon}(x)$ for periodic functions $p_{1, \epsilon}(x), p_{2, \epsilon}(x)$ with period 1 .

Because $\psi_{1, \epsilon}(x, \lambda), \psi_{2, \epsilon}(x, \lambda)$ are analytic with respect to $\lambda$, it implies that $\psi_{1, \epsilon}(x, \lambda) \rightarrow$ $\psi_{1}(x, \lambda), \psi_{2, \epsilon}(x, \lambda) \rightarrow \psi_{2}(x, \lambda)$ as $\epsilon \rightarrow+0$ and $\left\{\psi_{1}, \psi_{2}\right\}$ is the fundamental system of

$$
-u^{\prime \prime}+(V(x)-\lambda) u=0
$$

Moreover, $\omega_{\epsilon}(\lambda) \rightarrow \omega(\lambda)$ as $\epsilon \rightarrow+0$, where $\omega(\lambda)=W\left(\psi_{1}, \psi_{2}\right) \neq 0$. The quasimomentum $k(\lambda+i \epsilon)$ tends to $k$ as $\epsilon \rightarrow+0$.

The limit $u(x, \lambda)=\lim _{\epsilon \rightarrow+0} u_{\epsilon}(x, \lambda)$ exists and has the formula

$$
u(x, \lambda)=\frac{\psi_{1}(x, \lambda)}{\omega(\lambda)} \int_{-\infty}^{x} \psi_{2, \epsilon}(\xi, \lambda) f(\xi) d \xi+\frac{\psi_{2}(x, \lambda)}{\omega(\lambda)} \int_{x}^{\infty} \psi_{1}(\xi, \lambda) f(\xi) d \xi
$$

Furthermore, $u(x, \lambda)$ is a smooth solution of (3.12).

- If $x>M$, then

$$
u(x, \lambda)=\frac{\psi_{1}(x, \lambda)}{\omega(\lambda)} \int_{-\infty}^{x} \psi_{2}(\xi, \lambda) f(\xi) d \xi
$$

Because $\lambda$ is not an edge of any band, therefore the Wronskian $\omega(\lambda) \neq 0$. The integral in (3.16) does not depend on $x$ because $f(x)=0$ for $x>M$. It implies

$$
u(x, \lambda)=C_{1} e^{i k x} p_{1}(x), \quad x>M,
$$

where $k=k(\lambda+i 0)$. 
- Similarly, when $x<m$, the solution has the form

$$
u(x, \lambda)=\frac{\psi_{2}(x, \lambda)}{\omega(\lambda)} \int_{x}^{\infty} \psi_{1}(\xi, \lambda) f(\xi) d \xi
$$

and satisfies

$$
u(x, \lambda)=C_{2} e^{-i k x} p_{2}(x), \quad x<m .
$$

Remark 3.30. The conditions (3.13), (3.14) are called radiation conditions for the outgoing solution. Next, we prove the unique solvibility of Hill's equation under these radiation conditions.

Theorem 3.31 (Uniqueness for outgoing solution). Suppose $\lambda$ is in the interior of the spectrum $\sigma(H)$ and not an edge of any band. Then, equation (3.12) with the radiation conditions (3.13) and (3.14) has a unique solution in $L_{\mathrm{loc}}^{2}(\mathbb{R})$.

Proof. Suppose equation (3.12) has two solutions $u_{1}(x), u_{2}(x)$ satisfying the radiation conditions (3.13) and (3.14). Then, $u(x)=u_{1}(x)-u_{2}(x)$ is a solution of the homogeneous equation $-u^{\prime \prime}+(V(x)-\lambda) u=0$ and it also satisfies the radiation conditions. We will prove that $u(x) \equiv 0$.

Indeed, suppose $\left\{e^{i k x} q_{1}(x), e^{-i k x} q_{2}(x)\right\}$, where $q_{1}, q_{2}$ are periodic with period 1 is the fundamental system of (3.15). Then, solution $u(x)$ of the homogeneous equation 3.15 has the form

$$
u(x)=B_{1} e^{i k x} q_{1}(x)+B_{2} e^{-i k x} q_{2}(x),
$$

where $B_{1}, B_{2}$ only depend on $k$.

For $x>M$, from condition (3.13) we have

$$
u(x)=C_{1} e^{i k x} p_{1}(x)
$$

where $p_{1}(x)$ is periodic with period 1 .

From (3.17) (3.18), it implies for $x>M$,

$$
B_{1} e^{i k x} q_{1}(x)+B_{2} e^{-i k x} q_{2}(x)=C_{1} e^{i k x} p_{1}(x) .
$$

Multiply both sides of this equation with $e^{i k x}$ and subtract, we get

$$
e^{2 i k x}\left\{B_{1} q_{1}(x)-C_{1} p_{1}(x)\right\}+B_{2} q_{2}(x)=0 .
$$


Replace $x$ by $x+1$ and using the periodicity, it implies

$$
\left(e^{2 i k}-1\right) B_{2} p_{1}(x)=0
$$

Because $\lambda$ is not the end point of band, that means $k \neq 0, k \neq \pi$, it implies $e^{2 i k} \neq 1$, and therefore $B_{2}=0$.

Similarly, for the case $x<m$, we obtain $B_{1}=0$. That means $u(x) \equiv 0$.

If we take the limit $u_{\epsilon}(x)$ as $\epsilon \rightarrow-0$ instead of $\epsilon \rightarrow+0$, then we get the incoming solution. In this case, $k(\lambda+i \epsilon) \rightarrow-k$ as $\epsilon \rightarrow-0$. We obtain radiation conditions for the incoming solution.

Theorem 3.32 (Radiation condition for incoming solution). Let $\lambda$ be in the spectrum of the operator $H$, not an edge of any band. Suppose supp $f \subseteq[m, M]$, then the limit $u(x, \lambda):=\lim _{\epsilon \rightarrow-0} u_{\epsilon}(x, \lambda)$ in $L_{\text {loc }}^{2}(\mathbb{R})$ exists, and $u(x, \lambda)$ satisfies

- If $x>M$, then

$$
u(x, \lambda)=C_{1} e^{-i k x} p_{1}(x)
$$

- If $x<m$, then

$$
u(x, \lambda)=C_{2} e^{i k x} p_{2}(x)
$$

Here, $k(\lambda+i \epsilon) \rightarrow-k$ as $\epsilon \rightarrow-0, p_{1}(x), p_{2}(x)$ are periodic with period $1 ; C_{1}, C_{2}$ are constant depending on $\lambda$.

Proof. It is similar to Theorem 3.29. Note that $\lim _{\varepsilon \rightarrow-0} k(\lambda+i \epsilon)=-k$.

Remark 3.33. These two conditions (3.19) and (3.20) are called radiation conditions for the incoming solution. We also get unique solvability for Hill's equation satisfying radiation conditions (3.19) and (3.20).

Theorem 3.34 (Uniqueness for incoming solution). Suppose $\lambda$ is in the spectrum and not an edge of any band. Then, equation (3.12) with radiation conditions (3.19) and (3.20) has a unique solution in $L_{\mathrm{loc}}^{2}(\mathbb{R})$

Proof. Similar to Theorem 3.31 . 


\section{The higher-dimensional case}

In this chapter, we will adapt the results to the higher-dimensional case. The limiting absorption principle in the distribution sense is recalled from [ $\operatorname{Rad} 15]$ and then adapted to the case of the Schrödinger operator with a smooth periodic potential. Then, using integral representation of solution (see Chapter2), we will deal with oscillatory integrals in higher dimensions. These integrals can be estimated by using analytic perturbation theory (see Appendix A.2 and the stationary phase method (see Appendix A.3). The main method comes from articles [MT06, KR12, KKR17]. We use the idea in [KR12, KKR17] to decompose the resolvent of periodic Schrödinger operator, then use some estimates from [MT06] to derive the asymptotic expansion of solutions to the periodic Schrödinger equation.

\subsection{The limiting absorption principal for periodic oper- ators}

In this section, we recall the limiting absorption principal from [ $\operatorname{Rad} 15]$. Consider a periodic operator $H$ with respect to some lattice $\Gamma$ on $L^{2}\left(\mathbb{R}^{d}\right)$. The operator $H$ can be written as the direct integral

$$
H=\int_{B}^{\oplus} H(k) d k,
$$

where $B$ is the Brillouin zone, $H(k)$ is formally the same as $H$, but acts on $L^{2}(W)$ ( $W$ is the fundamental region of $\Gamma$ ) with $k$-quasi-periodic condition (see Chapter2). Moreover, $H(k)$ has compact resolvent. Thus, there are sequences of the band functions $\lambda_{j}(k)$ and corresponding Bloch waves $\psi_{j}(x, k), j \in \mathbb{N}$ satisfying

$$
\begin{aligned}
& H(k) \psi_{j}(\cdot, k)=\lambda_{j}(k) \psi_{j}(\cdot, k), \quad j \in \mathbb{N}, k \in B, \\
& \psi_{j}(x+n, k)=e^{i k \cdot n} \psi_{j}(x, k), \quad n \in \Gamma, x \in W .
\end{aligned}
$$


In [Rad15], the author made the following general conditions on the band functions and Bloch waves

i) $\lambda_{j}(k), j \in \mathbb{N}$ are $B$-periodic (i.e., having the same values on opposite faces of $B$ ), real-valued Lipschitz functions.

ii) $k \mapsto \psi_{j}(k) \in L^{2}(W)$ is measurable, and for each $k \in B,\left\{\psi_{j}(\cdot, k), j \in \mathbb{N}\right\}$ is an orthonormal basis of $L^{2}(W)$.

Remark 4.1. These two above conditions are fulfilled for many operators in mathematical physics. Periodic Schrödinger operators satisfy all conditions (see Chapter2).

The decomposition of $H$ into the direct integral and the complete orthonormal $\psi_{j}(\cdot, k), j \in$ $\mathbb{N}$ for $L^{2}(W)$ imply the following integral representation:

Proposition $4.2([\underline{\operatorname{Rad} 15]})$. For $u \in D(H)$,

$$
H u(x)=\frac{1}{(2 \pi)^{d}} \sum_{j=1}^{\infty} \int_{B} \lambda_{j}(k)\left\langle U u(., k), \psi_{j}(., k)\right\rangle_{L^{2}(W)} \psi_{j}(x, k) d k,
$$

where the series converges in $L^{2}\left(\mathbb{R}^{d}\right)$.

Next, consider equation

$$
H u-\lambda u=f
$$

where $f(x) \in L^{2}\left(\mathbb{R}^{d}\right)$.

Definition 4.3 ([ $\operatorname{Rad10}$, Distribution solution]). A distribution $u$ acting on a test function $\phi(\lambda, x) \in C_{\mathrm{c}}^{\infty}\left(\mathbb{R} \times \mathbb{R}^{d}\right)$ is called a distribution solution of (4.1) if it is locally integrable on $\mathbb{R} \times \mathbb{R}^{d}$ and for all $\phi \in C_{\mathrm{c}}^{\infty}\left(\mathbb{R} \times \mathbb{R}^{d}\right)$,

$$
u[(H-\lambda) \phi]=\int_{\mathbb{R}} \int_{\mathbb{R}^{d}} f(x) \phi(\lambda, x) d x d \lambda .
$$

Using the integral representation of the solution it can be shown that the limiting absorption principle is valid for regular values which are defined as follow:

Definition 4.4 $([\operatorname{Rad} 15]) . \quad$ i) $\lambda \in \mathbb{R}$ is called a regular value of $\lambda_{s}$ if for every $k \in B$ such that $\lambda_{s}(k)=\lambda$, then $\nabla \lambda_{s}(k) \neq 0$.

ii) The set of all regular values is defined by

$$
\mathcal{R}=\left\{\lambda \in \mathbb{R}: \lambda \text { is a regular value of } \lambda_{s} \text { for all } s\right\} .
$$


Let $I_{0} \subset \mathcal{R}$ be compact and $I \subset I_{0}$ an open interval. Let

$$
\begin{aligned}
& V=\left\{k \in B: \lambda_{j}(k) \in I_{0} \text { for some } j \in \mathbb{N}\right\}, \\
& r=\max \left\{j \in \mathbb{N}: \lambda_{j}(B) \cap I_{0} \neq \varnothing\right\} .
\end{aligned}
$$

Theorem 4.5 ( $\operatorname{Rad15}$, Theorem 2.13]). The limiting absorption principle holds for the regular value $\lambda$, that means

$$
u(\lambda, x)=\lim _{\varepsilon \rightarrow+0}(H-\lambda-i \varepsilon)^{-1} f
$$

exists in $L^{2}\left(I ; L^{2}\left(\mathbb{R}^{d}, \omega(x) d x\right)\right)$ for some weighted function $\omega(x)$ and $u$ is the distribution solution of (4.1). Here,

$$
\begin{aligned}
u(x, \lambda)=\frac{1}{|B|} \sum_{j \in \mathbb{N}} \int_{B \backslash V} \frac{\left\langle\mathcal{U} f(., k), \psi_{j}(., k)\right\rangle_{L^{2}(W)}}{\lambda_{j}(k)-\lambda} \psi_{j}(x, k) d k- & \\
& -\pi \sum_{j \in \mathbb{N} ; j \leq r} \mathbb{H} h_{j}(\lambda, x)-i h_{j}(\lambda, x),
\end{aligned}
$$

where $\mathbb{H}$ is the Hilbert transform with respect to $\lambda$, and

$$
h_{j}(x, \lambda)=\frac{1}{(2 \pi i)^{d}} \int_{\Gamma_{j}(\lambda)} \frac{\left\langle\mathcal{U} f(., k), \psi_{j}(., k)\right\rangle_{L^{2}(W)}}{\left|\nabla \lambda_{j}(k)\right|} \psi_{j}(x, k) d k
$$

with $\Gamma_{j}(\lambda)=\left\{k \in B: \lambda_{j}(k)=\lambda\right\}$.

\subsection{Radiation conditions in higher dimensions}

In this section, we are interested in the Schrödinger equation in higher dimensions

$$
-\Delta u+V(x) u-\lambda u=f(x), \quad x \in \mathbb{R}^{d},
$$

where $V$ is a smooth real-valued function, periodic with respect to the integer lattice $\mathbb{Z}^{d}$, $f \in C_{\mathrm{c}}^{\infty}\left(\mathbb{R}^{d}\right)$.

The corresponding Schrödinger operator is

$$
H=-\Delta+V(x) \quad \text { on } \quad L^{2}\left(\mathbb{R}^{d}\right)
$$

Recall from Chapter 2 that the fundamental region $W=[0,1]^{d}$ and the Brillouin zone $B=[-\pi, \pi]^{d}$. Using the direct integal, we have 


$$
\mathcal{U} H \mathcal{U}^{-1}=\int_{B}^{\oplus} H(k) \frac{d k}{(2 \pi)^{d}}
$$

where $H(k)$ is formally the same as $H$ but acts on $k$-quasi-periodic functions on $W$. The operator $H(k)$ has compact resolvent. This implies that there are sequences of eigenvalues $\lambda_{j}(k), j \in \mathbb{N}$ called the band functions and the corresponding eigenfunctions $\psi_{j}(x, k), j \in \mathbb{N}$ called Bloch waves.

Here, we only consider the case spectral parameter is regular in the sense of Definition 4.4. If we add a small absorption $i \epsilon, \epsilon \neq 0$, then (4.3) becomes

$$
-\Delta u+V(x) u-(\lambda+i \epsilon) u=f(x), \quad x \in \mathbb{R}^{d} .
$$

From spectral theorem, (4.5) has a unique solution $u_{\epsilon} \in L^{2}\left(\mathbb{R}^{d}\right)$. From the limiting absorption principle, $u_{\epsilon}$ tends to solution $u$ of the Schrödinger equation without absorption in suitable sense as $\epsilon \rightarrow \pm 0$. We want to characterize this solution.

We will adapt method in [Rad15] and use elliptic regularity to get a smooth solution.

Theorem 4.6 ([RS75, Theorem IX.26] [Fol95, Theorem 6.33], elliptic regularity). Let $u$ be a distribution solution of equation $(-\Delta+V) u=\lambda u$ where $V$ is a measurable function and $\lambda$ is a complex number. If $V$ is a smooth function in open region $\Omega$, then $u$ is also smooth in that region.

Recall Floquet-Bloch-Gelfand transform

$$
\mathcal{U} f(x)=\sum_{n \in \mathbb{Z}^{d}} e^{i n \cdot k} f(x-n)
$$

The inverse has the formulae

$$
\mathcal{U}^{-1} g(x+n)=\frac{1}{(2 \pi)^{d}} \int_{B} e^{i k \cdot n} g(x, k) d k .
$$

Using the following theorem, it is easy to derive an integral formula for the solution of (4.5) (for more details see [Plu11]).

Proposition 4.7 ([Plu11], completeness of Bloch waves). For $g \in L^{2}\left(\mathbb{R}^{d}\right)$ and $j \in \mathbb{N}$, define

$$
g_{j}(x):=\frac{1}{(2 \pi)^{d}} \sum_{s=1}^{j} \int_{B}\left\langle\mathcal{U} g(\cdot, k), \psi_{s}(\cdot, k)\right\rangle_{L^{2}(W)} \psi_{s}(x, k) d k, \quad x \in \mathbb{R}^{d} .
$$

Then, $g_{j} \rightarrow g$ as $j \rightarrow \infty$ in $L^{2}\left(\mathbb{R}^{d}\right)$. 
Theorem 4.8 ([Plu11, Theorem 3.6.2]). For $\epsilon \neq 0$,

$$
u_{\epsilon}(x)=(H-(\lambda+i \epsilon))^{-1} f(x)=\frac{1}{(2 \pi)^{d}} \sum_{j \in \mathbb{N}} \int_{B} \frac{\left\langle\mathcal{U} f(., k), \psi_{j}(., k)\right\rangle_{L^{2}(W)}}{\lambda_{j}(k)-(\lambda+i \epsilon)} \psi_{j}(x, k) d k .
$$

For simplicity, from now on we denote the inner product $\langle\cdot, \cdot\rangle_{L^{2}(W)}$ by $\langle\cdot, \cdot\rangle$.

Remark 4.9. In [Plu11], the author gave a proof by using Proposition 4.7 and some $L^{2}(W)$ estimates. Here, we give a simple proof by using the direct integral and spectral theory. The proof follows the idea in [MT03, Proposition 2.3].

Proof of Theorem 4.8, Recall the direct integral of the operator $H$ from Theorem 2.29

$$
\mathcal{U} H \mathcal{U}^{-1}=\int_{B}^{\oplus} H(k) \frac{d k}{(2 \pi)^{d}}
$$

where $H(k)$ is the operator $-\Delta+V(x)$ on $W$ with the $k$-quasi-periodic boundary conditions. Therefore,

$$
\begin{aligned}
K:=\mathcal{U}(H-\lambda-i \epsilon) \mathcal{U}^{-1} & =\mathcal{U} H \mathcal{U}^{-1}-(\lambda+i \epsilon) I \\
& =\int_{B}^{\oplus} H(k) \frac{d k}{(2 \pi)^{d}}-(\lambda+i \epsilon) I \\
& =\int_{B}^{\oplus}(H(k)-\lambda-i \epsilon) \frac{d k}{(2 \pi)^{d}} .
\end{aligned}
$$

For $\epsilon \neq 0,(H(k)-\lambda-i \epsilon)$ is invertiable and its inverse is analytic from $B$ to $\mathscr{L}\left(L^{2}(W)\right)$. By Theorem 2.21, operator

$$
P:=\int_{B}^{\oplus}(H(k)-\lambda-i \epsilon)^{-1} \frac{d k}{(2 \pi)^{d}}
$$

is a bounded operator on $L^{2}\left(\mathbb{R}^{d}\right)=\int_{B}^{\oplus} L^{2}(W) d k$. We will prove that $P$ is the inverse of $K$.

Indeed, for any $\phi(x) \in L^{2}\left(\mathbb{R}^{d}\right)$, put $\varphi=P(\phi)$. Then,

$$
\varphi(k)=(H(k)-\lambda-i \epsilon)^{-1} \phi(k) .
$$

This implies $(H(k)-\lambda-i \epsilon) \varphi(k)=\phi(k)$. That means $\varphi(k) \in D(H(k))$ and $\varphi \in D(K)$. Hence, $K P(\phi)=\phi$, i.e., $P$ is a right inverse of $K$.

Moreover, for any $\varphi \in D(K)=H^{2}\left(\mathbb{R}^{d}\right)$, put $\phi=K \varphi$. Then,

$$
\phi(k)=(H(k)-\lambda-i \epsilon) \varphi(k)
$$


Because $H(k)$ is an operator on $L^{2}(W)$ with the domain $H_{k}^{2}(W)$, it implies $\phi(k) \in L^{2}(W)$. Therefore $\phi \in L^{2}\left(\mathbb{R}^{d}\right)$ and $P K(\varphi)=\varphi$. That means $P$ is a left inverse of $K$. Thus, $P$ is the inverse of $K$.

We have

$$
H-\lambda-i \epsilon=\mathcal{U}^{-1} K U
$$

Take the inverse both sides of (??), it implies

$$
(H-\lambda-i \epsilon)^{-1}=\mathcal{U}^{-1} L U
$$

Applying both sides of (4.9) to $f$, we get

$$
u_{\epsilon}(x)=\mathcal{U}^{-1} L \mathcal{U} f(x)=\mathcal{U}^{-1}\left(\int_{B}^{\oplus}(H(k)-\lambda-i \epsilon)^{-1} \frac{d k}{(2 \pi)^{d}}\right) \mathcal{U} f(x)
$$

Moreover, $H(k)$ has sequences of eigenvalues $\lambda_{j}(k)$ and the corresponding eigenfunctions $\psi_{j}(x, k), j \in \mathbb{N}$, called Bloch waves, which form an orthonormal basis of $L^{2}(W)$. It implies

$$
\begin{aligned}
(H(k)-\lambda-i \epsilon)^{-1} \mathcal{U} f(x, k) & =(H(k)-\lambda-i \epsilon)^{-1} \sum_{j \in \mathbb{N}}\left\langle\mathcal{U} f(., k), \psi_{j}(., k)\right\rangle \psi_{j}(x, k) \\
& =\sum_{j \in \mathbb{N}}\left\langle\mathcal{U} f(., k), \psi_{j}(., k)\right\rangle(H(k)-\lambda-i \epsilon)^{-1} \psi_{j}(x, k) \\
& =\sum \frac{\left\langle\mathcal{U} f(., k), \psi_{j}(., k)\right\rangle}{\lambda_{j}(k)-(\lambda+i \epsilon)} \psi_{j}(x, k) .
\end{aligned}
$$

Thus, we obtain (4.7).

Definition 4.10. Define a set

$$
J(\lambda)=\left\{j \in \mathbb{N}: \exists k \in B, \lambda_{j}(k)=\lambda\right\},
$$

and level sets of $\lambda$

$$
\Gamma_{j}(\lambda)=\left\{k \in[-\pi, \pi]^{d}: \lambda_{j}(k)=\lambda\right\} \quad \text { for } \quad j \in J(\lambda)
$$

For $\omega \in \mathbb{S}^{d-1}$, define

$$
L_{j}(\omega)=\left\{k_{\omega} \in \Gamma_{j}(\lambda): \omega=\nabla \lambda_{j}\left(k_{\omega}\right) /\left|\nabla \lambda_{j}\left(k_{\omega}\right)\right|\right\} \quad \text { for } \quad j \in J(\lambda) .
$$

Remark 4.11. The set $L_{j}(\omega)$ play an important role in the asymptotic expansion of the outgoing solution. We will see that all points in this set are stationary points. 
Lemma 4.12. The set $J(\lambda)$ is finite.

Proof. For every $k \in B$, the band functions $\lambda_{j}(k)$ tend to infinity as $j \rightarrow \infty$. This implies that the set $J(\lambda)$ is finite.

First, we have the following proposition which describes the limit of components in (4.7) when $j \notin J(\lambda)$ :

Proposition 4.13. For $j \notin J(\lambda)$,

$$
U_{j, \epsilon}(x)=\frac{1}{(2 \pi)^{d}} \int_{B} \frac{\left\langle\mathcal{U} f(., k), \psi_{j}(., k)\right\rangle}{\lambda_{j}(k)-\lambda-i \epsilon} \psi_{j}(x, k) d k
$$

converges to $U_{j}(x)$ in $L^{2}\left(\mathbb{R}^{d}\right)$ as $\epsilon \rightarrow 0$, where

$$
U_{j}(x)=\frac{1}{(2 \pi)^{d}} \int_{B} \frac{\left\langle\mathcal{U} f(., k), \psi_{j}(., k)\right\rangle}{\lambda_{j}(k)-\lambda} \psi_{j}(x, k) d k .
$$

Proof. Floquet-Bloch-Gelfand transform is a unitary operator from $L^{2}\left(\mathbb{R}^{d}\right)$ to $L^{2}\left(B ; L^{2}(W)\right)$ (see Theorem 2.9). Take $L^{2}$-norm of difference $U_{j, \epsilon}(x)-U_{j}(x)$ and use above property, we get

$$
\begin{aligned}
\left\|U_{j, \epsilon}-U_{j}\right\|_{L^{2}\left(\mathbb{R}^{d}\right)}^{2} & =\left\|\frac{1}{(2 \pi)^{d}} \int_{B}\left(\frac{\left\langle\mathcal{U} f(., k), \psi_{j}(., k)\right\rangle}{\lambda_{j}(k)-\lambda-i \epsilon}-\frac{\left\langle\mathcal{U} f(., k), \psi_{j}(., k)\right\rangle}{\lambda_{j}(k)-\lambda}\right) \psi_{j}(x, k) d k\right\|_{L^{2}\left(\mathbb{R}^{d}\right)}^{2} \\
& =\left\|\mathcal{U}^{-1}\left\{\left(\frac{\left\langle\mathcal{U} f(., k), \psi_{j}(., k)\right\rangle}{\lambda_{j}(k)-\lambda-i \epsilon}-\frac{\left\langle\mathcal{U} f(., k), \psi_{j}(., k)\right\rangle}{\lambda_{j}(k)-\lambda}\right) \psi_{j}(x, k)\right\}\right\|_{L^{2}\left(\mathbb{R}^{d}\right)}^{2} \\
& =\left\|\left(\frac{\left\langle\mathcal{U} f(., k), \psi_{j}(., k)\right\rangle}{\lambda_{j}(k)-\lambda-i \epsilon}-\frac{\left\langle\mathcal{U} f(., k), \psi_{j}(., k)\right\rangle}{\lambda_{j}(k)-\lambda}\right) \psi_{j}(x, k)\right\|_{L^{2}\left(B ; L^{2}(W)\right)}^{2} \\
& \left.=\int_{B} \mid \| \frac{\left\langle\mathcal{U} f(., k), \psi_{j}(., k)\right\rangle}{\lambda_{j}(k)-\lambda-i \epsilon}-\frac{\left\langle\mathcal{U} f(., k), \psi_{j}(., k)\right\rangle}{\lambda_{j}(k)-\lambda}\right) \psi_{j}(x, k) \|_{L^{2}(W)}^{2} d k \\
& =\int_{B} \frac{\left\langle\mathcal{U} f(., k), \psi_{j}(., k)\right\rangle}{\lambda_{j}(k)-\lambda-i \epsilon}-\left.\frac{\left\langle\mathcal{U} f(., k), \psi_{j}(., k)\right\rangle}{\lambda_{j}(k)-\lambda}\right|^{2}\left\|\psi_{j}(., k)\right\|_{L^{2}(W)}^{2} d k \\
& =\int_{B}\left|\frac{\left\langle\mathcal{U} f(., k), \psi_{j}(., k)\right\rangle}{\lambda_{j}(k)-\lambda-i \epsilon}-\frac{\left\langle\mathcal{U} f(., k), \psi_{j}(., k)\right\rangle}{\lambda_{j}(k)-\lambda}\right|^{2} d k .
\end{aligned}
$$

The last equality uses the complete orthonormal basis $\psi_{j}(., k), j \in \mathbb{N}$ in $L^{2}(W)$ for any fixed $k \in B$. 
Because $j \notin J(\lambda)$, there exists a constant $C$ such that $\left|\lambda_{j}(k)-\lambda-i \epsilon\right|^{-1}$ and $\left|\lambda_{j}(k)-\lambda\right|^{-1}$ are smaller than $C$. Combine this with Cauchy-Schwarz inequality, it implies

$$
\left\|U_{j, \epsilon}-U_{j}\right\|_{L^{2}\left(\mathbb{R}^{d}\right)}^{2} \leq \epsilon^{2} C^{4} \int_{B}\|\mathcal{U} f(., k)\|_{L^{2}(W)} d k=\epsilon^{2} C^{4}\|f\|_{L^{2}\left(\mathbb{R}^{d}\right)}
$$

Therefore, $U_{j, \epsilon} \rightarrow U_{j}$ in $L^{2}\left(\mathbb{R}^{d}\right)$ as $\epsilon \rightarrow 0$. A consequence of this convergence is $U_{j}(x) \in L^{2}\left(\mathbb{R}^{d}\right)$.

Remark 4.14. Because the spectrum of $H$ is purely absolutely continuous (see Chapter 2), we only need to get $L^{2}\left(\mathbb{R}^{d}\right)$ reminder terms in the asymptotic expansion. Proposition 4.13 says that the main contribution to the asymptotic expansion of outgoing solution comes from the integrals in (4.7) for $j \in J(\lambda)$.

Proposition 4.15. For $\epsilon \neq 0$,

$$
u_{\epsilon}(x)=(H-(\lambda+i \epsilon))^{-1} f=\mathcal{U}^{-1} R(k, \lambda+i \epsilon) \mathcal{U} f
$$

where $R(k, \lambda+i \epsilon)=(H(k)-\lambda-i \epsilon)^{-1}$.

Proof. This proposition is a consequence of Theorem 4.8, We obtain the formula

$$
u_{\epsilon}(x)=\mathcal{U}^{-1} L \mathcal{U} f(x)=\mathcal{U}^{-1}\left(\int_{B}^{\oplus}(H(k)-\lambda-i \epsilon)^{-1} \frac{d k}{(2 \pi)^{d}}\right) \mathcal{U} f(x)
$$

Using the direct integral property, it implies

$$
\begin{aligned}
\left(\int_{B}^{\oplus}(H(k)-\lambda-i \epsilon)^{-1} \frac{d k}{(2 \pi)^{d}}\right) \mathcal{U} f(x, k) & =(H(k)-\lambda-i \epsilon)^{-1} \mathcal{U} f(x, k) \\
& =(R(k, \lambda+i \epsilon)) \mathcal{U} f(x, k) .
\end{aligned}
$$

Therefore, we get (4.12).

Lemma 4.16. For each $j \in J(\lambda)$, there exists a small neighborhood $D_{j}(\lambda)$ of $\Gamma_{j}(\lambda)$ and $\delta>0$ such that for $k \in D_{j}(\lambda)$,

$$
\sigma(H(k)) \cap\{z \in \mathbb{C}:|z-\lambda|<2 \delta\}=\left\{\lambda_{j}(k)\right\}
$$

Remark 4.17. The intersection (4.13) contains only one eigenvalue $\lambda_{j}(k)$. But it could happen that it is degenerated, i.e., the multiplicity of eigenvalue is more than one. 
Proof of Lemma 4.16. Firstly, note that $\lambda_{j}(k)$ is an isolated eigenvalue of finite multiplicity. Without loss of generality, we can assume $\lambda_{j}(k)$ is a non-degenerated eigenvalue. Then, choose $\delta>0$ such that $2 \delta<\min \left\{\lambda_{j+1}(k)-\lambda_{j}(k), \lambda_{j}(k)-\lambda_{j-1}(k)\right\}$.

Moreover, $\lambda_{j}(k)$ is continuous. Therefore, there exists a small neighborhood $D_{j}(\lambda)$ of $\Gamma_{j}(\lambda)$ such that $\left|\lambda_{j}(k)-\lambda\right|<\delta$ for all $k \in D_{j}(\lambda)$.

It is easy to see that for $k \in D_{j}(\lambda)$, (4.13) satisfies.

Proposition 4.18. For $(k, z) \in \bigcup_{j \in J(\lambda)} D_{j}(\lambda) \times\{z \in \mathbb{C}:|z-\lambda|<\delta\}$, where $z \neq \lambda_{j}(k)$ for all $j \in J(\lambda)$

$$
R(k, z)=\sum_{j \in J(\lambda)}\left(\lambda_{j}(k)-z\right)^{-1} P_{j}(k)+Q(k, z)
$$

Here, $P_{j}(k)$ are projections onto eigenspaces corresponding to eigenvalues $\lambda_{j}(k), Q(k, z)$ is analytic with respect to $k \in D_{j}(\lambda)$ for $\{z \in \mathbb{C}:|z-\lambda|<\delta\}$.

Proof. The resolvent $R(k, z)$ is a meromorphic function of $z$ near $\lambda$ with poles at $\lambda_{j}(k), j \in$ $J(\lambda)$.

It is enough to show the order of the pole $\lambda_{j}(k)$ is one. Indeed, since $H(k)$ is selfadjoint, therefore $H(k)-z$ is normal for all $z \in \mathbb{C}$. This implies the above statement (see [Kat95, Chapter 2, Theorem 1.10]).

Remark 4.19. Even if $\lambda_{j}(k)$ is not simple, we still have (4.14).

Remark 4.20. The projection $P_{j}(k)$ are not analytic in general. It is fulfilled if $\lambda_{j}(k)$ are simple. If $\lambda_{j}(k)$ is an eigenvalue of multiplicity $m>1$, then only total projection $P=\sum_{l \leq m} P_{j, l}(k)$ is analytic (see [Kat95]). In order to establish a radiation condition, we need to assume the local smoothness of the eigenvalues $\lambda_{j}(k)$ and the eigen-projections $P_{j}(k)$ near the level sets $\Gamma_{j}(\lambda), j \in J(\lambda)$.

The main contribution to the asymptotic expansion of the solution comes from integrals near the level set $\Gamma_{j}(\lambda)$. Therefore, we need to localize these integrals near the level sets by using partition of unity.

Proposition 4.21 ([Hör83], partition of unity). Let $X_{1}, \ldots, X_{m}$ be open sets in $\mathbb{R}^{d}$ and $K$ a compact subset of $\bigcup_{1}^{m} X_{j}$. Then, one can find $\rho_{j} \in C_{\mathrm{c}}^{\infty}\left(X_{j}\right)$ so that $\rho_{j} \geq 0$ and $\sum_{1}^{m} \rho_{j} \leq 1$ with equality in a neighborhood of $K$.

The reminder terms can be estimated by using the relation between the decreasing of a function and the smoothness of its Floquet transform. This relation is similar to the classical result for the Fourier transform. 
Theorem 4.22 (Paley-Wiener theorem). If $f$ is a smooth function with compact support in $\mathbb{R}^{d}$, then its Fourier transform $F(z)$ is an entire function in $\mathbb{C}^{d}$ and for any $N>0$, there exist $C_{N}, R>0$ such that

$$
|F(z)| \leq C_{N}(1+|z|)^{-N} e^{R|\operatorname{Im} z|}
$$

Conversely, if an entire function $F(z)$ satisfies the condition (4.15), then it is the Fourier transform of a smooth function with compact support in $\mathbb{R}^{d}$.

Remark 4.23. The Floquet-Bloch-Gelfand transform $\mathcal{U} f(x, k)$ of function $f \in L^{2}\left(\mathbb{R}^{d}\right)$ is periodic in $k$ with respect to the dual lattice $\Gamma^{*}$ and quasi-periodic in $x$ with quasimomentum $k$ (see [Kuc93]). Therefore, there is no decaying of the Floquet-BlochGelfand transform of a smooth function with compact support as (4.15). But it is easy to see that $\mathcal{U} f(x, k)$ is an analytic function in $k$ for $f \in C_{\mathrm{c}}^{\infty}\left(\mathbb{R}^{d}\right)$. Moreover, one has the following theorem:

Theorem 4.24 ([Kuc16, KKR17]). Let $s \geq 0$ and $g(x, k)$ be periodic in $k$ with respect to the dual lattice $\Gamma^{*}$ and quasi-periodic in $x$ with quasi-momentum $k$ (see Proposition 2.10). Then:

i) If $g(x, k)$ is smooth in $k \in \mathbb{R}^{d}$ with values in $H_{k}^{s}(W)$, then $f:=\mathcal{U}^{-1} g \in H^{s}\left(\mathbb{R}^{d}\right)$ and for any $N>0, \gamma \in \Gamma$,

$$
\|f\|_{H^{s}(W+\gamma)} \leq C_{N}|\gamma|^{-N}
$$

In particular, by Sobolev embedding theorem (see e.g. [Eva10, GT01]), if $s>d / 2$, then the pointwise estimation holds

$$
|f(x)| \leq C_{N}(1+|x|)^{-N}
$$

ii) If $g(x, k)$ is analytic in $k \in \mathbb{R}^{d}$ with values in $H_{k}^{s}(W)$, then $f:=\mathcal{U}^{-1} g \in H^{s}\left(\mathbb{R}^{d}\right)$. Moreover, there exist positive constants $C_{1}, C_{2}$ such that for any $\gamma \in \Gamma$,

$$
\|f\|_{H^{s}(W+\gamma)} \leq C_{1} e^{-C_{2}|\gamma|} .
$$

In particular, by Sobolev embedding theorem (see e.g. [Eva10, GT01]), if $s>d / 2$, then the pointwise estimation holds

$$
|f(x)| \leq C_{1} e^{-C_{2}|x|} .
$$


Consider a finite open cover of $B$ which consists of $D_{p}(\lambda), p \in J(\lambda)$ and $U_{l}, l \in I(\lambda)$, where $I(\lambda)$ is a finite set such that $U_{l}, l \in I(\lambda)$ contain no point on the level sets $\Gamma_{p}(\lambda)$ for all $p \in J(\lambda)$. Let $\rho_{p}(k), p \in J(\lambda), \chi_{l}(k), l \in I(\lambda)$ be subordinate to the open cover $D_{p}(\lambda), p \in J(\lambda)$ and $U_{l}, l \in I(\lambda)$, i.e., $\operatorname{supp} \rho_{p} \subset D_{j}(\lambda)$, supp $\chi_{l} \subset U_{l}$ and $\sum_{p \in J(\lambda)} \rho_{p}(k)+$ $\sum_{l \in I(\lambda)} \chi_{l}(k)=1$. Then, using (4.14) we get

$$
\begin{aligned}
u_{\epsilon}(x)= & \sum_{p \in J(\lambda)} \mathcal{U}^{-1} \rho_{p}(k) R(k, \lambda+i \epsilon) \mathcal{U} f+\sum_{l \in I(\lambda)} \mathcal{U}^{-1} \chi_{l}(k) R(k, \lambda+i \epsilon) \mathcal{U} f \\
= & \sum_{p \in J(\lambda)} \sum_{j \in J(\lambda)} \mathcal{U}^{-1} \rho_{p}(k)\left(\lambda_{j}(k)-\lambda-i \epsilon\right)^{-1} P_{j}(k) \mathcal{U} f+ \\
& +\sum_{p \in J(\lambda)} \mathcal{U}^{-1} \rho_{p}(k) Q(k, \lambda+i \epsilon) \mathcal{U} f+\sum_{l \in I(\lambda)} \mathcal{U}^{-1} \chi_{l}(k) R(k, \lambda+i \epsilon) \mathcal{U} f .
\end{aligned}
$$

Remark 4.25. We will prove that the main contribution to the asymptotic expansion of solution $u=\lim _{\epsilon \rightarrow+0} u_{\epsilon}$ of the Schrödinger equation (4.3) comes from the first part of (4.16). The reminder terms can be estimated by using Theorem 4.24.

Theorem 4.26. As $\epsilon \rightarrow 0$,

$$
\begin{aligned}
F(\lambda+i \epsilon) f(x):= & \sum_{l \in I(\lambda)} \mathcal{U}^{-1} \chi_{l}(k) R(k, \lambda+i \epsilon) \mathcal{U} f \\
& \rightarrow F(\lambda) f(x):=\sum_{l \in I(\lambda)} \mathcal{U}^{-1} \chi_{l}(k) R(k, \lambda) \mathcal{U} f \quad \text { in } \quad L^{2}\left(\mathbb{R}^{d}\right),
\end{aligned}
$$

and for $n \in \mathbb{Z}^{d}, N \in \mathbb{N}$,

$$
F(\lambda) f(x+n)=O\left(|n|^{-N}\right) \quad \text { as } \quad|n| \rightarrow \infty .
$$

Proof. For $k \in U_{l}, l \in I(\lambda)$, we have $\lambda_{j}(k) \neq \lambda$ for all $j \in J(\lambda)$. Therefore, $R(k, \lambda)$ is well-defined for all $k \in U_{l}$. This implies $R(k, \lambda+i \epsilon) \rightarrow R(k, \lambda)$ for all $k \in U_{l}, l \in I(\lambda)$ as $\epsilon \rightarrow 0$ in operator norm, and

$$
F(\lambda+i \epsilon) f(x) \rightarrow F(\lambda) f(x) \quad \text { in } \quad L^{2}\left(\mathbb{R}^{d}\right) \quad \text { as } \quad \epsilon \rightarrow 0
$$

Because $f(x)$ is a smooth function with compact support, this implies $\mathcal{U} f(x, k)$ is analytic in $k \in B$ (see Remark 4.23). Moreover, the resolvent $R(k, \lambda)$ is analytic in $k \in U_{l}, l \in I(\lambda)$. Therefore, $\chi_{l}(k) R(k, \lambda) \mathcal{U} f(x, k)$ is smooth in $k \in U_{l}, l \in I(\lambda)$. This implies the estimate (4.17) (see Theorem 4.24).

Using the same argument, we obtain 
Proposition 4.27. As $\epsilon \rightarrow 0$,

$$
\begin{aligned}
G(\lambda+i \epsilon) f(x):= & \sum_{p \in J(\lambda)} \mathcal{U}^{-1} \rho_{p}(k) Q(k, \lambda+i \epsilon) \mathcal{U} f(x) \\
& \rightarrow G(\lambda) f(x):=\sum_{p \in J(\lambda)} \mathcal{U}^{-1} \rho_{p}(k) Q(k, \lambda) \mathcal{U} f(x) \quad \text { in } \quad L^{2}\left(\mathbb{R}^{d}\right),
\end{aligned}
$$

and for $n \in \mathbb{Z}^{d}$ and $N \in \mathbb{N}$

$$
G(\lambda) f(x+n)=O\left(|n|^{-N}\right) \quad \text { as } \quad|n| \rightarrow \infty .
$$

Proof. From Proposition 4.18, $Q(k, \lambda)$ is well-defined and analytic in $\lambda$ and in $k \in D_{j}(\lambda)$. Therefore, it implies the convergence $G(\lambda+i \epsilon) f(x) \rightarrow G(\lambda) f(x)$ in $L^{2}\left(\mathbb{R}^{d}\right)$ as $\epsilon \rightarrow 0$ and the estimate (4.18) (see Theorem 4.24).

Taking the limit $u_{\epsilon}$ as $\epsilon \rightarrow+0$, we get

$$
u(x)=\lim _{\epsilon \rightarrow+0} u_{\epsilon}(x)=F(\lambda) f(x)+G(\lambda) f(x)+\sum_{p \in J(\lambda)} K_{p}(x)
$$

where

$$
\begin{aligned}
K_{p}(x) & =\lim _{\epsilon \rightarrow+0} \mathcal{U}^{-1} \rho_{p}(k) \sum_{j \in J(\lambda)} \frac{P_{j}(k)}{\lambda_{j}(k)-\lambda-i \epsilon} \mathcal{U} f \\
& =\lim _{\epsilon \rightarrow+0} \frac{1}{(2 \pi)^{d}} \int_{B} \rho_{p}(k) \sum_{j \in J(\lambda)} \frac{\left\langle\mathcal{U} f(., k), \psi_{j}(., k)\right\rangle}{\lambda_{j}(k)-(\lambda+i \epsilon)} \psi_{j}(x, k) d k .
\end{aligned}
$$

To analyze the integral (4.19), let $\omega \in \mathbb{S}^{d-1}$. Take functions $\phi_{m}(k) \in C_{\mathrm{c}}^{\infty}\left(D_{p}(\lambda)\right), m=$ $1,2,3$, such that:

i) $\phi_{1}(k)=1$ near $L_{p}(\omega)$ and $\omega \cdot \nabla \lambda_{p}>0$ on the support of $\phi_{1}$,

ii) $\phi_{2}(k)=1$ near $L_{p}(-\omega)$ and $\omega \cdot \nabla \lambda_{p}<0$ on the support of $\phi_{2}$,

iii) $\phi_{3}(k)=1$ near $\left\{k \in \Gamma_{p}(\lambda): \omega \cdot \nabla \lambda_{p}=0\right\}$,

iv) $\sum_{m=1}^{3} \phi_{l}=1$ near $\Gamma_{p}(\lambda)$.

Therefore,

$$
K_{p}(x)=K_{1 p}(x)+K_{2 p}(x)+K_{3 p}(x),
$$

where

$$
K_{m p}(x)=\lim _{\epsilon \rightarrow+0} \frac{1}{(2 \pi)^{d}} \int_{B} \phi_{m}(k) \rho_{p}(k) \sum_{j \in J(\lambda)} \frac{\left\langle\mathcal{U} f(., k), \psi_{j}(., k)\right\rangle}{\lambda_{j}(k)-(\lambda+i \epsilon)} \psi_{j}(x, k) d k, \quad m=1,2,3 .
$$


First, consider $K_{1 p}$. We choose $\left\{e_{2}^{\prime}, \ldots, e_{d}^{\prime}\right\} \subset \mathbb{R}^{d}$ such that $\left\{\omega, e_{2}^{\prime}, \ldots, e_{d}^{\prime}\right\}$ is an orthonormal basis of $\mathbb{R}^{d}$, and use the coordinate $\left(k_{1}, k^{\prime}\right)$ such that

$$
k=k_{1} \omega+k^{\prime} e^{\prime} .
$$

Changing of variables from $\left(k_{1}, k^{\prime}\right)$ to $\left(\xi_{1}, \xi^{\prime}\right)$ such that $\xi_{1}=\lambda_{j}(k)-\lambda, \xi^{\prime}=k^{\prime}$, we obtain for $p \in J(\lambda), j \in J(\lambda)$,

$$
\begin{aligned}
& K_{1 p j \epsilon}(x):= \frac{1}{(2 \pi)^{d}} \int_{B} \phi_{1}(k) \rho_{p}(k) \frac{\left\langle\mathcal{U} f(., k), \psi_{j}(., k)\right\rangle}{\lambda_{j}(k)-(\lambda+i \epsilon)} \psi_{j}(x, k) d k \\
&=\frac{1}{(2 \pi)^{d}} \int_{B^{\prime}} \phi_{1}\left(k_{1}(\xi) \omega+\xi^{\prime} e^{\prime}\right) \rho_{p}\left(k_{1}(\xi) \omega+\xi^{\prime} e^{\prime}\right) . \\
& \quad \frac{\left.\left\langle\mathcal{U} f\left(., k_{1}(\xi) \omega+\xi^{\prime} e^{\prime}\right), \psi_{j}\left(., k_{1}(\xi) \omega+\xi^{\prime} e^{\prime}\right)\right)\right\rangle}{\xi_{1}-i \epsilon} \psi_{j}\left(x, k_{1}(\xi) \omega+\xi^{\prime} e^{\prime}\right)\left|\frac{\partial k_{1}}{\partial \xi_{1}}\right| d \xi,
\end{aligned}
$$

where

$$
\frac{\partial k_{1}}{\partial \xi_{1}}=\left(\frac{\partial \xi_{1}}{\partial k_{1}}\right)^{-1}=\left(\omega \cdot \nabla \lambda_{j}\left(k_{1}(\xi) \omega+\xi^{\prime} e^{\prime}\right)\right)^{-1}>0 .
$$

Using a basic inequality for $\phi \in C_{\mathrm{c}}^{1}(\mathbb{R})$ and $\epsilon>0$ (see e.g. [MT06])

$$
\mid \int_{\mathbb{R}}\left[\frac{1}{t-i \epsilon}-\left(\text { p.v. } \frac{1}{t}+i \pi \delta(t)\right)\right] \phi(t) d t \mid \leq C \epsilon\|\phi\|_{C^{1}},
$$

and taking the limit as $\epsilon \rightarrow+0$, it implies

$$
\begin{gathered}
K_{1 p j}(x):=\lim _{\epsilon \rightarrow+0} K_{1 p j \epsilon}(x)=\frac{1}{(2 \pi)^{d}} \int_{B^{\prime}} \phi_{1}\left(k_{1}(\xi) \omega+\xi^{\prime} e^{\prime}\right) \rho_{p}\left(k_{1}(\xi) \omega+\xi^{\prime} e^{\prime}\right)\left(\text { p.v. } \frac{1}{\xi_{1}}+i \pi \delta\left(\xi_{1}\right)\right) \\
\frac{\left.\left\langle\mathcal{U} f\left(., k_{1}(\xi) \omega+\xi^{\prime} e^{\prime}\right), \psi_{j}\left(., k_{1}(\xi) \omega+\xi^{\prime} e^{\prime}\right)\right)\right\rangle}{\omega \cdot \nabla \lambda_{j}\left(k_{1}(\xi) \omega+\xi^{\prime} e^{\prime}\right)} \psi_{j}\left(x, k_{1}(\xi) \omega+\xi^{\prime} e^{\prime}\right) d \xi .
\end{gathered}
$$

Remark 4.28. The quantity $\omega \cdot \nabla \lambda_{j}\left(k_{1}(\xi) \omega+\xi^{\prime} e^{\prime}\right)$ is related to the group velocity of wave propagation in periodic media. If it is positive (negative), then the group velocity is positive (negative).

Remark 4.29. By the defintion, all points in $L_{j}(\omega)$ have positive group velocity. These points will play important role in the asymptotic expansion of the solution of the periodic Schrödinger equation.

We want to write the solution of the periodic Schrödinger equation without absorption as oscillatory integral and use the stationary phase method. First, we fix $x \in W$ and introduce a variable $n \in \mathbb{Z}^{d}$. Then, using quasi-periodic property of Bloch waves, it implies

$$
u(x+n)=F(\lambda) f(x+n)+G(\lambda) f(x+n)+\sum_{p \in J(\lambda)} K_{p}(x+n)
$$


where

$$
K_{p}(x+n)=\lim _{\epsilon \rightarrow+0} \frac{1}{(2 \pi)^{d}} \int_{B} \rho_{p}(k) \sum_{j \in J(\lambda)} \frac{\left\langle\mathcal{U} f(., k), \psi_{j}(., k)\right\rangle}{\lambda_{j}(k)-(\lambda+i \epsilon)} \psi_{j}(x, k) e^{i k \cdot n} d k .
$$

Using the same argument as before for (4.21) with $\omega=n /|n| \in \mathbb{S}^{n-1}$, we obtain

$$
\begin{aligned}
K_{1 p j}(x+n)=\frac{1}{(2 \pi)^{d}} & \int_{B^{\prime}} \phi_{1}\left(k_{1}(\xi) \omega+\xi^{\prime} e^{\prime}\right) \rho_{p}\left(k_{1}(\xi) \omega+\xi^{\prime} e^{\prime}\right)\left(\text { p.v. } \frac{1}{\xi_{1}}+i \pi \delta\left(\xi_{1}\right)\right) e^{i\left(n_{1} k_{1}(\xi)+\xi^{\prime} n^{\prime}\right)} \\
& \cdot \frac{\left.\left\langle\mathcal{U} f\left(., k_{1}(\xi) \omega+\xi^{\prime} e^{\prime}\right), \psi_{j}\left(., k_{1}(\xi) \omega+\xi^{\prime} e^{\prime}\right)\right)\right\rangle}{\omega \cdot \nabla \lambda_{j}\left(k_{1}(\xi) \omega+\xi^{\prime} e^{\prime}\right)} \psi_{j}\left(x, k_{1}(\xi) \omega+\xi^{\prime} e^{\prime}\right) d \xi .
\end{aligned}
$$

Next, we use the following estimate:

Lemma 4.30 ([MT06, Lemma 2.4]). Let $g(t) \in C_{\mathrm{c}}^{\infty}(\mathbb{R})$, and $\phi(t)$ be a real-valued smooth function. Assume $\phi^{\prime}(t)>0$ on $\operatorname{supp} g$, then for any $N \in \mathbb{N}$,

$$
\text { p.v. } \int_{-\infty}^{\infty} e^{i r \phi(t)} g(t) \frac{1}{t} d t= \pm i \pi e^{i r \phi(0)} g(0)+O\left(|r|^{-N}\right)
$$

as $r \rightarrow \pm \infty$.

Remark 4.31. We would like to apply the stationary phase method for integral $K_{1 p j}$. Therefore, we need regularity on the band functions $\lambda_{j}(k)$ and Bloch waves $\psi_{j}(x, k), j \in$ $J(\lambda)$.

Remark 4.32. In periodic waveguide [FJ16], it is allowed band crossing. The strong result on analytic perturbation theory of Rellich [Re169] gives raise the reordering band functions which are real analytic. Here, we also accept band crossing with an assumption on local smoothness of the band functions $\lambda_{j}(k)$ and Bloch waves $\psi_{j}(x, k)$ near the level sets $\Gamma_{j}(\lambda), j \in J(\lambda)$.

Theorem 4.33. Assuming that there exists a system of the band functions $\lambda_{j}(k)$ and Bloch waves $\psi_{j}(k, x)$ which is locally smooth near the level sets $\Gamma_{j}(\lambda), j \in J(\lambda)$, the number of points in $L_{j}(\omega)$ is finite for all $j \in J(\lambda), \omega \in \mathbb{S}^{d-1}$, and Gauss-Kronecker curvature of $\Gamma_{j}(\lambda)$ oriented by $-\nabla \lambda_{j}(k) /\left|\nabla \lambda_{j}(k)\right|$ at any point $k_{\omega} \in L_{j}(\omega), j \in J(\lambda)$ is non-zero. Then, there is an asymptotic expansion

$$
\begin{aligned}
& K_{1 p}(x+n)=\frac{i}{(2 \pi)^{(d-1) / 2}} \sum_{j \in J(\lambda)} \sum_{k_{\omega} \in L_{j}(\omega)} \rho_{p}\left(k_{\omega}\right) \frac{A_{j}(x, \lambda, \omega)}{|n|^{(d-1) / 2}} e^{i n \mid k_{\omega} \cdot \omega}+ \\
&+O\left(\frac{1}{|n|^{(d+1) / 2}}\right) \quad \text { as }|n| \rightarrow \infty .
\end{aligned}
$$

Here, 
- $\omega=\frac{n}{|n|}$,

- $A_{j}\left(x, \lambda, k_{\omega}\right)=\frac{\left\langle\mathcal{U} f\left(., k_{\omega}\right), \psi_{j}\left(., k_{\omega}\right)\right\rangle e^{\frac{-i \pi}{4} \operatorname{sgn}\left(e_{l}^{\prime} \cdot \operatorname{Hess} \lambda_{j}\left(k_{\omega}\right) e_{m}^{\prime}\right)_{l, m=2}^{d}}}{\left|\nabla \lambda_{j}\left(k_{\omega}\right)\right| \sqrt{\left|K_{j}\left(k_{\omega}\right)\right|}} \psi_{j}\left(x, k_{\omega}\right)$,

where $\left(\omega, e_{2}^{\prime}, \ldots, e_{d}^{\prime}\right)$ is an orthonormal basic of $\mathbb{R}^{d}, K_{j}\left(k_{\omega}\right)$ is the Gauss-Kronecker curvature of $\Gamma_{j}(\lambda)$ at $k_{\omega}$,

- $L_{j}(\omega)=\left\{k_{\omega} \in \Gamma_{j}(\lambda): \omega=\nabla \lambda_{j}\left(k_{\omega}\right) /\left|\nabla \lambda_{j}\left(k_{\omega}\right)\right|\right\} \quad$ for $\quad j \in J(\lambda)$.

Proof. First, note that $\omega \cdot \nabla \lambda_{j}(k)>0$ on the support of $\phi_{1}$ and $n \cdot k=|n| \omega \cdot k=|n| k_{1}(\xi)$. It implies

$$
\frac{\partial k_{1}}{\partial \xi_{1}}=\left(\omega \cdot \nabla \lambda_{j}(k)\right)^{-1}>0 \quad \text { on the support of } \phi_{1} .
$$

Next, applying Lemma 4.30 to $K_{1 p j}$ with respect to variable $\xi_{1}$, we obtain for $|n| \rightarrow \infty$

$$
\begin{aligned}
K_{1 p j}(x+n)=\frac{i}{(2 \pi)^{d-1}} & \int_{C} \phi_{1}\left(k_{1}\left(0, \xi^{\prime}\right) \omega+\xi^{\prime} e^{\prime}\right) \rho_{p}\left(k_{1}\left(0, \xi^{\prime}\right) \omega+\xi^{\prime} e^{\prime}\right) e^{i n \mid k_{1}\left(0, \xi^{\prime}\right)} \\
& \cdot \frac{\left.\left\langle\mathcal{U} f\left(., k_{1}\left(0, \xi^{\prime}\right) \omega+\xi^{\prime} e^{\prime}\right), \psi_{j}\left(., k_{1}\left(0, \xi^{\prime}\right) \omega+\xi^{\prime} e^{\prime}\right)\right)\right\rangle}{\omega \cdot \nabla \lambda_{j}\left(k_{1}\left(0, \xi^{\prime}\right)+\xi^{\prime} e^{\prime}\right)} \\
& \cdot \psi_{j}\left(x, k_{1}\left(0, \xi^{\prime}\right) \omega+\xi^{\prime} e^{\prime}\right) d \xi^{\prime}+O\left(1 /|n|^{-N}\right)
\end{aligned}
$$

We apply the stationary phase method (see Appendix A.3) to the integral (4.24) with respect to variable $\xi^{\prime}$. The stationary points satisfy the equation $\partial_{\xi^{\prime}} k_{1}\left(0, \xi^{\prime}\right)=0$ and $\lambda_{j}\left(k_{1}\left(0, \xi^{\prime}\right) \omega+\xi^{\prime} e^{\prime}\right)=\lambda$ if and only if $\omega$ is the direction of $\nabla \lambda_{j}\left(k_{1}\left(0, \xi^{\prime}\right) \omega+\xi^{\prime} e^{\prime}\right)$. The set of all stationary points is $L_{j}(\omega)=\left\{k_{\omega} \in \Gamma_{j}(\lambda): \omega=\nabla \lambda_{j}\left(k_{\omega}\right) /\left|\nabla \lambda_{j}\left(k_{\omega}\right)\right|\right\}$. By the definition, we have $\phi_{1}\left(k_{\omega}\right)=1$ for all $k_{\omega} \in L_{j}(\lambda), j \in J(\lambda)$. It implies for $|n| \rightarrow \infty$

$$
\begin{aligned}
K_{1 p j}(x+n) & =\frac{i}{(2 \pi)^{d-1}} \frac{(2 \pi)^{(d-1) / 2}}{|n|^{(d-1) / 2}} \sum_{k_{\omega} \in L_{j}(\omega)} \rho_{p}\left(k_{\omega}\right) \frac{e^{\frac{i \pi}{4} \operatorname{sgn} \operatorname{Hess} k_{1}\left(0, \xi_{\omega}^{\prime}\right)}}{\left|\nabla \lambda_{j}\left(k_{\omega}\right)\right|\left|\operatorname{det} \operatorname{Hess} k_{1}\left(0, \xi_{\omega}^{\prime}\right)\right|^{1 / 2}} \\
& \cdot\left\langle\mathcal{U} f\left(., k_{\omega}\right), \psi_{j}\left(., k_{\omega}\right)\right\rangle \psi_{j}\left(x, k_{\omega}\right) e^{\left(i|n| k_{\omega} \cdot \omega\right)}+O\left(\frac{1}{|n|^{(d+1) / 2}}\right)
\end{aligned}
$$

Lemma 4.34. For $2 \leq l, m \leq d$, we have

$$
\begin{aligned}
\left(\operatorname{Hess} k_{1}\left(0, \xi_{\omega}^{\prime}\right)\right)_{l m} & =-\left|\nabla \lambda_{j}\left(k_{\omega}\right)\right|^{-1}\left(e_{l}^{\prime} \cdot \operatorname{Hess} \lambda_{j}\left(k_{\omega}\right) e_{m}^{\prime}\right), \\
\left|\operatorname{det}\left(\operatorname{Hess} k_{1}\left(0, \xi_{\omega}^{\prime}\right)\right)\right| & =\left|\nabla \lambda_{j}\left(k_{\omega}\right)\right|^{-(d-1)}\left|\operatorname{det}\left(e_{l}^{\prime} \cdot \operatorname{Hess} \lambda_{j}\left(k_{\omega}\right) e_{m}^{\prime}\right)_{l, m=2}^{d}\right|,
\end{aligned}
$$

where $\xi_{\omega}^{\prime}$ is a stationary point for the integral (4.24). 
Proof. First, note that $\left\{\omega, e_{2}, \ldots, e_{d}\right\}$ is an orthonormal basis of $\mathbb{R}^{d}$ and

$$
\lambda_{j}\left(k_{1}\left(0, \xi_{\omega}^{\prime}\right) \omega+\xi_{\omega}^{\prime} e^{\prime}\right)=\lambda .
$$

Differentiate (4.27) with respect to $\xi_{l}$, we obtain

$$
\frac{\partial \lambda_{j}\left(k_{1}\left(0, \xi_{\omega}^{\prime}\right) \omega+\xi_{\omega}^{\prime} e^{\prime}\right)}{\partial \xi_{l}}+\frac{\left.\partial \lambda_{j}\left(k_{1}\left(0, \xi_{\omega}^{\prime}\right) \omega+\xi_{\omega}^{\prime} e^{\prime}\right)\right)}{\partial \xi_{1}} \frac{\partial k_{1}\left(0, \xi_{\omega}^{\prime}\right)}{\partial \xi_{l}}=0
$$

Again, differentiate (4.28) with respect to $\xi_{m}$, it implies

$$
\begin{aligned}
\frac{\partial^{2} \lambda_{j}\left(k_{\omega}\right)}{\partial \xi_{l} \partial \xi_{m}} & +\frac{\partial^{2} \lambda_{j}\left(k_{\omega}\right)}{\partial \xi_{l} \partial \xi_{1}} \frac{\partial k_{1}\left(0, \xi_{\omega}^{\prime}\right)}{\partial \xi_{m}}+\frac{\partial \lambda_{j}\left(k_{\omega}\right)}{\partial \xi_{1}} \frac{\partial^{2} k_{1}\left(0, \xi_{\omega}^{\prime}\right)}{\partial \xi_{l} \partial \xi_{m}}+ \\
& +\frac{\partial k_{1}\left(0, \xi_{\omega}^{\prime}\right)}{\partial \xi_{l}}\left(\frac{\partial^{2} \lambda_{j}\left(k_{\omega}\right)}{\partial \xi_{1} \partial \xi_{m}}+\frac{\partial^{2} \lambda_{j}\left(k_{\omega}\right)}{\partial \xi_{1}^{2}} \frac{\partial k_{1}\left(0, \xi_{\omega}^{\prime}\right)}{\partial \xi_{m}}\right)=0 .
\end{aligned}
$$

Moreover,

$$
\frac{\partial \lambda_{j}\left(k_{\omega}\right)}{\partial \xi_{1}}=\omega \cdot \nabla \lambda_{j}\left(k_{\omega}\right)=\left|\nabla \lambda_{j}\left(k_{\omega}\right)\right|
$$

Therefore,

$$
\begin{aligned}
-\left|\nabla \lambda_{j}\left(k_{\omega}\right)\right| \frac{\partial^{2} k_{1}\left(0, \xi_{\omega}^{\prime}\right)}{\partial \xi_{l} \partial \xi_{m}} & =\frac{\partial^{2} \lambda_{j}\left(k_{\omega}\right)}{\partial \xi_{l} \partial \xi_{m}}+\frac{\partial k_{1}\left(0, \xi_{\omega}^{\prime}\right)}{\partial \xi_{l}} \frac{\partial^{2} \lambda_{j}\left(k_{\omega}\right)}{\partial \xi_{1} \partial \xi_{m}}+ \\
& +\frac{\partial k_{1}\left(0, \xi_{\omega}^{\prime}\right)}{\partial \xi_{l}} \frac{\partial k_{1}\left(0, \xi_{\omega}^{\prime}\right)}{\partial \xi_{m}} \frac{\partial^{2} \lambda_{j}\left(k_{\omega}\right)}{\partial \xi_{1}^{2}}+\frac{\partial k_{1}\left(0, \xi_{\omega}^{\prime}\right)}{\partial \xi_{m}} \frac{\partial^{2} \lambda_{j}\left(k_{\omega}\right)}{\partial \xi_{l} \partial \xi_{1}}
\end{aligned}
$$

Since $\xi_{\omega}^{\prime}$ is a stationary point, we have

$$
\frac{\partial k_{1}\left(0, \xi_{\omega}^{\prime}\right)}{\partial \xi_{l}}=0, \quad \forall l=2, \ldots, d
$$

This implies (4.25) and (4.26).

Note that $\left\{e_{2}^{\prime}, \ldots, e_{d}^{\prime}\right\}$ is an orthonormal basis of the tangent space of $\Gamma_{j}(\lambda)$ at $k_{\omega}$. Therefore, the Gauss-Kronecker curvature of $\Gamma_{j}(\lambda)$ at $k_{\omega}$ is

$$
K_{j}\left(k_{\omega}\right)=\frac{\operatorname{det}\left(e_{l}^{\prime} \cdot \operatorname{Hess} \lambda_{j}\left(k_{\omega}\right) e_{m}^{\prime}\right)_{l, m=2}^{d}}{\left|\nabla \lambda_{j}\left(k_{\omega}\right)\right|^{d-1}} .
$$

Combine this with Lemma 4.34 and note that

$$
\operatorname{sgn} \operatorname{Hess} k_{1}\left(0, \xi_{\omega}^{\prime}\right)=-\operatorname{sgn}\left(e_{l}^{\prime} \cdot \operatorname{Hess} \lambda_{j}\left(k_{\omega}\right) e_{m}^{\prime}\right)_{l, m=2}^{d},
$$

we obtain (4.23). 
Remark 4.35. The stationary points live on the level sets $\Gamma_{j}(\lambda), j \in J(\lambda)$ satisfying condition $\omega=\nabla \lambda_{j}\left(k_{1}\left(0, \xi^{\prime}\right) \omega+\xi^{\prime} e^{\prime}\right) /\left|\nabla \lambda_{j}\left(k_{1}\left(0, \xi^{\prime}\right) \omega+\xi^{\prime} e^{\prime}\right)\right|$. That means the group velocity at these points is positive. It is compatible with the classical result for outgoing solution. In a case of incoming solution we will get the condition $\omega=-\nabla \lambda_{j}\left(k_{1}\left(0, \xi^{\prime}\right) \omega+\right.$ $\left.\xi^{\prime} e^{\prime}\right) /\left|\nabla \lambda_{j}\left(k_{1}\left(0, \xi^{\prime}\right) \omega+\xi^{\prime} e^{\prime}\right)\right|$, i.e., the group velocity is negative.

Next, consider the integral

$$
K_{2 p}(x+n)=\lim _{\epsilon \rightarrow+0} \frac{1}{(2 \pi)^{d}} \int_{B} \phi_{2}(k) \rho_{p}(k) \frac{\left\langle\mathcal{U} f(., k), \psi_{j}(., k)\right\rangle}{\lambda_{j}(k)-(\lambda+i \epsilon)} \psi_{j}(x, k) e^{i k \cdot n} d k .
$$

Proposition 4.36. For any positive integer $N$,

$$
K_{2 p}(x+n)=O\left(|n|^{-N}\right)
$$

Proof. Similar to $K_{1 p}(x+n)$, we obtain

$$
\begin{aligned}
K_{2 p j}(x+n)= & \frac{1}{(2 \pi)^{d}} \int_{B^{\prime}} \phi_{2}\left(k_{1}(\xi) \omega+\xi^{\prime} e^{\prime}\right) \rho_{p}\left(k_{1}(\xi) \omega+\xi^{\prime} e^{\prime}\right)\left(\mathrm{p} \cdot \mathrm{v} \cdot \frac{1}{\xi_{1}}+i \pi \delta\left(\xi_{1}\right)\right) e^{i n \mid k_{1}(\xi)} . \\
& \frac{\left.\left\langle\mathcal{U} f\left(., k_{1}(\xi) \omega+\xi^{\prime} e^{\prime}\right), \psi_{j}\left(., k_{1}(\xi) \omega+\xi^{\prime} e^{\prime}\right)\right)\right\rangle}{\omega \cdot \nabla \lambda_{j}\left(k_{1}(\xi) \omega+\xi^{\prime} e^{\prime}\right)} \psi_{j}\left(x, k_{1}(\xi) \omega+\xi^{\prime} e^{\prime}\right) d \xi
\end{aligned}
$$

Apply Lemma 4.30 to $K_{2 p j}(x+n)$ with respect to variable $\xi_{1}$ and note that $\partial k_{1} / \partial \xi_{1}=$ $\omega \cdot \nabla \lambda_{j}(k)<0$ on the support of $\phi_{2}$. Therefore, the factor p.v $\frac{1}{\xi_{1}}$ will cancel the factor $i \pi \delta\left(\xi_{1}\right)$ and we only get the reminder term $O\left(|n|^{-N}\right)$ for any positive integer $N$. It implies the estimate (4.31).

For the last integral,

$$
K_{3 p}(x+n)=\lim _{\epsilon \rightarrow+0} \frac{1}{(2 \pi)^{d}} \int_{B} \phi_{3}(k) \rho_{p}(k) \sum_{j \in J(\lambda)} \frac{\left\langle\mathcal{U} f(., k), \psi_{j}(., k)\right\rangle}{\lambda_{j}(k)-(\lambda+i \epsilon)} \psi_{j}(x, k) e^{i k \cdot n} d k
$$

Using change of variables, it is easy to see that $K_{3 p}(x+n)$ contributes nothing to the asymptotic expansion.

Proposition 4.37. For any positive integer $n \in \mathbb{N}$

$$
K_{3 p}(x+n)=O\left(|n|^{-N}\right)
$$

Remark 4.38. The proof is similar to the proof of Lemma 2.7 in [MT06] with a slight difference. 
Proof of Proposition 4.37 Using partition of unity, it is sufficient to prove for integral over sufficiently small domain.

Let $k_{0} \in \Gamma_{j}(\lambda) \cap \operatorname{supp} \phi_{3}$ and $\chi \in C_{c}^{\infty}$ such that $\chi=1$ near a small neighborhood of $k_{0}$. Consider the integral

$$
K_{3 p j, \epsilon, \chi}(x+n):=\frac{1}{(2 \pi)^{d}} \int_{B} \chi(k) \phi_{3}(k) \rho_{p}(k) \frac{\left\langle\mathcal{U} f(., k), \psi_{j}(., k)\right\rangle}{\lambda_{j}(k)-(\lambda+i \epsilon)} \psi_{j}(x, k) e^{i k \cdot n} d k .
$$

Let $\theta$ be the outward unit normal vector to $\Gamma_{j}(\lambda)$ at $k_{0}$, i.e., $\theta=\nabla \lambda_{j}\left(k_{0}\right) /\left|\nabla \lambda_{j}\left(k_{0}\right)\right|$. By the definition of $\phi_{3}(k)$, it implies that $\theta$ and $\omega$ are not parallel. Therefore, we choose $\left\{e_{3}^{\prime}, e_{4}^{\prime}, \ldots, e_{d}^{\prime}\right\}$ such that $\left\{\theta, \omega, e_{3}^{\prime}, \ldots, e_{d}^{\prime}\right\}$ is a basis of $\mathbb{R}^{d}$. Changing of variables from $k$ to $\eta$ such that

$$
k=\eta_{1} \theta+\eta_{2} \omega+\tilde{\eta} \cdot \tilde{e}
$$

Denote $p_{3}(k)=\chi(k) \phi_{3}(k) \rho_{p}(k)\left\langle\mathcal{U} f(., k), \psi_{j}(., k)\right\rangle$. We obtain

$$
\begin{aligned}
K_{3 p j, \epsilon, \chi}(x+n)=\frac{1}{(2 \pi)^{d}} \int_{B^{\prime}} & \frac{p_{3}\left(\eta_{1} \theta+\eta_{2} \omega+\tilde{\eta} \cdot \tilde{e}\right)}{\lambda_{j}\left(\eta_{1} \theta+\eta_{2} \omega+\tilde{\eta} \cdot \tilde{e}\right)-(\lambda+i \epsilon)} . \\
& \psi_{j}\left(x, \eta_{1} \theta+\eta_{2} \omega+\tilde{\eta} \cdot \tilde{e}\right) e^{i\left(\eta_{1} \theta+\eta_{2} \omega+\tilde{\eta} \cdot \tilde{e}\right) \cdot n} D d \eta,
\end{aligned}
$$

where $D=\left|\operatorname{det}\left(\theta, \omega, e_{3}^{\prime}, \ldots, e_{d}^{\prime}\right)\right|$ is the Jacobian.

Again, changing of variables from $\eta$ to $\zeta$ such that $\zeta_{1}=\lambda_{j}\left(\eta_{1} \theta+\eta_{2} \omega+\tilde{\eta} \cdot \tilde{e}\right)-\lambda$ and $\left(\zeta_{2}, \tilde{\zeta}\right)=\left(\eta_{2}, \tilde{\eta}\right)$. Then,

$$
\begin{aligned}
K_{3 p j, \epsilon, \chi}(x+n)=\frac{1}{(2 \pi)^{d}} \int_{B^{\prime}} & \frac{p_{3}\left(\eta_{1}(\zeta) \theta+\zeta_{2} \omega+\tilde{\zeta} \cdot \tilde{e}\right)}{\zeta_{1}-i \epsilon} . \\
& \psi_{j}\left(x, \eta_{1}(\zeta) \theta+\zeta_{2} \omega+\tilde{\zeta} \cdot \tilde{e}\right) e^{i\left(\eta_{1}(\zeta) \theta+\zeta_{2} \omega+\tilde{\zeta} \cdot \tilde{e}\right) \cdot n} D\left|\frac{\partial \eta_{1}}{\partial \zeta_{1}}\right| d \zeta .
\end{aligned}
$$

We will see that the integral (4.35) has no stationary point with respect to variable $\zeta_{2}$. Indeed, $\partial_{\zeta_{1}} \eta_{1}=\left(\theta \cdot \nabla \lambda_{j}\right)^{-1}<\infty$ because we only consider in a small neighborhood of $k_{0}$.

Since $\zeta_{1}=\lambda_{j}\left(\eta_{1} \theta+\eta_{2} \omega+\tilde{\eta} \cdot \tilde{e}\right)-\lambda$, this implies

$$
\partial_{\zeta_{2}} \eta_{1}=\left(\omega \cdot \nabla \lambda_{j}\right) /\left(\theta \cdot \nabla \lambda_{j}\right)
$$

Thus,

$$
t(\zeta):=\partial_{\zeta_{2}}\left(\left(\eta_{1}(\zeta) \theta+\zeta_{2} \omega+\tilde{\zeta} \cdot \tilde{e}\right) \cdot n\right)=\frac{\omega \cdot \nabla \lambda_{j}}{\theta \cdot \nabla \lambda_{j}} \theta \cdot n+\omega \cdot n .
$$

Because $\omega=n /|n|$, it implies $\omega \cdot n=1$. We choose a neighborhood of $k_{0}$ small enough such that $\omega \cdot \nabla \lambda_{j}<1 / 2$ and $\theta \cdot \nabla \lambda_{j}>1 / 2$. Therefore, it implies $t(\zeta)>0$.

Applying the stationary phase method with respect to variable $\zeta_{2}$, we obtain Proposition 4.37 . 
Theorem 4.39 (Radiation condition in higher dimensions). Let $\lambda \in \mathbb{R}$ be regular and assumption in Theorem 4.33 satisfy. Then, there exists the limit $u(x)=\lim _{\epsilon \rightarrow+0} u_{\epsilon}(x)$ in $L_{\mathrm{loc}}^{2}\left(\mathbb{R}^{d}\right)$, and $u(x)$ is a solution of equation (4.3) which satisfies an asymptotic expansion

$$
\begin{aligned}
& u(x+n)=\frac{i}{(2 \pi)^{(d-1) / 2}} \sum_{j \in J(\lambda)} \sum_{k_{\omega} \in L_{j}(\omega)} \frac{A_{j}\left(x, \lambda, k_{\omega}\right)}{|n|^{(d-1) / 2}} e^{i|n| k_{\omega} \cdot \omega}+ \\
& O\left(\frac{1}{|n|^{(d+1) / 2}}\right) \quad \text { as }|n| \rightarrow \infty,
\end{aligned}
$$

uniformly in $x \in[0,1]^{d}$ and in $\omega=n /|n| \in \mathbb{S}^{d-1}$. Here,

- $J(\lambda)=\left\{j \in \mathbb{N}: \exists k \in[-\pi, \pi]^{d}, \lambda_{j}(k)=\lambda\right\}$,

- $L_{j}(\omega)=\left\{k_{\omega} \in \Gamma_{j}(\lambda): \omega=\nabla \lambda_{j}\left(k_{\omega}\right) /\left|\nabla \lambda_{j}\left(k_{\omega}\right)\right|\right\} \quad$ for $\quad j \in J(\lambda)$,

- $A_{j}\left(x, \lambda, k_{\omega}\right)=\frac{\left\langle\mathcal{U} f\left(., k_{\omega}\right), \psi_{j}\left(., k_{\omega}\right)\right\rangle e^{\frac{-i \pi}{4} \operatorname{sgn}\left(e_{l}^{\prime} \cdot \operatorname{Hess} \lambda_{j}\left(k_{\omega}\right) e_{m}^{\prime}\right)_{l, m=2}^{d}}}{\left|\nabla \lambda_{j}\left(k_{\omega}\right)\right| \sqrt{\left|K_{j}\left(k_{\omega}\right)\right|}} \psi_{j}\left(x, k_{\omega}\right)$,

where $\left(\omega, e_{2}^{\prime}, \ldots, e_{d}^{\prime}\right)$ is an orthonormal basic of $\mathbb{R}^{d}$, and $K_{j}\left(k_{\omega}\right)$ is the GaussKronecker curvature of $\Gamma_{j}(\lambda)$ at $k_{\omega}$.

Proof. From Theorem 4.33 and Propositions 4.36 and 4.37, we get the asymptotis expansion (4.37) for solution $u(x)$ of periodic Schrödinger equation (4.3).

The asymptotic expansion is uniform in $x \in[0,1]^{d}$ because of Theorem 4.24 ,

By changing slightly the above proof, we see that for $\omega \in \mathbb{S}^{d-1}$, there is a conic neighborhood $V_{\omega}$ of $\omega$ such that (4.37) holds for $n \in V_{\omega}$ with $O\left(1 /|n|^{(d+1) / 2}\right) \leq C_{\omega}|n|^{(d+1) / 2}$. Because $\mathbb{S}^{d-1}$ is compact, it implies the uniformity in $\omega \in \mathbb{S}^{d-1}$ (see also [MT06, Theorem 2.3] ).

Remark 4.40. Let us discuss the first assumption in Proposition 4.33; the existence of locally smooth band functions $\lambda_{j}(k)$ and Bloch waves $\psi_{j}(k, x)$ near the level sets $\Gamma_{j}(\lambda), j \in J(\lambda)$. By analytic perturbation theory (see Appendix A.2), these functions are separately analytic, i.e., analytic in each individual variable when others are fixed. But they are not guaranteed to be jointly analytic or even smooth. The simple example is

Example 4.41.

$$
f(x, y)= \begin{cases}\frac{x y}{x^{2}+y^{2}} & \text { if }(x, y) \neq(0,0) \\ 0 & \text { if }(x, y)=(0,0)\end{cases}
$$

is separately analytic but not continuous at $(0,0)$. 
The next example shows that even separately analytic function is smooth. It is still not jointly analytic.

Example 4.42. Consider

$$
f(x, y)= \begin{cases}x y \exp \left(-\frac{1}{x^{2}+y^{2}}\right) & \text { if }(x, y) \neq(0,0) \\ 0 & \text { if }(x, y)=(0,0)\end{cases}
$$

Then $f$ is

a) separately analytic on $\mathbb{R}^{2}$,

b) smooth on $\mathbb{R}^{2}$,

c) not jointly analytic at $(0,0)$.

This is in contradiction to complex analytic functions where separately analyticity implies jointly analyticity by Hartogs' Theorem (see [Hör90, Theorem 2.2.8]). But under a condition on uniformly bounded of holomorphic extension into complex domain, a separately real analytic function is jointly analytic by the famous Bernstein's Theorem (see [Van97, Theorem 1]). The singular set of separately real analytic function, the set of all points the function is not jointly real analytic, is characterized in [SR90, Sic90, Bło92]. It has Lebesgue measure zero and its projection into $\mathbb{R}^{d-1}$ is pluripolar. Our assumption only needs local analyticity of band functions and Bloch waves near the level set $\Gamma_{j}(\lambda), j \in J(\lambda)$ which is not so strong. The assumptions on the finite number of points in $L_{j}(\omega), j \in J(\lambda)$ and non-zero Gauss-Kronecker curvature of $\Gamma_{j}(\lambda)$ at any point $k_{\omega} \in L_{j}(\omega), j \in J(\lambda)$ are natural.

Remark 4.43. The asymptotic expansion (4.37) depends on the direction $\omega=n /|n|$. This is compatible to the result in one-dimensional case where there are only two directions $x \rightarrow \pm \infty$.

Remark 4.44. The stationary phase method can give the complete asymptotic expansion of oscillatory integral, but we only need the leading term. It is enough to prove the unique solvability.

Remark 4.45. Because we only need the leading term in asymptotic expansion, we can use Theorem A.73 to weaken the smoothness of the band functions and Bloch waves. They only need to satisfy the differentibility up to some order. 
Remark 4.46. The authors in [MT06] established asymptotics of Green functions for elliptic operators with periodic coefficients for $\lambda$ greater and close enough to the bottom of the spectrum. In this case, all assumptions in Proposition 4.33 are satisfied. The asymptotic expansion (4.37) becomes simple because the set $J(\lambda)=\{1\}$ and $L_{1}(\omega)$ contains only one point for all $\omega \in \mathbb{S}^{d-1}$ (see also [Gho02]).

Theorem 4.47 ([MT06, Theorem 1.1]). Assume $\lambda$ is greater and close enough to the bottom of the spectrum of elliptic operator with periodic coefficients $H$. Then, the limit $(H-(\lambda+i 0))^{-1} f(x):=\lim _{\epsilon \rightarrow+0}(H-\lambda-i \epsilon)^{-1} f(x)$ in $L_{\text {loc }}^{2}\left(\mathbb{R}^{d}\right)$ exists for $f \in L^{2}\left(\mathbb{R}^{d}\right)$ with compact support, and the integral kernel $G_{\lambda+i 0}(x, y)$ of $(H-(\lambda+i 0))^{-1}$ admits the following asymptotics as $|x-y| \rightarrow \infty$

$$
G_{\lambda+i 0}(x, y)=\frac{e^{i \pi(3-d) / 4}}{(2 \pi)^{(d-1) / 2}} \frac{\psi_{1}\left(x, k_{\omega}\right) \overline{\psi_{1}\left(y, k_{\omega}\right)} e^{i(x-y) \cdot k_{\omega}}}{|x-y|^{(d-1) / 2}\left|\nabla \lambda_{j}\left(k_{\omega}\right)\right| \sqrt{\left|K_{j}\left(k_{\omega}\right)\right|}}+O\left(\frac{1}{|x-y|^{(d+1) / 2}}\right),
$$

where $k_{\omega} \in \Gamma_{1}(\lambda)$ is a unique point satisfying $\omega=\nabla \lambda_{1}\left(k_{\omega}\right) /\left|\nabla \lambda_{1}\left(k_{\omega}\right)\right|$.

In our case, we get a similar asymptotic expansion for the outgoing solution of the periodic Schrödinger equation.

Proposition 4.48. Assume $\lambda$ is greater and close enough to the bottom of the spectrum. Then, the solution $u(x)=\lim _{\epsilon \rightarrow+0} u_{\epsilon}(x)$ of (4.3) has an asymptotic expansion as $|n| \rightarrow \infty$

$$
\begin{gathered}
u(x+n)=\frac{e^{i \pi(3-d) / 4}}{(2 \pi)^{(d-1) / 2}} \frac{\left\langle\mathcal{U} f\left(., k_{\omega}\right), \psi_{1}\left(., k_{\omega}\right)\right\rangle}{|n|^{(d-1) / 2}\left|\nabla \lambda_{j}\left(k_{\omega}\right)\right| \sqrt{\left|K_{j}\left(k_{\omega}\right)\right|}} \psi_{1}\left(x, k_{\omega}\right) e^{i n \mid k_{\omega} \cdot \omega}+ \\
O\left(\frac{1}{|n|^{(d+1) / 2}}\right),
\end{gathered}
$$

where $k_{\omega} \in \Gamma_{1}(\lambda)$ is a unique point satisfying $\omega=\nabla \lambda_{1}\left(k_{\omega}\right) /\left|\nabla \lambda_{1}\left(k_{\omega}\right)\right|$.

Remark 4.49. In [KR12, KKR17], the authors considered the asymptotics of Green function at spectral edge and spectral gap interior. They made assumptions on spectral edge which is known as the conjecture for generic Schödinger operator (see also Kuc16, Conjecture 5.25]).

Conjecture 1. Generically, for a self-adjoint second-order elliptic operator with real periodic coefficients on $\mathbb{R}^{d}$, the spectral gap's endpoints

i) are attained by a singer band,

ii) are isolated 
iii) are non-degenerated, i.e., have non-degenerate Hessian.

The condition i) holds for generic Schödinger operator [KR00]. The condition ii) and iii) are usually assumed in both mathematics and physics but it is still unproved (for more discussion see [Kuc16]).

The main idea to derive the asymptotic expansion of Green function in [KR12, KKR17] is to use the analyticity of the band functions and Bloch waves. In our case, under the assumptions in Conjecture 1, it is easy to derive the asymptotic expansion of the solution when the spectral parameter is in the spectrum and close enough to the spectral edge. The formula is similar to (4.38).

The next result is the unique solvability for solution of (4.3) under the radiation condition in Theorem 4.39 .

Theorem 4.50 (Uniqueness for outgoing solution). Let $\lambda \in \mathbb{R}$ be regular and the assumption in Theorem 4.33 satisfy. Then, there is a unique solution to $-\Delta u+V(x) u-\lambda u=$ $f(x)$ satisfying the asymptotic expansion

$$
\begin{aligned}
& u(x+n)=\frac{i}{(2 \pi)^{(d-1) / 2}} \sum_{j \in J(\lambda)} \sum_{k_{\omega} \in L_{j}(\omega)} \frac{A_{j}\left(x, \lambda, k_{\omega}\right)}{|n|^{(d-1) / 2}} e^{i n \mid k_{\omega} \cdot \omega}+ \\
& O\left(\frac{1}{|n|^{(d+1) / 2}}\right) \quad \text { as }|n| \rightarrow \infty,
\end{aligned}
$$

uniformly in $x \in[0,1]^{d}$ and in $\omega=n /|n| \in \mathbb{S}^{d-1}$.

Here,

- $J(\lambda)=\left\{j \in \mathbb{N}: \exists k \in[-\pi, \pi]^{d}, \lambda_{j}(k)=\lambda\right\}$,

- $L_{j}(\omega)=\left\{k_{\omega} \in \Gamma_{j}(\lambda): \omega=\nabla \lambda_{j}\left(k_{\omega}\right) /\left|\nabla \lambda_{j}\left(k_{\omega}\right)\right|\right\} \quad$ for $\quad j \in J(\lambda)$,

- $A_{j}\left(x, \lambda, k_{\omega}\right)=\frac{\left\langle\mathcal{U} f\left(., k_{\omega}\right), \psi_{j}\left(., k_{\omega}\right)\right\rangle e^{\frac{-i \pi}{4} \operatorname{sgn}\left(e_{l}^{\prime} \cdot \operatorname{Hess} \lambda_{j}\left(k_{\omega}\right) e_{m}^{\prime}\right)_{l, m=2}^{d}}}{\left|\nabla \lambda_{j}\left(k_{\omega}\right)\right| \sqrt{\left|K_{j}\left(k_{\omega}\right)\right|}} \psi_{j}\left(x, k_{\omega}\right)$,

where $\left(\omega, e_{2}^{\prime}, \ldots, e_{d}^{\prime}\right)$ is an orthonormal basic of $\mathbb{R}^{d}$, and $K_{j}\left(k_{\omega}\right)$ is the GaussKronecker curvature of $\Gamma_{j}(\lambda)$ at $k_{\omega}$.

Proof. Suppose there are two solutions $u(x), v(x)$ satisfying the asymptotic expansion (4.39). Then, $w(x)=u(x)-v(x)$ satisfies the homogeneous equation $-\Delta u+V(x) u-\lambda u=$ 0 .

Using (4.39), it implies that $w$ is in $L^{2}\left(\mathbb{R}^{d}\right)$. Therefore, the spectral theorem implies $w(x) \equiv 0$. That means $u(x) \equiv v(x)$.

Thus, we get the unique solution satisfying the asymptotic expansion (4.39). 
Remark 4.51. The asymptotic expansion (4.39) is called the outgoing radiation condition. If we take the limit $u_{\epsilon}$ as $\epsilon \rightarrow-0$ instead of $\epsilon \rightarrow+0$, then we will get incoming solution. The incoming radiation condition can be derived by using the same argument as before. The stationary points for the incoming solution satisfy a condition $\omega=-\nabla \lambda_{j}\left(k_{\omega}\right) /\left|\nabla \lambda_{j}\left(k_{\omega}\right)\right|$.

Theorem 4.52 (Incoming radiation condition). Let $\lambda \in \mathbb{R}$ be regular. Assume that there exists a system of locally smooth band functions $\lambda_{j}(k)$ and Bloch waves $\psi_{j}(k, x)$ near the level sets $\Gamma_{j}(\lambda), j \in J(\lambda)$; the number of points in $L_{j}(-\omega)$ is finite for all $j \in J(\lambda), \omega \in$ $\mathbb{S}^{d-1}$, and Gauss-Kronecker curvature of $\Gamma_{j}(\lambda)$ oriented by $-\nabla \lambda_{j}(k) /\left|\nabla \lambda_{j}(k)\right|$ at any point $k_{\omega} \in L_{j}(-\omega), j \in J(\lambda)$ is non-zero. Then, the limit $u(x)=\lim _{\epsilon \rightarrow-0} u_{\epsilon}(x)$ exists in $L_{\mathrm{loc}}^{2}\left(\mathbb{R}^{d}\right)$ and it is a solution to (4.3) which satisfies an asymptotic expansion

$$
\begin{aligned}
& u(x+n)=\frac{-i}{(2 \pi)^{(d-1) / 2}} \sum_{j \in J(\lambda)} \sum_{k_{\omega} \in L_{j}(-\omega)} \frac{A_{j}\left(x, \lambda, k_{\omega}\right)}{|n|^{(d-1) / 2}} e^{i|n| k_{\omega} \cdot \omega}+ \\
& O\left(\frac{1}{|n|^{(d+1) / 2}}\right) \quad \text { as }|n| \rightarrow \infty,
\end{aligned}
$$

uniformly in $x \in[0,1]^{d}$ and in $\omega=n /|n| \in \mathbb{S}^{d-1}$.

Here,

- $J(\lambda)=\left\{j \in \mathbb{N}: \exists k \in[-\pi, \pi]^{d}, \lambda_{j}(k)=\lambda\right\}$,

- $L_{j}(-\omega)=\left\{k_{\omega} \in \Gamma_{j}(\lambda): \omega=-\nabla \lambda_{j}\left(k_{\omega}\right) /\left|\nabla \lambda_{j}\left(k_{\omega}\right)\right|\right\} \quad$ for $\quad j \in J(\lambda)$.

- $A_{j}\left(x, \lambda, k_{\omega}\right)=\frac{\left\langle\mathcal{U} f\left(., k_{\omega}\right), \psi_{j}\left(., k_{\omega}\right)\right\rangle e^{\frac{i \pi}{4} \operatorname{sgn}\left(e_{l}^{\prime} \cdot \operatorname{Hess} \lambda_{j}\left(k_{\omega}\right) e_{m}^{\prime}\right)_{l, m=2}^{d}}}{\left|\nabla \lambda_{j}\left(k_{\omega}\right)\right| \sqrt{\left|K_{j}\left(k_{\omega}\right)\right|}} \psi_{j}\left(x, k_{\omega}\right)$,

where $\left(\omega, e_{2}^{\prime}, \ldots, e_{d}^{\prime}\right)$ is an orthonormal basic of $\mathbb{R}^{d}$, and $K_{j}\left(k_{\omega}\right)$ is the GaussKronecker curvature of $\Gamma_{j}(\lambda)$ at $k_{\omega}$.

We also get the unique solvability under the incoming radiation condition.

Theorem 4.53 (Uniqueness for incoming solution). Let $\lambda \in \mathbb{R}$ be regular and the assumption in Theorem 4.52 satisfy. Then, there is a unique solution to $-\Delta u+V(x) u-\lambda u=$ $f(x)$ satisfying the asymptotic expansion

$$
\begin{aligned}
& u(x+n)=\frac{-i}{(2 \pi)^{(d-1) / 2}} \sum_{j \in J(\lambda)} \sum_{k_{\omega} \in L_{j}(-\omega)} \frac{A_{j}\left(x, \lambda, k_{\omega}\right)}{|n|^{(d-1) / 2}} e^{i|n| k_{\omega} \cdot \omega}+ \\
& O\left(\frac{1}{|n|^{(d+1) / 2}}\right) \quad \text { as }|n| \rightarrow \infty,
\end{aligned}
$$

uniformly in $x \in[0,1]^{d}$ and in $\omega=n /|n| \in \mathbb{S}^{d-1}$. 
Remark 4.54. From (4.37), the structure of the outgoing solution is

$$
\begin{aligned}
& u(x+n)=\frac{1}{|n|^{(d-1) / 2}} \sum_{j \in J(\lambda)} \sum_{k_{\omega} \in L_{j}(\omega)} c_{j, k_{\omega}} \psi_{j}\left(x, k_{\omega}\right) e^{i|n| k_{\omega} \cdot \omega}+ \\
&+O\left(\frac{1}{|n|^{(d+1) / 2}}\right) \quad \text { as }|n| \rightarrow \infty,
\end{aligned}
$$

uniformly in $x \in[0,1]^{d}$ and in $\omega=n /|n| \in \mathbb{S}^{d-1}$.

Here,

- $J(\lambda)=\left\{j \in \mathbb{N}: \exists k \in[-\pi, \pi]^{d}, \lambda_{j}(k)=\lambda\right\}$,

- $L_{j}(\omega)=\left\{k_{\omega} \in \Gamma_{j}(\lambda): \omega=\nabla \lambda_{j}\left(k_{\omega}\right) /\left|\nabla \lambda_{j}\left(k_{\omega}\right)\right|\right\} \quad$ for $\quad j \in J(\lambda)$.

The sum above is a finite linear combination of Bloch waves with positive group velocity. I conjecture that the condition (2) is a strong radiation condition for outgoing solution. It is hard to prove the unique solvability of the periodic Schrödinger equation $-\Delta u+V u-\lambda u=f(x)$ under (2).

Conjecture 2. Let $\lambda \in \mathbb{R}$ be regular and the assumptions in Theorem 4.33 satisfy. Then, there is a unique solution to $-\Delta u+V(x) u-\lambda u=f(x)$ satisfying the asymptotic expansion

$$
u(x+n)=\frac{1}{|n|^{(d-1) / 2}} \sum_{j \in J(\lambda)} \sum_{k_{\omega} \in L_{j}(\omega)} c_{j, k_{\omega}} \psi_{j}\left(x, k_{\omega}\right) e^{i|n| k_{\omega} \cdot \omega}+O\left(\frac{1}{|n|^{(d+1) / 2}}\right) \quad \text { as }|n| \rightarrow \infty,
$$

uniformly in $x \in[0,1]^{d}$ and in $\omega=n /|n| \in \mathbb{S}^{d-1}$. 


\section{A Appendix}

In the Appendix, we recall some results on spectral theory of self-adjoint operators which are useful to the study of periodic Schrödinger operators; analytic perturbation theory for analyzing the band functions and Bloch waves; and the stationary phase method which we use to derive the asymptotic expansion for solutions of the periodic Schrödinger equation.

\section{A.1 Spectral theory of self-adjoint operators}

Spectral theory of self-adjoint operators is a clasical topic and can be found in many textbooks. Here we follow [RS80, HS96].

\section{A.1.1 Self-adjoint operators}

Let $\mathscr{H}$ be a Hilbert space with an inner product $\langle.,$.$\rangle and H: D(H) \rightarrow \mathscr{H}$ a densely defined linear operator, i.e., the closure $\overline{D(H)}=\mathscr{H}$.

Definition A.1. The graph of a linear transformation $H$ is the set

$$
\Gamma(H)=\{(\phi, H \phi): \phi \in D(H)\} .
$$

The graph of $H$ is a subset of $\mathscr{H} \times \mathscr{H}$ which is a Hilbert space with an inner product

$$
\left\langle\left(\phi_{1}, \psi_{1}\right),\left(\phi_{2}, \psi_{2}\right)\right\rangle_{\mathscr{H} \times \mathscr{H}}=\left\langle\phi_{1}, \phi_{2}\right\rangle+\left\langle\psi_{1}, \psi_{2}\right\rangle
$$

The operator $H$ is said to be closed if $\Gamma(H)$ is the closed subset of $\mathscr{H} \times \mathscr{H}$.

Definition A.2. Let $H_{1}, H$ be operators on $\mathscr{H}$. If $\Gamma\left(H_{1}\right) \supseteq \Gamma(H)$, then $H_{1}$ is said to be an extension of $H$ and we write $H_{1} \supseteq H$. Equivalently, $H_{1} \supseteq H$ if and only if $D\left(H_{1}\right) \supseteq D(H)$ and $H_{1} \phi=H \phi$ for all $\phi \in D(H)$. 
Definition A.3. An operator $H$ is closable if it has a closed extension. Every closable operator has a smallest closed extension, called its closure, which we denote by $\bar{H}$.

Definition A.4. The adjoint of $H, H^{*}$, is defined on the domain

$$
D\left(H^{*}\right)=\{\phi \in \mathscr{H}: \exists C>0,|\langle H \psi, \phi\rangle| \leq C\|\psi\| \quad \text { for all } \psi \in D(H)\} .
$$

By the Riesz representation theorem, for each $\phi \in D\left(H^{*}\right)$ there is a unique $\eta \in \mathscr{H}$ such that

$$
(H \psi, \phi)=(\psi, \eta) \quad \text { for all } \psi \in D(H) .
$$

The adjoint $H^{*}: D\left(H^{*}\right) \rightarrow \mathscr{H}$ is given by

$$
H^{*} \phi=\eta \text {. }
$$

Remark A.5. If the domain $D(H)$ is not dense, then the adjoint is not uniquely defined because the Riesz representation theorem is not applicable.

Proposition A.6. Let $H$ be a densely defined operator on a Hilbert space $\mathscr{H}$. Then,

i) $H^{*}$ is closed,

ii) $H$ is closable if and only if $D\left(H^{*}\right)$ is dense in which $\bar{H}=H^{* *}$,

iii) if $H$ is closable, then $(\bar{H})^{*}=H^{*}$.

Definition A.7. A densely defined operator $H$ on a Hilbert space $\mathscr{H}$ is called symmetric (or Hermitian) if $H \subseteq H^{*}$, i.e., $D(H) \subseteq D\left(H^{*}\right)$ and $H \phi=H^{*} \phi$ for all $\phi \in D(H)$. Equivalently, $H$ is symmetric if and only if

$$
\langle H \phi, \psi\rangle=\langle\phi, H \psi\rangle \quad \text { for all } \phi, \psi \in D(H) .
$$

Definition A.8. $\quad$ i) $H$ is called self-adjoint if $H=H^{*}$, i.e., $H$ is symmetric and $D(H)=D\left(H^{*}\right)$.

ii) A symmetric operator $H$ is called essentially self-adjoint if its closure $\bar{H}$ is selfadjoint. If $H$ is closed, a subset $D \subset D(H)$ is called a core of $H$ if $\overline{H_{\uparrow D}}=H$.

Remark A.9. i) A symmetric operator is always closable and

$$
H \subseteq H^{* *} \subseteq H^{*}
$$


ii) For closed symmetric operator

$$
H=H^{* *} \subseteq H^{*} .
$$

iii) For self-adjoint operator

$$
H=H^{* *}=H^{*} .
$$

Example A.10. Let $\mathscr{H}=L^{2}([0,1])$ and let $H_{1}$ be the operator $H_{1}=-d / d x^{2}$ with a domain

$$
\begin{gathered}
D\left(H_{1}\right)=\left\{f \in \mathscr{H}: f, f^{\prime} \text { is absolutely continuous in }[0,1], f^{\prime \prime} \in L^{2}([0,1])\right. \text { and } \\
\left.\qquad u^{(k)}(0)=u^{(k)}(1)=0 \text { for } k=0,1\right\} .
\end{gathered}
$$

It is easy to see that this operator is symmetric but not self-adjoint because

$$
D\left(H_{1}^{*}\right)=\left\{g \in \mathscr{H}: g, g^{\prime} \text { is absolutely continuous, } g^{\prime \prime} \in L^{2}([0,1])\right\}
$$

Example A.11. i) Let $V \in L_{\text {loc }}^{2}\left(\mathbb{R}^{d}\right)$ and be bounded from below. Then, the Schrödinger operator $H=-\Delta+V$ is essentially self-adjoint with a domain $C_{\mathrm{c}}^{\infty}\left(\mathbb{R}^{d}\right)$.

ii) The Laplacian $-\Delta$ on $L^{2}\left(\mathbb{R}^{d}\right)$ is self-adjoint with the domain $H^{2}\left(\mathbb{R}^{d}\right)$.

Theorem A.12 ([RS80], the basic criterion for self-adjointness). Let $H$ be a symmetric operator on a Hilbert space $\mathscr{H}$. Then, the following three statements are equivalent

i) $H$ is self-adjoint,

ii) $H$ is closed and $\operatorname{ker}\left(H^{*} \pm i\right)=\{0\}$,

iii) $\operatorname{ran}(H \pm i)=\mathscr{H}$.

Definition A.13 ([RS75]). Let $H, H_{0}$ be densely defined operators on a Hilbert space $\mathscr{H} . H_{0}$ is said to be $H$-bounded if $D\left(H_{0}\right) \supseteq D(H)$ and there exist $a \geq 0$ and $b \in \mathbb{R}$ such that

$$
\left\|H_{0} \phi\right\| \leq a\|H \phi\|+b\|\phi\| \quad \text { for all } \phi \in D(H)
$$

The infimum of such $a$ in above inequality is called the relative $H$-bound of $H_{0}$.

Theorem A.14 (Kato-Rellich theorem). Let $H$ be self-adjoint, and let $H_{0}$ be symmetric, and $H$-bounded with relative bound less than 1 . Then, $H+H_{0}$ is self-adjoint on $D(H)$. 
Applying Kato-Rellich theorem for the Schrödinger operator with relatively bounded potentials, the following theorems hold

Theorem A.15 ([HS96, Theorem 13.7]). A real potential $V=L^{2}\left(\mathbb{R}^{3}\right)+L^{\infty}\left(\mathbb{R}^{3}\right)$ is $(-\Delta)$ bounded with relative bound zero. Consequently, the Schrödinger operator $H=-\Delta+V$ defined on $D(-\Delta)=H^{2}\left(\mathbb{R}^{3}\right)$ is self-adjoint.

Theorem A.16 ([RS78, Theorem XIII.96]). Let $p=2$ if $d \leq 3, p>2$ if $d=4$ and $p>d / 2$ if $n \geq 5$. Then, any real-valued function $V$ on $\mathbb{R}^{d}$ that is uniformly locally $L^{p}$ is a $(-\Delta)$-bounded operator with relative bound zero. A consequence is that operator $-\Delta+V(x)$ on $L^{2}\left(\mathbb{R}^{d}\right)$ is self-adjoint with the domain $H^{2}\left(\mathbb{R}^{d}\right)$.

\section{A.1.2 The spectral theorem of self-adjoint operators}

First, we recall the definition of the spectrum of a linear operator $H$ on a Banach space $\mathscr{H}$.

Definition A.17. Let $H$ be a linear operator on $\mathscr{H}$ with the domain $D(H)$.

1) $H$ is invertible if there is a bounded operator, called $H^{-1}$, such that $H^{-1}: \mathscr{H} \rightarrow$ $D(H), H H^{-1}=I_{X}$, and $H^{-1} H=I_{D(H)}$.

2) The spectrum of $H, \sigma(H)$, is the set of all points $\lambda \in \mathbb{C}$ for which $H-\lambda$ is not invertible.

3) The resolvent set of $H, \rho(H)$, is the set of all points $\lambda \in \mathbb{C}$ for which $H-\lambda$ is invertible. If $\lambda \in \rho(H)$, then the inverse of $H-\lambda$ is called the resolvent of $H$ at $\lambda$ and written as $R_{H}(\lambda)=(H-\lambda)^{-1}$.

Proposition A.18. The resolvent set $\rho(H)$ is an open subset of $\mathbb{C}$ (and hence $\sigma(H)$ is closed), and $R_{H}(\lambda)$ is an analytic operator-valued function of $\lambda$ on $\rho(H)$. Moreover,

$$
\sigma(H) \cup \rho(H)=\mathbb{C}, \quad \sigma(H) \cap \rho(H)=\varnothing .
$$

Definition A.19. 1) If $\lambda \in \sigma(H)$ satisfies $\operatorname{ker}(H-\lambda) \neq\{0\}$, then $\lambda$ is an eigenvalue of $H$ and any $\phi \in \operatorname{ker}(H-\lambda)$ is called an eigenvector of $H$ for $\lambda$. Moreover, $\operatorname{dim}(\operatorname{ker}(H-\lambda))$ is called the geometric multiplicity of $\lambda$ and $\operatorname{ker}(H-\lambda)$ is the geometric eigenspace of $H$ at $\lambda$. 
2) The discrete spectrum of $H, \sigma_{\text {disc }}(H)$, is the set of all eigenvalues of $H$ with finite algebraic multiplicity and which are isolated points of $\sigma(H)$.

3) The essential spectrum is defined as the complement of $\sigma_{\text {ess }}(H)$ in $\sigma(H)$, i.e., $\sigma_{\mathrm{ess}}=\sigma(H) \backslash \sigma_{\mathrm{disc}}(H)$.

Remark A.20. The algebraic multiplicity of an isolated point $\lambda \in \sigma(H)$ is the dimension of Riesz projection $P_{\lambda}=(2 \pi i)^{-1} \oint_{\Gamma} R_{H}(z) d z$, where $\Gamma$ is a simple closed contour around $\lambda$ such that the closure of the region bounded by $\Gamma$ contains only $\lambda$ in the spectrum.

The geometric multiplicity of an eigenvalue is always less than or equal to its algebraic multiplicity. For self-adjoint operators, they are equal.

If the operator $H$ on the Hilbert space $\mathscr{H}$ is self-adjoint, then the spectrum can be decomposed by using the projection-valued measure associated with $H$. Let $\mathscr{B}(\mathbb{R})$ be the Borel $\sigma$-algebra of $\mathbb{R}$.

Definition A.21 ([RS80]). A projection-valued measure is the family of operators $\{E(\Omega), \Omega \in$ $\mathscr{B}(\mathbb{R})\}$ on the Hilbert space $\mathscr{H}$ such that

i) each $E(\Omega)$ is an orthogonal projection,

ii) $E(\varnothing)=0, E((-\infty, \infty))=I$,

iii) if $\Omega=\bigcup_{k=1}^{\infty} \Omega_{k}$ with $\Omega_{k} \cap \Omega_{l}=\varnothing$ for all $k \neq l$, then

$$
E(\Omega)=\underset{N \rightarrow \infty}{\mathrm{s}-\lim _{k=1}} \sum_{k}^{N} E\left(\Omega_{k}\right),
$$

iv) $E\left(\Omega_{1}\right) E\left(\Omega_{2}\right)=E\left(\Omega_{1} \cap \Omega_{2}\right)$.

Denote $E((-\infty, \lambda])$ by $E_{\lambda}$.

Proposition A.22 ([RS80]). Let $\{E(\Omega), \Omega \in \mathscr{B}(\mathbb{R})\}$ be a projection-valued measure.

Define a linear operator $H: D(H) \subseteq \mathscr{H} \rightarrow \mathscr{H}$ by

$$
D(H)=\left\{\phi \in \mathscr{H}: \int_{-\infty}^{\infty} \lambda^{2} d\left\langle E_{\lambda} \phi, \phi\right\rangle<\infty\right\},
$$

and for $\phi \in D(H)$,

$$
\langle H \phi, \psi\rangle=\int_{-\infty}^{\infty} \lambda d\left\langle E_{\lambda} \phi, \psi\right\rangle \quad \forall \psi \in \mathscr{H}
$$


Then, $H$ is self-adjoint.

$\{E(\Omega), \Omega \in \mathscr{B}(\mathbb{R})\}$ is called the projection-valued measure associated with the selfadjoint operator $H$. One writes

$$
H=\int_{-\infty}^{\infty} \lambda d E_{\lambda}
$$

Theorem A.23 ([RS80], functional calculus). Let $H$ be a self-adjoint operator on a Hilbert space $\mathscr{H},\{E(\Omega), \Omega \in \mathscr{B}(\mathbb{R})\}$ be the projection-valued measure associated with $H$. Define $\Phi(f)=\int_{\sigma(H)} f(\lambda) d E_{\lambda}$ for a measurable function $f: \sigma(H) \rightarrow \mathbb{C}$ with its natural domain. Then,

- $\Phi(f)$ is a normal operator.

- $\Phi(f)$ is self-adjoint if $f$ is real-valued.

- $\Phi(f)$ is unitary if $|f|=1$.

- $\Phi(f)$ is bounded if $f$ is bounded and then $\|\Phi(f)\|_{L(\mathscr{H})}=\|f\|_{L^{\infty}(\sigma(H))}$

- $\Phi(1)=I, \Phi(\lambda)=H$.

- $\Phi(f)+\Phi(g)=\Phi(f+g) \quad$ on $D(\Phi(f)) \bigcap D(\Phi(g))$.

- $\Phi(f)^{*}=\Phi(\bar{f})$.

- $\Phi(f) \Phi(g)=\Phi(f g) \quad$ on $D(\Phi(f g))$.

We denote $\Phi(f)$ by $f(H)$.

Theorem A.24 ([RS80], spectral theorem - Projection-valued measure form). There is one to one correspondence between the self-adjoint operator $H$ and the projectionvalued measure $\{E(\Omega), \Omega \in \mathscr{B}(\mathbb{R})\}$ on $\mathscr{H}$ given by the map

$$
E \mapsto H=\int_{-\infty}^{\infty} \lambda d E_{\lambda}
$$

Remark A.25. $d E_{\lambda}$ is called the spectral measure of $H$.

The spectral measure can be used to get the spectral decomposition and classify the spectrum of the self-adjoint operator $H$.

Proposition A.26 ([RS80]). Let $H$ be a self-adjoint operator on $\mathscr{H}, d \mu_{\phi}=d\left\langle E_{\lambda} \phi, \phi\right\rangle$. Then,

$$
\mathscr{H}=\mathscr{H}_{\mathrm{pp}}(H) \oplus \mathscr{H}_{\mathrm{ac}}(H) \oplus \mathscr{H}_{\mathrm{sc}}(H)
$$

where 
- $\mathscr{H}_{\mathrm{pp}}(H)=\left\{\phi \in \mathscr{H}: d \mu_{\phi}\right.$ is pure point $\}$,

- $\mathscr{H}_{\mathrm{ac}}(H)=\left\{\phi \in \mathscr{H}: d \mu_{\phi}\right.$ is absolutely continuous $\}$,

- $\mathscr{H}_{\mathrm{sc}}(H)=\left\{\phi \in \mathscr{H}: d \mu_{\phi}\right.$ is singularly continuous $\}$.

Each of these subspaces reduces $H$, i.e., $\left.H\right|_{\mathscr{H}_{\mathrm{pp}}}$ has a complete set of eigenvectors, $\left.H\right|_{\mathscr{H}_{\mathrm{ac}}}$ has only absolutely continuous spectral measure and $\left.H\right|_{\mathscr{H} \text { sc }}$ has only singularly continuous spectral measure.

Remark A.27. In physics, it holds $\mathscr{H}_{\mathrm{sc}}(H)=\{0\}$ as a rule. Actually, it is fulfilled for large class of Schrödinger operators (for more details see [RS78]).

Theorem A.28 ([RS80, Stone's formula]). Let $H$ be a bounded or unbounded selfadjoint operator. Then,

$$
\operatorname{sim}_{\epsilon \rightarrow+0} \int_{a}^{b}\left[(H-\lambda-i \epsilon)^{-1}-(H-\lambda+i \epsilon)^{-1}\right] d \lambda=\frac{1}{2}\left[E_{[a, b]}+E_{(a, b)}\right] .
$$

Definition A.29.

$$
\begin{aligned}
\sigma_{\mathrm{pp}}(H) & =\sigma\left(\left.H\right|_{\mathscr{H}_{\mathrm{pp}}(H)}\right) \\
\sigma_{\mathrm{cont}}(H) & =\sigma\left(\left.H\right|_{\mathscr{H}_{\mathrm{cont}}(H) \equiv \mathscr{H}_{\mathrm{sc}}(H) \oplus \mathscr{H}_{\mathrm{as}}(H)}\right) \\
\sigma_{\mathrm{ac}}(H) & =\sigma\left(\left.H\right|_{\mathscr{H}_{\mathrm{ac}}(H)}\right) \\
\sigma_{\mathrm{sc}}(H) & =\sigma\left(\left.H\right|_{\mathscr{H}_{\mathrm{sc}}(H)}\right)
\end{aligned}
$$

Remark A.30. $\sigma_{\mathrm{pp}}$ consists of all eigenvalues of the operator $H$ and limit points of these eigenvalues.

Proposition A.31 ([RS80, HS96]). Let $H$ be self-adjoint on a Hilbert space $\mathscr{H}$. Then $\sigma(H) \subseteq \mathbb{R}$ and eigenfunctions corresponding to distinct eigenvalues are orthogonal.

Proposition A.32 ([RS80], spectral decomposition).

$$
\begin{aligned}
\sigma_{\text {cont }}(H) & =\sigma_{\mathrm{ac}}(H) \cup \sigma_{\mathrm{sc}}(H) . \\
\sigma(H) & =\sigma_{\mathrm{pp}}(H) \cup \sigma_{\text {cont }}(H) .
\end{aligned}
$$

Remark A.33. The spectral decomposition above needs not be disjoint. An eigenvalue $\lambda$ which is in $\sigma_{\mathrm{ac}}(H)$ is called an embedded eigenvalue into the absolutely continuous spectrum. 
Definition A.34. An operator $H$ on a Hilbert space is positive, $H \geq 0$, if $\langle u, H u\rangle \geq 0$ for all $u \in D(H)$.

Theorem A.35 ([HS96, Proposition 5.12]). Let $H$ be a self-adjoint operator. Then, $A \geq 0$ if and only if $\sigma(H) \subseteq[0, \infty)$.

Example A.36. The Laplace operator $-\Delta$ on $L^{2}\left(\mathbb{R}^{d}\right)$ is self-adjoint with the domain $H^{2}\left(\mathbb{R}^{d}\right)$. The spectrum $\sigma(-\Delta)=[0, \infty)$ is purely absolutely continuous.

Using the projection-valued measure $\{E(\Omega), \Omega \in \mathscr{B}(\mathbb{R})\}$, the spectrum of self-adjoint operator $H$ can be realized as follow:

Proposition A.37 ([RS80]). Let $\lambda$ be in the spectrum of a self-adjoint operator $H$ on a Hilbert space $\mathscr{H}$. Then,

1) $\lambda \in \sigma_{\mathrm{ess}}(H)$ if $E((\lambda-\epsilon, \lambda+\epsilon))$ is infinite dimensional for all $\epsilon>0$,

2) $\lambda \in \sigma_{\text {disc }}(H)$ if $E((\lambda-\epsilon, \lambda+\epsilon))$ is finite dimensional for some $\epsilon>0$.

Theorem A.38 ([RS80, Theorem VII.10, VII.11]). i) $\lambda \in \sigma_{\text {disc }}(H)$ if and only if both the following statements hold

a) $\lambda$ is an isolated point of $\sigma(H)$, i.e., for some $\epsilon>0, \sigma(H) \cap(\lambda-\epsilon, \lambda+\epsilon)=\{\lambda\}$,

b) $\lambda$ is an eigenvalue of finite multiplicity, i.e., $\{\phi: H \phi=\lambda \phi\}$ is finite dimensional.

ii) $\lambda \in \sigma_{\mathrm{ess}}(H)$ if and only if one of the following statements holds

a) $\lambda \in \sigma_{\text {cont }}(H)$,

b) $\lambda$ is a limit point of eigenvalues of $H$,

c) $\lambda$ is an eigenvalue of infinite multiplicity.

Example A.39 ([HS96]). Assume that $V$ is real and $\Delta$-bounded with relative $\Delta$-bound less than 1 , and $V(x) \rightarrow 0$ as $|x| \rightarrow \infty$. Then, $H=-\Delta+V$ is self-adjoint on $H^{2}\left(\mathbb{R}^{d}\right)$ and

$$
\sigma_{\mathrm{ess}}(H)=\sigma(-\Delta)=[0, \infty) .
$$

Theorem A.40 ([RS80]). Let H be a self-adjoint operator on a Hilbert space $\mathscr{H}$. Then,

i) $\lambda \in \sigma(H)$ if and only if there exists a sequence $\left\{\psi_{j}\right\} \subset D(A),\left\|\psi_{j}\right\|=1$, such that $\lim _{n \rightarrow \infty}\left\|(A-\lambda) \psi_{j}\right\|=0$. 
ii) $\lambda$ is an eigenvalue of $A$ with finite multiplicity if and only if there exists only a finite number of linear independent functions $\psi_{j} \in D(H)$ such that $(A-\lambda) \psi_{j}=0$.

iii) $\lambda$ is an eigenvalue with infinite multiplicity if and only if there exists a sequence of linearly independent functions $\left\{\psi_{j}\right\}_{j \in \mathbb{N}} \subset D(H)$ such that $(A-\lambda) \psi_{j}=0$.

Theorem A.41 (Weyl's criterion). Let H be a self-adjoint operator on a Hilbert space $\mathscr{H}$. Then, $\lambda \in \sigma_{\mathrm{ess}}(H)$ if and only if there exists a sequence $\left\{\psi_{j}\right\} \subset D(H)$ such that $\left\|\psi_{j}\right\|=1$, the weak limit $\psi_{j} \stackrel{w}{\rightarrow} 0$ and strong limit $(H-\lambda) \psi_{j} \stackrel{s}{\rightarrow} 0$ hold.

Next, we recall some properties and spectral theorem of compact operators.

Definition A.42 ([RS80]). A linear operator $H$ on a Hilbert space $\mathscr{H}$ is called compact if $H$ maps a bounded set into a pre-compact set in $\mathscr{H}$. Equivalently, $H$ is compact if and only if for every bounded sequence $\left\{x_{n}\right\} \subset \mathscr{H},\left\{H x_{n}\right\}$ has a subsequence convergent in $\mathscr{H}$.

Theorem A.43 ([HS96], spectral theorem of compact operator). Let $H$ be compact and self-adjoint. Then, the spectrum of $H$ consists of non-zero isolated eigenvalues of finite multiplicity with the only accumulation point at zero. Moreover,

i) $\|H\|=\sup \{|\lambda|: \lambda \in \sigma(H)\}$.

ii) Let $\left\{\lambda_{j}\right\}$ be the sequence of eigenvalues of $H$ and

$$
P_{j}: \mathscr{H} \rightarrow \operatorname{ker}\left(H-\lambda_{j} I\right)
$$

the orthogonal projections onto the corresponding eigenspaces. Then, $P_{j}$ is finite dimensional and

$$
P_{j} P_{j}=P_{i} P_{j}=\delta_{i j} P_{j}
$$

iii) The operator $H$ can be written as a norm convergence sum

$$
H=\sum_{j=1}^{\infty} \lambda_{j} P_{j} .
$$

iv) There is a complete orthonormal system $\left\{\psi_{j}\right\} \subset \mathscr{H}$ of eigenfunctions of $H$ such that for all $\phi \in H$ :

$$
H \phi=\sum_{j=1}^{\infty} \lambda_{j}\left\langle\phi, \psi_{j}\right\rangle \psi_{j},
$$

where $\psi_{j}$ is the eigenfunction corresponding to eigenvalue $\lambda_{j}$. 
Example A.44. i) A finite rank operator is compact.

ii) The operator $H: L^{2}(X, d x) \rightarrow L^{2}(Y, d y)$ defined by

$$
H f(y)=\int_{Y} K(x, y) f(x) d x,
$$

where $K(x, y) \in L^{2}(X \times Y, d x d y)$, is a compact operator.

Theorem A.45 ([RS80, Analytic Fredholm theorem]). Let D be an open connected subset of $\mathbb{C}$. Let $f: D \rightarrow \mathscr{L}(\mathscr{H})$ be an analytic operator-valued function such that $f(z)$ is compact for each $z \in D$. Then, either

i) $(I-f(z))^{-1}$ exists for no $z \in D$.

ii) $(I-f(z))^{-1}$ exists for all $z \in D \backslash S$ where $S$ is a discrete subset of $D$. In this case, $(I-f(z))^{-1}$ is meromorphic in $D$, analytic in $D \backslash S$, the residues at poles are finite rank operators, and if $z \in S$ then $f(z) \psi=\psi$ has a non-zero solution in $\mathscr{H}$.

Theorem A.46 ([HS96, Fredholm alternative]). Let $H$ be a compact operator on $\mathscr{H}$. Then:

i) The equation $\psi-H \psi=\phi$ has a unique solution for every $\phi \in \mathscr{H}$ if and only if $1 \notin \sigma(H)$.

ii) If $1 \in \sigma(H)$, then $\psi-H \psi=\phi$ has a unique solution if and only if $\phi \in\left[\operatorname{ker}\left(I-H^{*}\right)\right]^{\perp}$.

Definition A.47. Let $A$ be a closed operator with $\rho(A) \neq \varnothing$. An operator $B$ is called relatively $A$-compact if

i) $D(B) \supseteq D(A)$,

ii) $B R_{A}(\lambda)$ is compact for some (and hence for all) $\lambda \in \rho(A)$.

The next theorem shows that relative compactness is stronger than relative bounded. Theorem A.48 ([HS96]). Suppose $A$ is a self-adjoint operator and B is relatively Acompact. Then, B is A-bounded with relative bound zero.

The essential spectrum $\sigma_{\text {ess }}$ is stable under relatively compact perturbation.

Theorem A.49 ([HS96, Weyl's theorem]). Let A and B be self-adjoint operators, and $A-B$ be relatively $A$-compact. Then,

$$
\sigma_{\text {ess }}(A)=\sigma_{\text {ess }}(B) .
$$


One important class of potentials is Kato potential which is defined as follow:

Definition A.50. A potential $V$ is called a Kato potential if $V$ is real and $V \in L^{2}\left(\mathbb{R}^{d}\right)+$ $L^{\infty}\left(\mathbb{R}^{d}\right)_{\epsilon}$, where the $\epsilon$ indicates that for any $\epsilon>0, V$ can be written as a sum of $V_{1}+V_{2}$ with $V_{1} \in L^{2}\left(\mathbb{R}^{d}\right)$ and $V_{2} \in L^{\infty}\left(\mathbb{R}^{d}\right)$ with $\left\|V_{2}\right\|_{\infty}<\epsilon$.

Theorem A.51 ([HS96, Theorem 14.9]). If $V$ is real Kato potential, then $V$ is relatively $-\Delta$-compact. Consequently,

$$
\sigma_{\text {ess }}(-\Delta+V)=\sigma_{\text {ess }}(-\Delta)=[0, \infty)
$$

Remark A.52. The spectrum of Schrödinger operator $-\Delta+V(x)$ deeply depends on the behavior of the potential $V$. If $V$ is Kato potential, then spectrum of $-\Delta+V(x)$ consists of the half-line $[0, \infty)$ and negative discrete eigenvalues. If the potential $V$ tends to infinity, then the spectrum is purely discrete.

Theorem A.53 ([RS78, Theorem XIII.67]). Let $V \in L_{\mathrm{loc}}^{1}\left(\mathbb{R}^{d}\right)$ be bounded from below and suppose that $V(x) \rightarrow \infty$ as $|x| \rightarrow \infty$. Then, $H=-\Delta+V$ has compact resolvent. In particular, $H$ has purely discrete spectrum and a complete set of eigenfunctions.

\section{A.2 Analytic perturbation theory}

This section is to devote to recall some classical results on analytic perturbation theory (For more details, see [Rel69, Kat95, RS78]).

First, we recall the definition of bounded regular operators.

Definition A.54 ([Re169]). A family of linear bounded operators $H(\zeta)$ on a Hilbert space $\mathscr{H}$ is said to be regular in a real (complex) neighborhood of $\zeta=0$ if for each $f \in \mathscr{H}$, $H(\zeta) f$ is a power series in $\mathscr{H}$.

Theorem A.55 ([Re169]). A family of linear bounded operators $H(\zeta)$ on a Hilbert space $\mathscr{H}$ is regular near $\zeta=0$ if and only if there exists a sequence of bounded operators $H_{0}, H_{1}, \ldots$ independent of $\zeta$ and a positive number $c$ such that for each $f \in \mathscr{H}$,

1) $H(\zeta) f=H_{0} f+\zeta H_{1} f+\cdots$ is a convergent power series near $\zeta=0$,

2) $\left\|H_{n} f\right\| \leq c^{n+1}\|f\|, n=1,2, \ldots$. 
The definition of the bounded regular operators can be extended to the unbounded case.

Definition A.56 ([Re169]). A family of linear operators $H(\zeta)$ on a Hilbert space $\mathscr{H}$ with domains $D(\zeta)$ defined for each $\zeta$ in a real (complex) neighborhood of $\zeta=0$ is said to be regular with respect to $\zeta$ if the following two conditions are satisfied in a real (complex) neighborhood of $\zeta=0$ :

i) There exist a bounded regular operator $U(\zeta)$ mapping the whole Hilbert space $\mathscr{H}$ in a one-to-one way onto $D(\zeta)$.

ii) The element $H(\zeta) U(\zeta) f$ is a regular element for each $f \in \mathscr{H}$, that means there exists a power series with respect to $\zeta$.

Theorem A.57 ([Re169, Chapter 2, Section 5, Theorem 3]). Suppose that $H(\zeta)$ with the domain $D(\zeta)$ is Hermitian for real $\zeta$ and regular in a complex neighborhood of $\zeta=0$. Suppose that $H(0)$ on $D(0)$ is self-adjoint. Suppose that $\lambda$ is an eigenvalue of finite multiplicity $n$ of operator $H(0)$, and suppose there are positive numbers $h_{1}, h_{2}$ such that the spectrum of $H(0)$ in an open interval $\left(\lambda-h_{1}, \lambda+h_{2}\right)$ consists of exactly the point eigenvalue $\lambda$. Then, there exist power series of

$$
\lambda_{1}(\zeta), \lambda_{2}(\zeta), \ldots, \lambda_{n}(\zeta)
$$

and power series in the Hilbert space $\mathscr{H}$ of

$$
\psi_{1}(\zeta), \psi_{2}(\zeta), \ldots, \psi_{n}(\zeta)
$$

all convergent in a neighborhood of $\zeta=0$, which satisfy the following conditions:

i) The element $\psi_{j}(\zeta)$ is the eigenfunctions of $H(\zeta)$ on $D(\zeta)$ with respect to the eigenvalue $\lambda_{j}(\zeta)$. Furthermore $\lambda_{j}(0)=\lambda, j=1, \ldots, n$, and for real $\zeta$ the eigenfunctions form an orthonormal set.

ii) For each pair of positive numbers $h_{1}^{\prime}, h_{2}^{\prime}$ with $h_{1}^{\prime}<h_{1}$ and $h_{2}^{\prime}<h_{2}$, there exists a positive number $\rho$ such that the spectrum of $H(\zeta)$ on $D(\zeta)$ in $\left[\lambda_{1}(\zeta)-h_{1}^{\prime}, \lambda_{1}(\zeta)+h_{2}^{\prime}\right]$ for real $\zeta$ with $|\zeta|<\rho$ consists exactly of the points $\lambda_{1}(\zeta), \ldots, \lambda_{n}(\zeta)$.

We list here some criteria for regular family of operators: 
Theorem A.58 ([Re169]). A family of operators $H(\zeta)$ on a Hilbert space $\mathscr{H}$ with domains $D(\zeta)$ is regular if one of the following conditions holds

i) There exists a complex number $\gamma$ independent of $\zeta$ such that in a neighborhood of $\zeta=0$ the operators $H(\zeta)+\gamma$ has a regular and bounded inverse with domain $\mathscr{H}$.

ii) The operator $H(\zeta)$ on the domain $D$ is Hermitian in a real neighborhood of $\zeta=0$ and that $D$ is independent of $\zeta, H(0)$ is self-adjoint on $D$. And $H(\zeta) f$ is regular for each $f \in D$.

One question is that the set of eigenfunctions is complete or not. This leads to the following definition

Definition A.59 ([Rel69]). Let $H(\zeta)$ on $D(\zeta)$ be Hermitian operators for $h_{1}<\zeta<h_{2}$. We say that they have regular discrete spectrum in this interval if functions $\lambda_{1}(\zeta), \lambda_{2}(\zeta), \ldots$ and $\psi_{1}(\zeta), \psi_{2}(\zeta), \ldots$ on $D(\zeta)$ exist, all regular in a neighborhood of every $\zeta$ of this interval, such that for every $\zeta \in\left(h_{1}, h_{2}\right)$ the following statements hold

i) $H(\zeta) \psi_{n}(\zeta)=\lambda_{n}(\zeta) \psi_{n}(\zeta), \quad n=1,2, \ldots$,

ii) $\psi_{1}(\zeta), \psi_{2}(\zeta), \ldots$ is a complete orthonormal system,

iii) $\lim _{n \rightarrow \infty}\left|\lambda_{n}(\zeta)\right|=\infty$.

Theorem A.60 ([Re169, Chapter 2, Section 10, Theorem 1]). For real $\zeta$ near 0, let $H(\zeta)$ be a Hermitian operator with a domain $D$ which is independent of $\zeta$. Suppose that $H(\zeta) f$ is regular for every $f \in D$ and small $|\zeta|$. Suppose that $H(0)$ with the domain $D$ is self-adjoint and has the discrete spectrum. Then, $H(\zeta)$ has a regular discrete spectrum near $\zeta=0$.

Analytic perturbation theory was developed by Kato. Here, we recall some important results in [RS78, Kat95].

Definition A.61 ([RS78, Kat95]). A family of operators $H(\zeta)$ on a complex domain $D$ is called an analytic family in the sense of Kato if and only if

i) For each $\zeta \in D, H(\zeta)$ is closed and has a non-empty resolvent set.

ii) For every $\zeta^{*} \in D$, there is a $\lambda^{*} \in \rho\left(H\left(\zeta^{*}\right)\right)$ so that $\lambda^{*} \in \rho(H(\zeta))$ for $\zeta$ close to $\zeta^{*}$ and $\left(H(\zeta)-\lambda^{*}\right)^{-1}$ is an analytic operator-valued function for $\zeta$ near $\zeta^{*}$. 
Theorem A.62 ([RS78, Kato-Rellich theorem]). Let $H(\zeta)$ be an analytic family in the sense of Kato on the domain D. Let $\lambda^{*}$ be a non-degenerated (simple) discrete eigenvalue of $H\left(\zeta^{*}\right), \zeta^{*} \in D$. Then, for $\zeta$ near $\zeta^{*}$, there exists exactly one point $\lambda(\zeta)$ of $\sigma(H(\zeta))$ near $\zeta^{*}$ and this point is isolated and non-degenerated. Moreover, $\lambda(\zeta)$ is an analytic function of $\zeta$ near $\zeta^{*}$, and corresponding eigenvector $\psi(\zeta)$ can be chosen as an analytic function of $\zeta$ near $\zeta^{*}$. If $H(\zeta)$ is self-adjoint for $\zeta-\zeta^{*}$ real, then $\psi(\zeta)$ can be chosen to be normalized for $\zeta-\zeta^{*}$ real.

Theorem A.63 ([RS78, Theorem XII.13]). Let $H(\zeta)$ be an analytic family in the sense of Kato for $\zeta$ near 0 which is self-adjoint for $\zeta$ real. Let $\lambda^{*}$ be a discrete eigenvalue of multiplicity $m$ of $H(0)$. Then, there are $m$ (not necessarily distinct) single-valued functions, analytic near $\zeta=0, \lambda_{1}(\zeta), \lambda_{2}(\zeta), \ldots, \lambda_{m}(\zeta)$ with $\lambda_{k}(0)=\lambda^{*}$, so that $\lambda_{1}(\zeta), \lambda_{2}(\zeta), \ldots, \lambda_{m}(\zeta)$ are eigenvalues of $H(\zeta)$ for $\zeta$ near 0 . Further, these are the only eigenvalues near $\lambda^{*}$.

It is not convenient to check criteria in A.61. There is a simple criterion for analytic family of operators.

Definition A.64 ([Kat95]). Let $D$ be a connected domain in the complex plane and let $H(\zeta)$ be a closed operator with non-empty resolvent set. $H(\zeta)$ is said to be an analytic family of type (A) if and only if

i) The domain of $H(\zeta)$ is some set $\mathscr{K}$ independent of $\zeta \in D$.

ii) For each $\phi \in \mathscr{K}, H(\zeta) \phi$ is an analytic vector function of $\zeta$.

Theorem A.65 ([RS78]). Every analytic family of type (A) is an analytic family in the sense of Kato.

Theorem A.66 ([RS78, Theorem XII.9]). Let $H_{0}$ be a closed operator with non-empty resolvent set. Define $H_{0}+\zeta V$ on $D\left(H_{0}\right) \cap D(V)$. Then, $H_{0}+\zeta V$ is an analytic family of type (A) near $\zeta=0$ if and only if $V$ is $H_{0}$-bounded.

Theorem A.67 ([Kat95, Chapter 7, Theorem 2.4]). Let $H(\zeta)$ be a analytic family of operators of type (A). Then, $H(\zeta)$ has compact resolvent either for all $\zeta$ or for no $\zeta$.

Theorem A.68 ([Kat95, Chapter 7, Theorem 3.9]). Let $H(\zeta)$ be a self-adjoint analytic family of type (A) defined for $\zeta$ in a neighborhood of an interval $I_{0}$ of the real axis. Furthermore, assume $H(\zeta)$ has compact resolvent. Then, all eigenvalues of $H(\zeta)$ can 
be represented by functions which are analytic on $I_{0}$. More precisely, there is a sequence of repeated eigenvalues $\lambda_{j}(\zeta)$ and eigenfunctions $\psi_{j}(\zeta)$ of $H(\zeta)$, all analytic on $I_{0}$. Moreover, $\psi_{j}(\zeta)$ form a complete orthonormal basis.

Remark A.69. This theorem is a different form of Theorem A.60.

\section{A.3 The stationary phase method}

This section is to recall some classical results on the stationary phase method. We refer to [Coo82, Hör83, Won01, Zwo12] for more details.

Consider a oscillatory integral

$$
I(\lambda)=\int_{\mathbb{R}^{d}} e^{i \lambda f(x)} g(x) d x .
$$

Definition A.70. The function $f$ has a non-degenerated critical point at $x_{0}$ if

$$
f^{\prime}\left(x_{0}\right)=0, \quad \operatorname{det} f^{\prime \prime}\left(x_{0}\right) \neq 0 .
$$

One gets the rapid decay when there is no critical point.

Theorem A.71. Let $f \in C^{\infty}\left(\mathbb{R}^{d}\right)$ be real-valued, and $g \in C_{\mathrm{c}}^{\infty}\left(\mathbb{R}^{d}\right)$. If $f^{\prime} \neq 0$ on supp $g$, then for arbitrary $N>0$

$$
I(\lambda)=O\left(\lambda^{-N}\right) \quad \text { as } \lambda \rightarrow+\infty .
$$

Denote $\operatorname{sgn} f^{\prime \prime}\left(x_{0}\right)$ the number of positive eigenvalues of $f^{\prime \prime}\left(x_{0}\right)$ - the number of negative eigenvalues of $f^{\prime \prime}\left(x_{0}\right)$. The next theorem shows the asymptotic expansion of the oscillatory integral when there is only one non-degenerated critical point.

Theorem A.72 ( [Zwo12, Theorem 3.16], stationary phase asymptotics). Let $f \in C^{\infty}\left(\mathbb{R}^{d}\right.$ ) be real-valued, and $g \in C_{\mathrm{c}}^{\infty}\left(\mathbb{R}^{d}\right)$. Suppose $x_{0} \in K:=\operatorname{supp} f$ is a non-degenerated critical point, and $f^{\prime}(x) \neq 0$ on $K \backslash\left\{x_{0}\right\}$. Then,

i) There exist for each $k=0,1, \ldots$, differential operators $A_{2 k}(x, D)$ of order less than or equal to $2 k$ such that for all $N$

$$
\left|I(\lambda)-\left(\sum_{k=0}^{N-1} A_{2 k}(x, D) g\left(x_{0}\right) \lambda^{-\left(k+\frac{d}{2}\right)}\right) e^{i \lambda f\left(x_{0}\right)}\right| \leq C_{N} \lambda^{-\left(N+\frac{d}{2}\right)} \sum_{0 \leq m \leq 2 N+d+1} \sup \left|g^{(m)}\right|,
$$

where $C_{N}$ depends also on $K$. 
ii) In particular,

$$
A_{0}=(2 \pi)^{d / 2}\left|\operatorname{det} f^{\prime \prime}\left(x_{0}\right)\right|^{-1 / 2} e^{\frac{i \pi}{4} \operatorname{sgn} f^{\prime \prime}\left(x_{0}\right)},
$$

and consequently,

$$
I(\lambda)=\left(\frac{2 \pi}{\lambda}\right)^{d / 2}\left|\operatorname{det} f^{\prime \prime}\left(x_{0}\right)\right|^{-1 / 2} e^{\frac{i \pi}{4} \operatorname{sgn} f^{\prime \prime}\left(x_{0}\right)} e^{i \lambda f\left(x_{0}\right)} g\left(x_{0}\right)+O\left(\frac{1}{\lambda^{\frac{d+2}{2}}}\right)
$$

as $\lambda \rightarrow+\infty$.

The smoothness of $f, g$ in Theorem A.72 is a strong condition. It is necessary to derive the full asymptotic expansion. If only finite part of the asymptotic expansion is needed, then one gets the stationary phase method with less regularity.

Theorem A.73 ([Hör83, Theorem 7.7.5]). Let $K \subset \mathbb{R}^{d}$ be a compact set, $X$ an open neighborhood of $K$ and $k$ a positive integer. If $g(x) \in C_{\mathrm{c}}^{2 k}(K), f(x) \in C^{3 k+1}(X)$ and $\operatorname{Im} f \geq 0$ in $X, \operatorname{Im} f\left(x_{0}\right)=0, f^{\prime}\left(x_{0}\right)=0, \operatorname{det} f^{\prime \prime}\left(x_{0}\right) \neq 0, f^{\prime} \neq 0$ in $K \backslash\left\{x_{0}\right\}$ then

$$
\begin{aligned}
\left|\int_{K} g(x) e^{i \lambda f(x)} d x-e^{i \lambda f\left(x_{0}\right)}\left(\operatorname{det}\left(\lambda f^{\prime \prime}\left(x_{0}\right) / 2 \pi i\right)\right)^{-\frac{1}{2}} \sum_{j<k} \lambda^{-j} L_{j} g\right| & \\
& \leq C \lambda^{-k-d / 2} \sum_{|\alpha| \leq 2 k} \sup \left|D^{\alpha} g\right| .
\end{aligned}
$$

Here $C$ is bounded when $f$ stays in a bounded set in $C^{3 k+1}(X)$ and $\left|x-x_{0}\right| /\left|f^{\prime}(x)\right|$ has a uniform bound. With

$$
h_{x_{0}}(x)=g(x)-g\left(x_{0}\right)-\left\langle g^{\prime \prime}\left(x_{0}\right)\left(x-x_{0}\right), x-x_{0}\right\rangle / 2
$$

which vanishes of third order at $x_{0}$, we have

$$
L_{j} g=\sum_{v-\mu=j} \sum_{2 v \geq 3 \mu} i^{-j} 2^{-v}\left\langle f^{\prime \prime}\left(x_{0}\right)^{-1} D, D\right\rangle^{v}\left(h_{x_{0}}^{\mu} g\right)\left(x_{0}\right) /(\mu ! v !) .
$$

This is a differential operator of order $2 j$ acting on $g$ at $x_{0}$. The coefficients are rational homogeneous functions of degree $-j$ in $f^{\prime \prime}\left(x_{0}\right), \ldots, f^{2 j+2}\left(x_{0}\right)$ with denominator $\left(\operatorname{det} f^{\prime \prime}\left(x_{0}\right)\right)^{3 j}$. In every term the total number of derivatives of $g$ and $f^{\prime \prime}$ is at most $2 j$.

In particular, $L_{0}=I$ and consequently

$$
\int g(x) e^{i \lambda f} d x=e^{i \lambda f\left(x_{0}\right)}\left(\operatorname{det}\left(\lambda f^{\prime \prime}\left(x_{0}\right) / 2 \pi i\right)\right)^{-\frac{1}{2}}+O\left(\lambda^{-(d+2) / 2}\right) \quad \text { as } \quad \lambda \rightarrow+\infty .
$$




\section{Bibliography}

[BA11] M. Ben-Artzi. Smooth spectral calculus. In Partial differential equations and spectral theory, volume 211 of Oper. Theory Adv. Appl., pages 119-182. Birkhäuser/Springer Basel AG, Basel, 2011.

[BES13] M. Brown, M. Eastham, and M. Schmidt. Periodic differential operators, volume 230 of Operator Theory: Advances and Applications. Birkhäuser/Springer Basel AG, Basel, 2013.

[Bło92] Z. Błocki. Singular sets of separately analytic functions. Ann. Polon. Math., 56(2):219-225, 1992.

[Bor46] G. Borg. Eine Umkehrung der Sturm-Liouvilleschen Eigenwertaufgabe. Bestimmung der Differentialgleichung durch die Eigenwerte. Acta Math., 78:196, 1946.

[BY94] M. Sh. Birman and D. R. Yafaev. The scattering matrix for a perturbation of a periodic Schrödinger operator by decreasing potential. Algebra i Analiz, 6(3):17-39, 1994.

[Coo82] J. C. Cooke. Stationary phase in two dimensions. IMA J. Appl. Math., 29(1):25-37, 1982.

[Eas73] M. S. P. Eastham. The spectral theory of periodic differential equations. Texts in Mathematics (Edinburgh). Scottish Academic Press, Edinburgh; Hafner Press, New York, 1973.

[Ër̆d69] D. M. Èŭdus. The principle of limiting amplitude. Uspehi Mat. Nauk, 24(3(147)):91-156, 1969. 
[Eva10] L. C. Evans. Partial differential equations, volume 19 of Graduate Studies in Mathematics. American Mathematical Society, Providence, RI, Second edition, 2010.

[Fir75] N. E. Firsova. The Riemann surface of a quasimomentum, and scattering theory for a perturbed Hill operator. Zap. Naučn. Sem. Leningrad. Otdel. Mat. Inst. Steklov (LOMI), 51:183-196, 220, 1975.

[FJ16] S. Fliss and P. Joly. Solutions of the time-harmonic wave equation in periodic waveguides: asymptotic behaviour and radiation condition. Arch. Ration. Mech. Anal., 219(1):349-386, 2016.

[Fli09] S. Fliss. Etude mathématique et numérique de la propagation des ondes dans des milieux périodiques localement perturbés. $\mathrm{PhD}$ thesis, 2009.

[Fo195] G. B. Folland. Introduction to partial differential equations. Princeton University Press, Princeton, NJ, second edition, 1995.

[Gho02] M. Ghomi. Gauss map, topology, and convexity of hypersurfaces with nonvanishing curvature. Topology, 41(1):107-117, 2002.

[GT01] D. Gilbarg and N. S. Trudinger. Elliptic partial differential equations of second order. Classics in Mathematics. Springer-Verlag, Berlin, 2001.

[Hoa11] V. Hoang. The limiting absorption principle for a periodic semi-infinite waveguide. SIAM J. Appl. Math., 71(3):791-810, 2011.

[Hoa15] V. Hoang. A radiation condition for a periodic semi-infinite waveguide. Preprint, 2015.

[Hör83] L. Hörmander. The analysis of linear partial differential operators. I. Distribution theory and Fourier analysis, volume 256 of Grundlehren der Mathematischen Wissenschaften. Springer-Verlag, Berlin, 1983.

[Hör90] L. Hörmander. An introduction to complex analysis in several variables, volume 7 of North-Holland Mathematical Library. North-Holland Publishing Co., Amsterdam, Third edition, 1990. 
[HS96] P. D. Hislop and I. M. Sigal. Introduction to spectral theory: With applications to Schrödinger operators, volume 113 of Applied Mathematical Sciences. Springer-Verlag, New York, 1996.

[Kat95] T. Kato. Perturbation theory for linear operators. Classics in Mathematics. Springer-Verlag, Berlin, 1995.

[KKR17] M. Kha, P. Kuchment, and A. Raich. Green's function asymptotics near the internal edges of spectra of periodic elliptic operators: Spectral gap interior. to appear in J. Spectral Theory, 2017.

[Koh59] W. Kohn. Analytic properties of Bloch waves and Wannier functions. Phys. Rev. (2), 115:809-821, 1959.

[KR00] F. Klopp and J. Ralston. Endpoints of the spectrum of periodic operators are generically simple. Methods Appl. Anal., 7(3):459-463, 2000.

[KR12] P. Kuchment and A. Raich. Green's function asymptotics near the internal edges of spectra of periodic elliptic operators. Spectral edge case. Math. Nachr., 285(14-15):1880-1894, 2012.

[KS87] W. Kirsch and B. Simon. Comparison theorems for the gap of Schrödinger operators. Journal of Functional Analysis, 75(2):396 - 410, 1987.

[KT90] H. Knörrer and E. Trubowitz. A directional compactification of the complex Bloch variety. Comment. Math. Helv., 65(1):114-149, 1990.

[Kuc93] P. Kuchment. Floquet theory for partial differential equations, volume 60 of Operator Theory: Advances and Applications. Birkhäuser Verlag, Basel, 1993.

[Kuc16] P. Kuchment. An overview of periodic elliptic operators. Bull. Amer. Math. Soc. (N.S.), 53(3):343-414, 2016.

[MT03] M. Murata and T. Tsuchida. Asymptotics of Green functions and Martin boundaries for elliptic operators with periodic coefficients. J. Differential Equations, 195(1):82-118, 2003. 
[MT06] M. Murata and T. Tsuchida. Asymptotics of Green functions and the limiting absorption principle for elliptic operators with periodic coefficients. J. Math. Kyoto Univ., 46(4):713-754, 2006.

[MW66] W. Magnus and S. Winkler. Hill's equation. Interscience Tracts in Pure and Applied Mathematics, No. 20. Interscience Publishers John Wiley \& Sons New York-London-Sydney, 1966.

[Par08] L. Parnovski. Bethe-Sommerfeld conjecture. Ann. Henri Poincaré, 9(3):457508, 2008.

[Plu11] M. Plum. On the spectral of periodic differential operators. In Photonic crystals: Mathematical analysis and numerical approximation, volume 42 of Oberwolfach Seminars, pages 63-77. Birkhäuser/Springer Basel AG, Basel, 2011.

[PS10] L. Parnovski and A. Sobolev. Bethe-Sommerfeld conjecture for periodic operators with strong perturbations. Invent. Math., 181(3):467-540, 2010.

[Rad10] M. Radosz. The principles of limit absorption and limit amplitude for periodic operators. PhD thesis, Karlsruher Instituts für Technologie (KIT), 2010.

[Rad15] M. Radosz. New limiting absorption and limit amplitude principles for periodic operators. Z. Angew. Math. Phys., 66(2):253-275, 2015.

[Rel43] F. Rellich. Über das asymptotische Verhalten der Lösungen von $-\Delta u+\lambda u=$ 0 in unendlichen Gebieten. Jahresbericht der Deutschen MathematikerVereinigung, 53:57-65, 1943.

[Re169] F. Rellich. Perturbation Theory of Eigenvalue Problems. Notes on mathematics and its applications. Gordon and Breach, 1969.

[RS75] M. Reed and B. Simon. Methods of modern mathematical physics. II. Fourier analysis, self-adjointness. Academic Press, New York-London, 1975.

[RS78] M. Reed and B. Simon. Methods of modern mathematical physics. IV. Analysis of operators. Academic Press, New York-London, 1978. 
[RS80] M. Reed and B. Simon. Methods of modern mathematical physics. I. Functional analysis. Academic Press, New York, Second edition, 1980.

[Sch92] S. H. Schot. Eighty years of Sommerfeld's radiation condition. Historia Math., 19(4):385-401, 1992.

[Sic90] J. Siciak. Singular sets of separately analytic functions. Colloq. Math., 60/61(1):281-290, 1990.

[Sim76] B. Simon. On the genericity of nonvanishing instability intervals in Hill's equation. Ann. Inst. H. Poincaré Sect. A (N.S.), 24(1):91-93, 1976.

[Som10] A. Sommerfeld. Die Greensche Funktion der Schwingungsgleichung für ein beliebiges Gebiet. Phys. Z., 11:1057-1066, 1910.

[Som12] A. Sommerfeld. Die Greensche Funktion der Schwingungsgleichung. Jahresber. Dtsch. Math.-Ver., 21:309-353, 1912.

[SR90] J. Saint-Raymond. Fonctions séparément analytiques. Ann. Inst. Fourier (Grenoble), 40(1):79-101, 1990.

[Tay11] M. E. Taylor. Partial differential equations II. Qualitative studies of linear equations, volume 116 of Applied Mathematical Sciences. Springer, New York, Second edition, 2011.

[Tho73] L. E. Thomas. Time dependent approach to scattering from impurities in a crystal. Comm. Math. Phys., 33:335-343, 1973.

[Tit58] E. C. Titchmarsh. Eigenfunction expansions associated with second-order differential equations. Vol. 2. Oxford, at the Clarendon Press, 1958.

[Tit62] E. C. Titchmarsh. Eigenfunction expansions associated with second-order differential equations. Vol. 1. Second Edition. Clarendon Press, Oxford, 1962.

[Van97] N. T. Van. Separate analyticity and related subiects. Vietnam Journal of Mathematics, 25(2):81-90, 1997.

[Wil78] C. H. Wilcox. Theory of Bloch waves. J. Analyse Math., 33:146-167, 1978. 
[Won01] R. Wong. Asymptotic approximations of integrals, volume 34 of Classics in Applied Mathematics. Society for Industrial and Applied Mathematics (SIAM), Philadelphia, PA, 2001.

[Zwo12] M. Zworski. Semiclassical analysis, volume 138 of Graduate Studies in Mathematics. American Mathematical Society, Providence, RI, 2012. 